\title{
FREEZE-FRACTURE STUDIES ON BARLEY PLASTID MEMBRANES. III. LOCATION OF THE LIGHT-HARVESTING CHLOROPHYLL-PROTEIN
}

\author{
by \\ DAVID J. SIMPSON \\ Department of Physiology, Carlsberg Laboratory, \\ Gamle Carlsberg Vej 10, DK-2500 Copenhagen Valby
}

Keywords: Chloroplast, chlorina-f2, electron microscopy, light-harvesting complex, liposomes, mutant, particle size and shape, rotary shadowing

The thylakoids of chloroplasts from wild-type barley and the nuclear gene mutant chlorina $-f 2$ were examined by freeze-fracturing and rotary shadowing. The advantages of rotary shadowing were: the shape of freeze-fracture particles was revealed, particles on membrane surfaces were seen in greater detail, estimation of particle density was more reliable, and measurements could be made of membrane thickness. When wild-type and chlorina- $f 2$ thylakoid ultrastructure were compared, the most significant difference was the large reduction in the number of particles on the PFs face of the mutant. In addition. EFs particles were about $12 \%$ smaller in the mutant, whereas the appearance of the EFu, PFu and PSu was substantially the same as for wild-type thylakoids. These ultrastructural differences are correlated with the complete absence of the light-harvesting chlorophyll $a / b$. protein from chlorina $-f 2$ thylakoids. Freeze-fracturing of purified light-harvesting complex in vesicles revealed particles similar in size and shape to those missing from the PFs face of chlorina- $f 2$ but present on the PFs face of wild-type. It is concluded that in wild-type granal thylakoids, the light-harvesting chlorophyll $a / b$-protein is located in particles which are closely associated with EFs particles, but which cleave with the PFs face during freeze-fracturing.

\footnotetext{
Abbreviations: $\mathrm{EFs}=$ endoplasmic fracture (stacked), $\mathrm{EFu}=$ endoplasmic fracture (unstacked), $\mathrm{ES}=$ endoplasmic surface, ESs = endoplasmic surface (stacked), ESu = endoplasmic surface (unstacked), PFs = protoplasmic fracture (stacked), $\mathrm{PFu}=$ protoplasmic fracture (unstacked), $\mathrm{PSu}=$ protoplasmic surface (unstacked), S.D. = standard deviation, S.E. = standard error of the mean, Tricine $=\mathrm{N}$-(tris-(hydroxymethyl)methyl)glycine.
} 


\section{INTRODUCTION}

The structure of the chloroplast thylakoid membrane has been examined by a variety of physical and chemical techniques. These include $X$-ray diffraction, the use of chemical and fluorescent probes, spin-labels, circular dichroism and other spectroscopic methods, antibody labelling and electron microscopy. One of the powerful electron microscopic techniques is freeze-fracturing which, due to its unique ability to cleave the lipid bilayer along the internal hydrophobic plane, reveals structures within the membrane at a supramolecular level. The membrane surface can be studied by freezeetching, which enables the visualisation of structures on the membrane without chemical fixation or the damage caused by surface tension forces generated by air drying of material examined by negative staining.

When thylakoid membranes are freeze-fractured and examined with the electron microscope, many small particles are seen in the cleaved membrane. Evidence from reconstitution $(15,27,41)$ and specific labelling experiments (32) supports the idea that many freeze-fracture particles are composed of protein. Such particles are too large to consist of a single polypeptide, so it is probable that freeze-fracture particles consist of several polypeptides (14). There is an increasing amount of evidence in favour of the concept that the structural units visualised by freezefracturing consist of polypeptides organised into discrete functional units. This concept has been incorporated into a recent model of the thylakoid membrane $(4,40)$.

Ultimately, one would like to determine the localisation of all the polypeptide components of thylakoids and assign them to the various freezefracture particles found on the different fracture faces. One approach to this problem is to isolate structural and/or functional components of thylakoids. These can then be inserted into artificial lipid bilayer vesicles and their freezefracture ultrastructure examined $(4,41)$. In this way, the appearance of specific membrane polypeptide components can be studied in a relatively simple model system. The opposite, and complementary technique is to investigate the freeze-fracture ultrastructure of thylakoids from mutants which specifically lack one or more components $(36,37,45)$. Since there is an extensive and genetically well-characterised collection of barley plastid mutants (44), this species is an obvious choice for such an investigation.

The nuclear gene mutant of barley known as chlorina- $f 2$ lacks the light-harvesting chlorophyll $a / b$ protein $2\left(\mathrm{Ch}_{\mathrm{a} / \mathrm{b}}-\mathrm{P} 2\right)(25)$ and a small number of minor polypeptides $(2,10,17,24)$. $\mathrm{ChI}_{\mathrm{a} / \mathrm{h}}-\mathrm{P} 2$ is a major component of wild-type thylakoids and is a hydrophobic polypeptide (42), so it would be expected that there would be sufficient mass of this polypeptide located at the freeze-fracture plane for its absence to be detected. There is circumstantial evidence which suggests that $\mathrm{Chl}_{\mathrm{a} / \mathrm{b}}-\mathrm{P} 2$ is a component of the EF particles of grana $(3,39,40)$. Thus, freezefracture studies of chlorina- $f 2$ thylakoids have tended to concentrate on the size of these EFs particles, and the results are conflicting $(1,5,17$, $18,30)$. The most careful study indicates a slight reduction in EFs particle size of the mutant compared wild-type (30). This result is difficult to reconcile with the much larger change in EFs particle size reported during the greening of peas under conditions where one of the major polypeptides synthesised is $\mathrm{Chl}_{a / b}-\mathrm{P} 2$ (3).

In order to resolve this apparent conflict, the freeze-fracture ultrastructure of all four fracture faces and both surfaces of chlorina- 2 thylakoids was examined. These were compared with wildtype thylakoids isolated from plants of the same age and grown under the same conditions as the mutant (35). The isolation medium contained a higher concentration of divalent cations $\left(\mathrm{Mg}^{++}\right)$ to maintain stacking in the mutant $(10,41)$. In order to detect small changes in particle size, shape or density, the technique of rotary shadowing $(7,13,26,43)$ was used and the results are compared with those from conventional uni-directional shadowing.

\section{MATERIALS AND METHODS}

\subsection{Plant material}

Seeds of wild-type (Hordeum vulgare cv. Svalöfs Bonus) and mutant (chlorina-f2) barley were germinated in plastic trays containing vermiculite moistened with tap water. Seedlings were harvested after growing for 7 days at $20^{\circ} \mathrm{C}$ in continuous white light ( 1,700 lux $)$ as described previously (35). 


\subsection{Isolation of chloroplasts}

Precooled leaves were homogenised in 3 volumes of grinding medium at $4{ }^{\circ} \mathrm{C}$ using a kitchen blender with replaceable razor blades (20). The grinding medium consisted of $0.4 \mathrm{M}$ sucrose, 50mm-Tricine/ $\mathrm{NaOH}, \mathrm{pH} 7.9,5 \mathrm{~mm}-$ $\mathrm{MgCl}_{2}$ for wild-type and $0.4 \mathrm{M}$-sucrose, $50 \mathrm{~mm}$ Tricine $/ \mathrm{NaOH}, \quad \mathrm{pH} 7.9,25 \mathrm{~mm}-\mathrm{MgCl}_{2}$ for chlorina- $f 2$. The resulting slurry was filtered through 2 layers of $31 \mu \mathrm{m}$ nylon gauze and centrifuged for $5 \mathrm{~min}$ at $1,400 \mathrm{~g}$. The pellet was resuspended in a small volume of the appropriate grinding medium and an equal volume of $60 \%$ glycerol in 5 or $25 \mathrm{~mm}-\mathrm{MgCl}_{2}$ was added, followed by centrifugation for $5 \mathrm{~min}$ at $5,000 \mathrm{~g}$. This pellet was used for freeze-fracturing. Preparation of samples for freeze-etching involved washing the initial chloroplast pellet 3 times in $5 \mathrm{~mm}$-Tricine/ $\mathrm{NaOH}$, pH $7.95 \mathrm{~mm}-\mathrm{MgCl}_{2}$ (wild-type), or $10 \mathrm{~mm}-\mathrm{MgCl}_{2}$ (chlorina- 2 ), before a final centrifugation for $10 \mathrm{~min}$ at $10,000 \mathrm{~g}$.

\subsection{Freeze-fracturing}

Unfixed chloroplasts were frozen on gold planchettes in melting Freon 22 cooled by liquid nitrogen. Replicas were made with a Balzers BAF 301 freeze-etching apparatus (Balzers AG, Liechtenstein) equipped with a rotating cold stage that could be used for the preparation of both uni-directional and rotary shadowed replicas. Freeze-fracturing was performed at $-110^{\circ} \mathrm{C}$ and freeze-etching at $-100{ }^{\circ} \mathrm{C}$ for $2 \mathrm{~min}$ at a vacuum of $2 \times 10^{-6}$ torr or better. Subsequent treatment was as previously described (34). All uni-directionally shadowed micrographs are printed so that the direction of shadowing is from bottom to top, and the terminology used is that of Branton et al. (8).

\subsection{Calculations for rotary shadowing}

When freeze-fractured specimens are replicated with $\mathrm{Pt} / \mathrm{C}$ by uni-directional shadowing, it is usual to deposit a metal film to a depth of $20 \AA$. The deposition process can be monitored by the change in the frequency of oscillation of a quartz crystal. The arrangement of the electron beam gun, specimen stage and quartz crystal monitor is shown in Figure 1. The change in frequency is related to the metal film thickness by the formula:

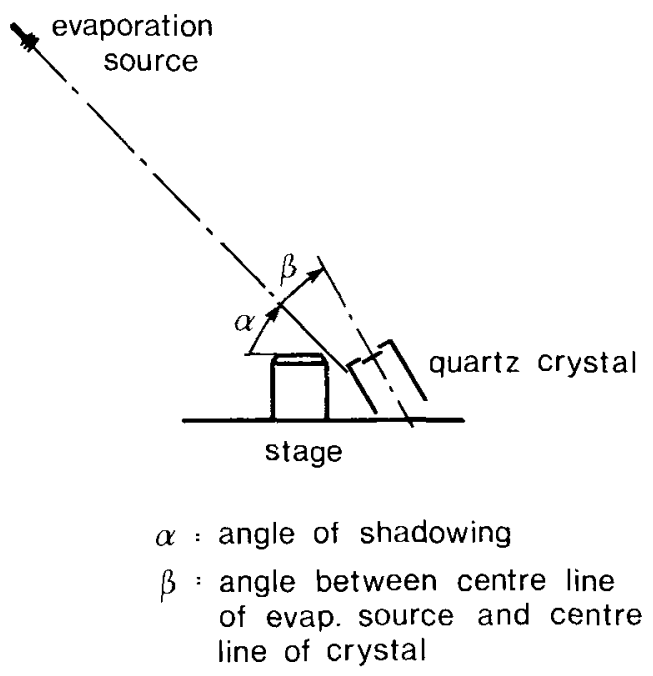

Figure 1. Diagram of the arrangement of the evaporation source, specimen and quartz crystal holder in the Balzers BAF301 freeze-etch unit.

$$
\Delta \mathrm{f}=\frac{\mathrm{k} \cdot \mathrm{d}_{1} \cdot \cos \beta}{\sin \alpha}
$$

Where $\Delta \mathrm{f}=$ frequency change $(\mathrm{Hz}) ; \mathrm{k}=$ material constant $(\mathrm{Hz} / \AA) ; \mathrm{d}_{1}=$ desired film thickness $(\AA) ; \alpha=$ angle of shadowing; $\beta=$ angle between centre line of evap. source and centre line of crystal. Usually, for uni-directional shadowing, $\alpha=45^{\circ}$ and $\beta=30^{\circ}$, and $a$ frequency change of $170 \mathrm{~Hz}$ corresponds to a film thickness of $20 \AA$ of $\mathrm{Pt} / \mathrm{C}$. If the angle of the quartz crystal holder is not altered, then $\beta=75^{\circ}$ $-\alpha$. If the shadowing angle $\alpha$ is changed to $\alpha$, then the frequency change $\left(\Delta \mathrm{f}^{\prime}\right)$ needed to produce a $20 \AA$ thick film is given by:

$$
\begin{aligned}
\Delta f^{\prime} & =\frac{\Delta f \cdot \cos \left(75-\alpha^{\prime}\right) \cdot \sin 45^{\circ}}{\sin \alpha^{\prime} \cdot \cos 30^{\circ}} \\
& =\frac{138.8 \cdot \cos \left(75-\alpha^{\prime}\right)}{\sin \alpha^{\prime}}
\end{aligned}
$$

Determination of values of $\Delta \mathrm{f}^{\prime}$ for different values of $\alpha$ are given in Table I. In practice it is not useful to use angles below $20^{\circ}$ because of shading of the quartz crystal by the coldstage, unless the angle of mounting of the crystal holder is altered. In this study, a shadowing angle of $22.5^{\circ}$ was used, with a corresponding $\Delta$ f'of $220 \mathrm{~Hz}$. 
Table I

Relationship between shadowing angle $(\alpha)$ and frequency change $\left(\Delta f^{\prime}\right)$ for deposition of a $20 \AA$ thick $\mathrm{Pt} / \mathrm{C}$ film.

\begin{tabular}{cc}
\hline Shadowing Angle $(\alpha)$ & $\Delta \mathrm{f}^{\prime}(\mathrm{Hz})$ \\
\hline $6^{\circ}$ & 476 \\
$10^{\circ}$ & 338 \\
$15^{\circ}$ & 268 \\
$20^{\circ}$ & 233 \\
$22.5^{\circ}$ & 221 \\
$25^{\circ}$ & 211 \\
$30^{\circ}$ & 196 \\
$45^{\circ}$ & 170 \\
\hline
\end{tabular}

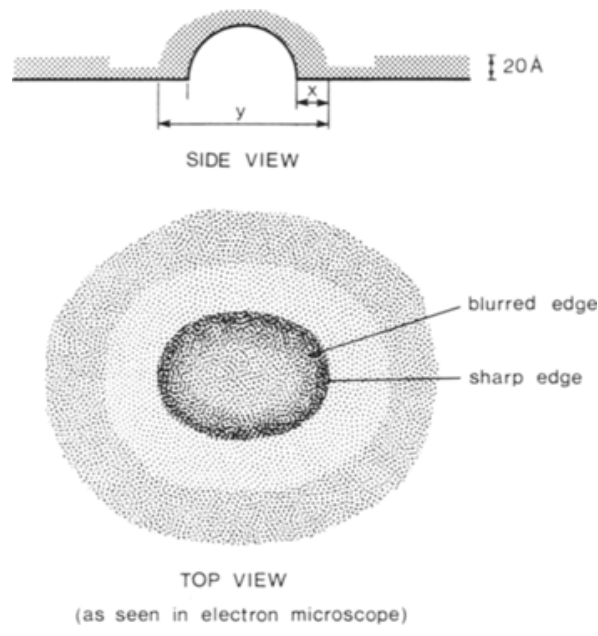

Figure 2. Predicted appearance of a hemispherical particle after rotary shadowing.
Because the specimen is rotating during shadowing, $\mathrm{Pt} / \mathrm{C}$ will be deposited on all sides of projecting freeze-fracture particles. The amount deposited on a hemisphere decreases from the base towards its top as the angle between the evaporation source and the particle surface decreases. Thus, as illustrated in Figure 2, a hemispherical particle after rotary shadowing is expected to appear as a dark ring surrounding a less dense central portion. The particle casts an annular shadow with a radius equal to the particle height divided by the sine of the shadowing angle (Figure 3). The thickness of the $\mathrm{Pt} / \mathrm{C}$ film in this region should therefore be less than $20 \AA$. If the particle does not have vertical sides, there will be no sharp edge corresponding to the position of the boundary between the particle and the lipid layer (Figure 2). To determine the true particle diameter, it is necessary to calculate the width of the film at the base of the particle. In this case the true value (D) is related to the measured value by the equation:

$$
\begin{aligned}
D=y-2 x \quad \text { where } y= & \text { measured diameter } \\
x= & \text { film thickness at } \\
& \text { base (calculated) }
\end{aligned}
$$

The value of ' $x$ ' can be calculated from a consideration of the amount of $\mathrm{Pt} / \mathrm{C}$ deposited at the base of a particle during shadowing. For a uni-directionally shadowed particle, the film thickness on a horizontal surface, measured in the direction of shadowing is given by:

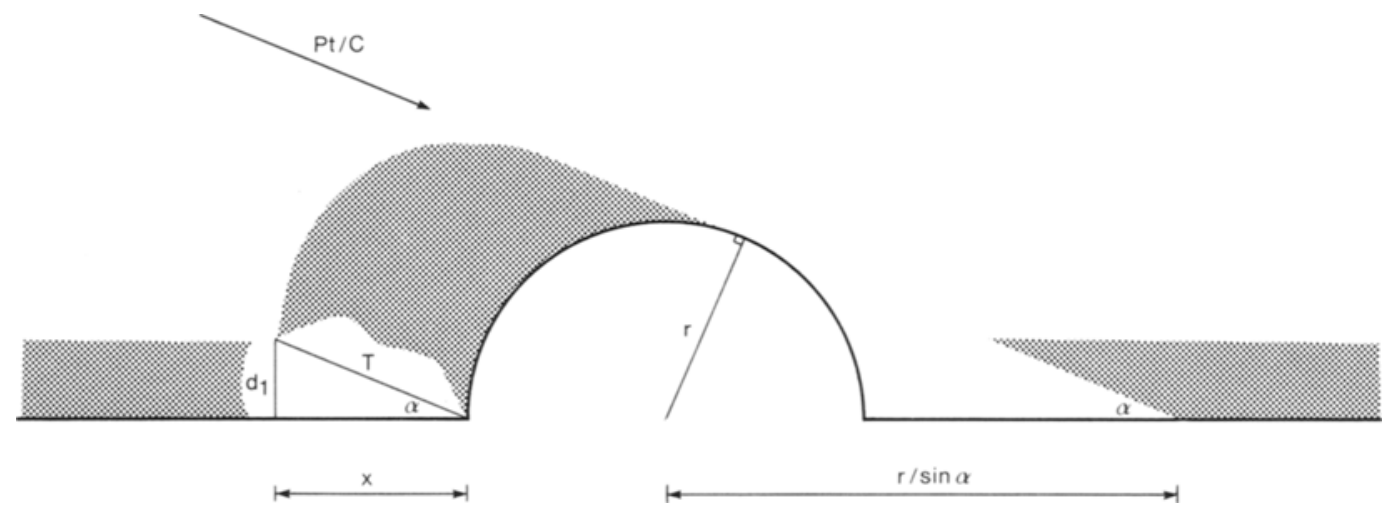

Figure 3. Diagram showing parameters used to calculate the width $(x)$ of the platinum layer at the base of a unidirectionally shadowed hemispherica! particle of height ' $r$ ', shadowing angle $\alpha$ and $d_{1}=$ thickness of platinum layer. 


$$
T=\frac{d_{1}}{\sin \alpha}
$$

where $d_{1}=$ film thickness determined by quartz crystal monitor

During rotary shadowing, however, while the value of $T$ is the same for flat areas as for unidirectional shadowing, the amount of $\mathrm{Pt} / \mathrm{C}$ deposited at the base of a particle is less since the same amount of $\mathrm{Pt} / \mathrm{C}$ must be evenly distributed over a larger area. The ratio of $\mathrm{Pt} / \mathrm{C}$ deposited at the base of a hemispherical particle during rotary shadowing compared with uni-directional shadowing for the same length of time is given by:

$$
\frac{\int_{0}^{\pi} K \sin \Theta \cdot d \Theta}{\int_{0}^{2 \pi} K \cdot d \Theta}
$$

where $\mathrm{K}=$ rate of $\mathrm{Pt} / \mathrm{C}$ deposition

This formula yields:

$$
\frac{[-\cos \Theta]_{0}^{\pi}}{[\Theta]_{0}^{2 \pi}}=1 / \pi
$$

That is, a rotary shadowed particle has a film thickness ( $T$ ) measured in the direction of shadowing, which is $1 / \pi$ of a particle shadowed without rotation. It follows from Figure 3 that the thickness of $\mathrm{Pt} / \mathrm{C}$ at the base of the particle $(\mathrm{x})$ is given by:

$$
\begin{aligned}
x & =\frac{T \cdot \cos \alpha}{\pi} \\
& =\frac{d_{1}}{\pi \cdot \tan \alpha}
\end{aligned}
$$

For $d_{1}=20 \AA$ and $\alpha=22.5^{\circ}, x=15.4 \AA$. For a uni-directionally shadowed particle with $\mathrm{d}_{1}=$ $20 \AA$ and $\alpha=45^{\circ}, \mathrm{x}=20 \AA$.

This means that a rotary shadowed particle with a measured diameter of $100 \AA$ has a true diameter of $100-2 \times 15.4=69 \AA$, i.e., its diameter would be overestimated by $45 \%$.

\subsection{Freeze-fracture particle measurements}

Particle densities (number of particles per square micron) were measured on micrographs enlarged to $200,000 \times$ (EF faces) or $400,000 \times$
(PF faces) as previously described (34). Particle size and size distribution were determined with a digitiser-computer-plotter system (34) from micrographs taken at $100,00 \times$ and enlarged to 800,000 . For rotary shadowed preparations, the long and short axis (measured at right angles) of each particle were recorded, and plotted separately, and the semi-axis ratio calculated. No correction was made for the thickness of the $\mathrm{Pt} / \mathrm{C}$ film.

\section{RESULTS}

Thylakoids of wild-type barley chloroplasts (Figure 4) are organised into stacked regions (grana) and unstacked regions. This is also true for the chloroplasts of chlorina-f2 (Figure 5). It was found that the wild-type grana had an average diameter of $0.42 \pm 0.05 \mu \mathrm{m}( \pm$ S.D.) and there were $10.4 \pm 4.8$ ( \pm S.D.) thylakoid discs per granum. This can be compared with chlorina- $f 2$ chloroplasts in which the grana had an average diameter of $1.01 \pm 0.29 \mu \mathrm{m}( \pm$ S.D.) with $8.5 \pm 3.4$ ( \pm S.D.) thylakoid discs per granum (see Table VII).

The ultrastructure of rotary shadowed freezefractured thylakoids from wild-type barley is shown in Figure 6. Although the visual appearance of such a preparation differed considerably from uni-directionally shadowed thylakoids, the four different fracture faces could be identified on the basis of differences in particle size and density (see 35). They are shown at higher magnification in Figures 7-9. The particles on the EFs face (Figure 7) had the same appearance as shown diagrammatically in Figure 2. Each particle was bounded by a sharp edge which distinguished it from the lipid matrix. The centre of the particle was less electron dense, but there was not a clear boundary between this region and the platinum deposited at the base of the particle (see Figure 2). Visual inspection revealed that EFs particles varied not only in shape (from circular to elliptical), but also in size. The complementary PFs face (Figure 8) was densely covered with close-packed small particles. Most of these particles appeared to be uniformly sized but there were some particles that were slightly larger and more electron dense than the rest, as if they projected above the others. 


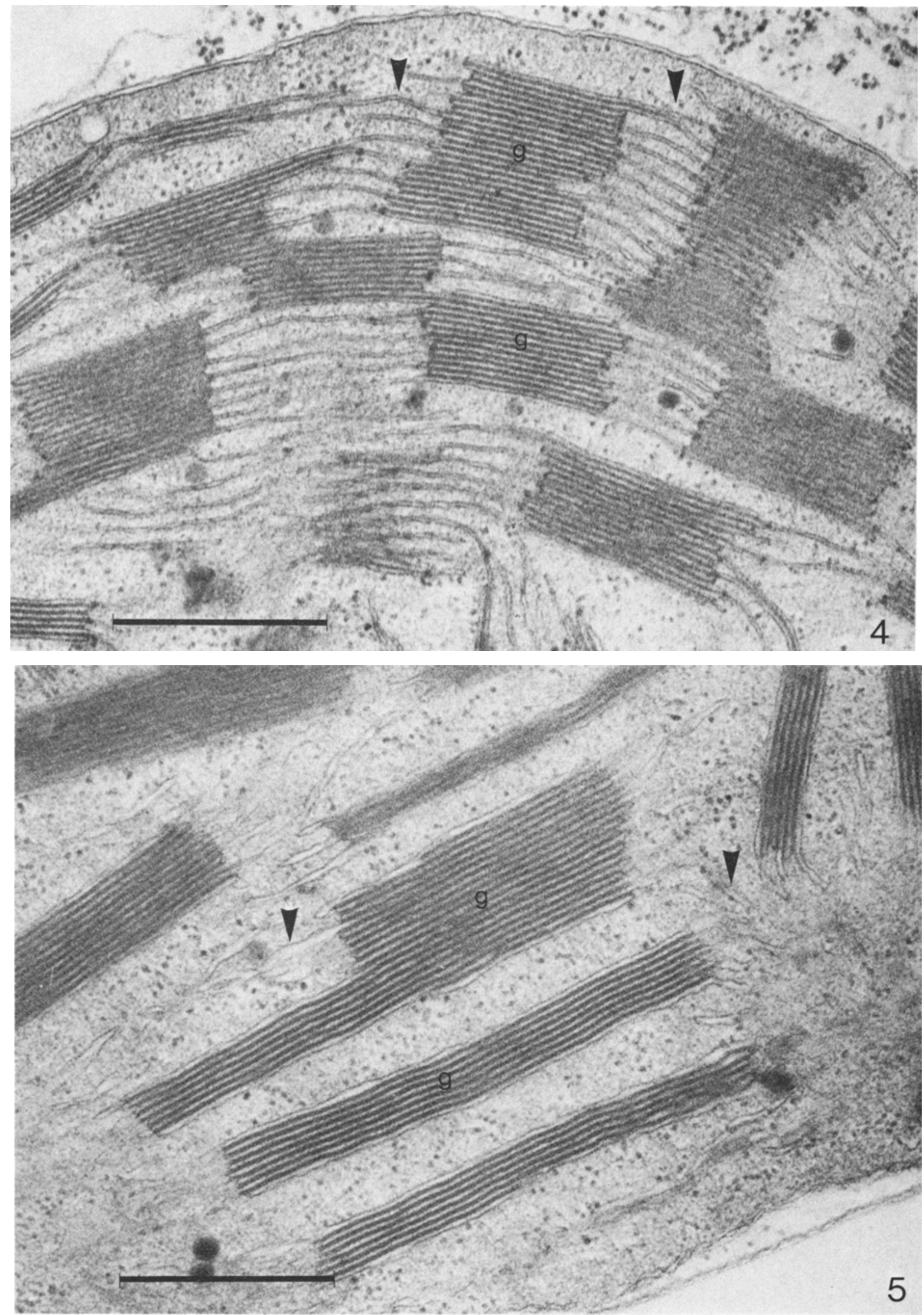




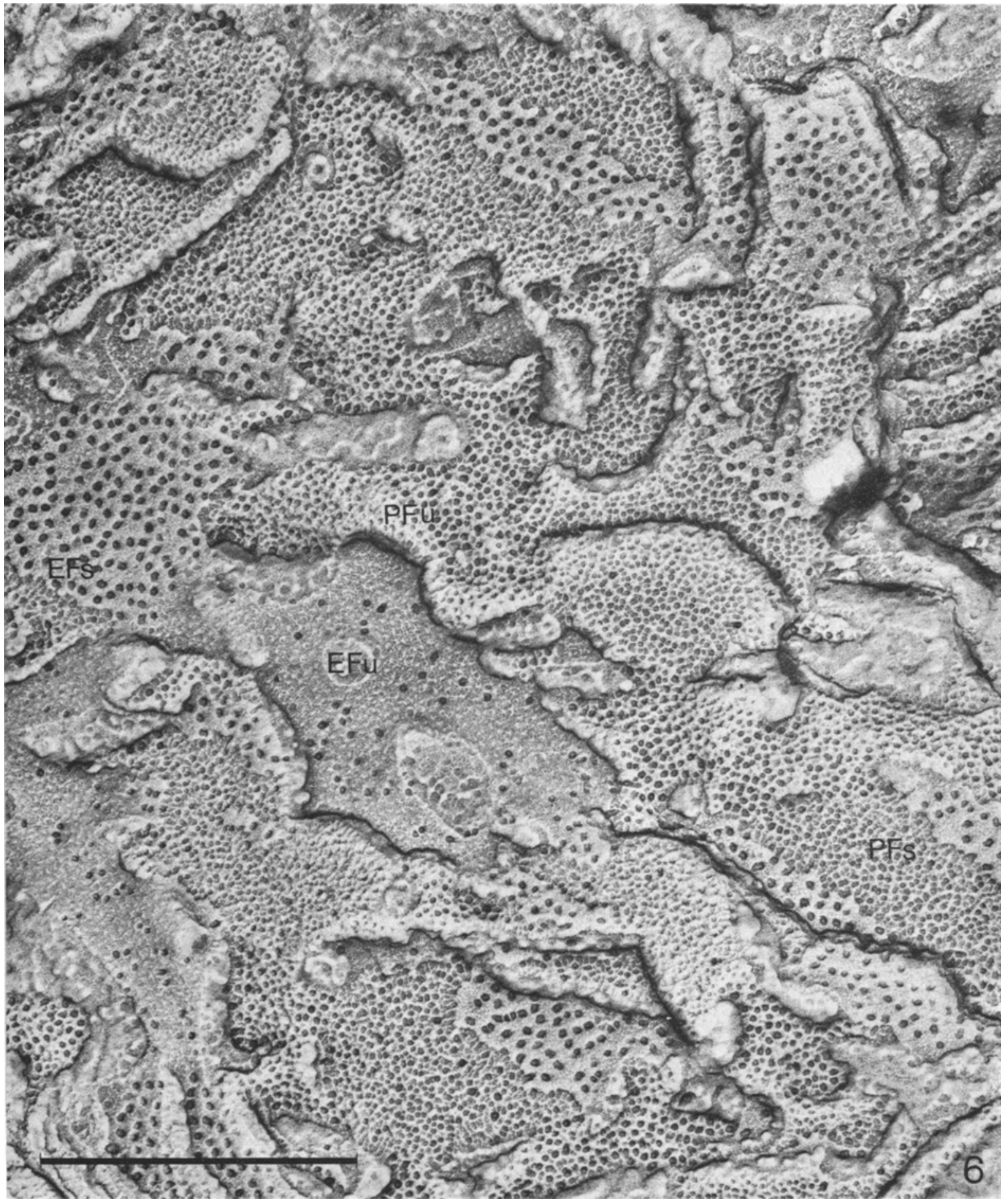

Figure 4. Thin-sectioned chloroplast from wild-type barley seedling leaf grown for seven days in continuous light.

Thylakoid membranes are differentiated into stacked regions called grana $(\mathrm{g})$ and unstacked regions (arrowheads). $\times 75,000($ Bar $=0.5 \mu \mathrm{m})$.

Figure 5. Thin-section of a chloroplast from chlorina- $f 2$ mutant of barley, grown under the same conditions as the wild-type in Figure 4.

Well-developed grana are clearly present at this stage $\times 75,000(\mathrm{Bar}=0.5 \mu \mathrm{m})$.

Figure 6. Rotary shadowed freeze-fractured thylakoids from wild-type barley chloroplasts showing the relationship between the four different fracture faces.

Particles are visible as electron dense deposits which cast no shadow, in contrast to particles in unidirectionally shadowed replicas. $\times 100,000(\mathrm{Bar}=0.5 \mu \mathrm{m})$. 


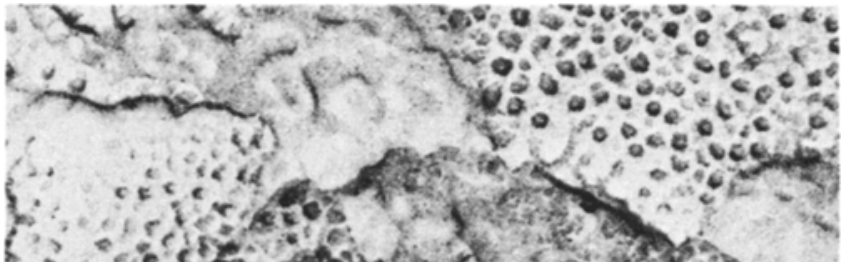
$33^{4}=87^{5}$.

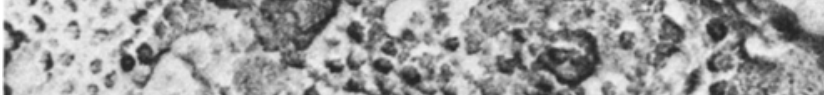

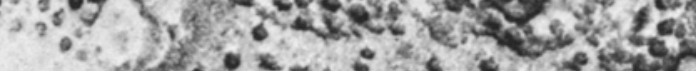

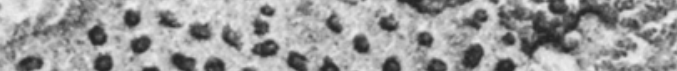

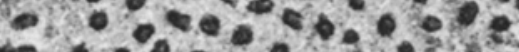

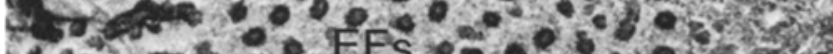

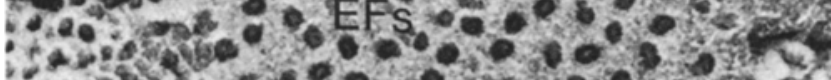

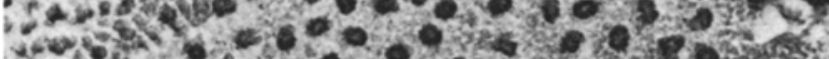

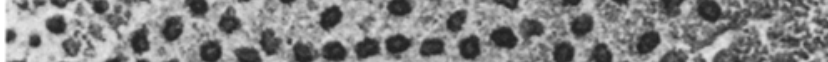

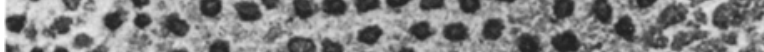

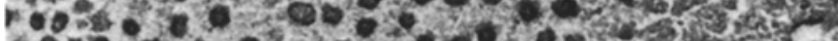

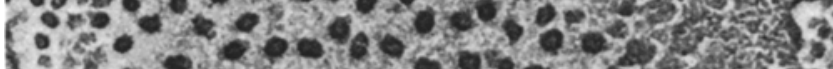

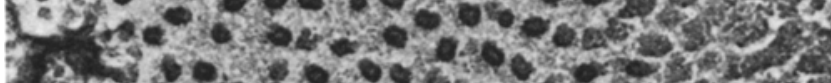
P. A.

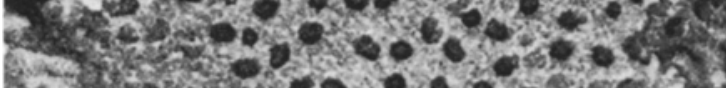

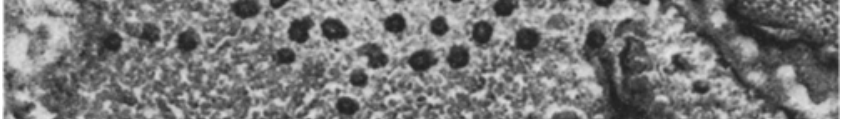

E.

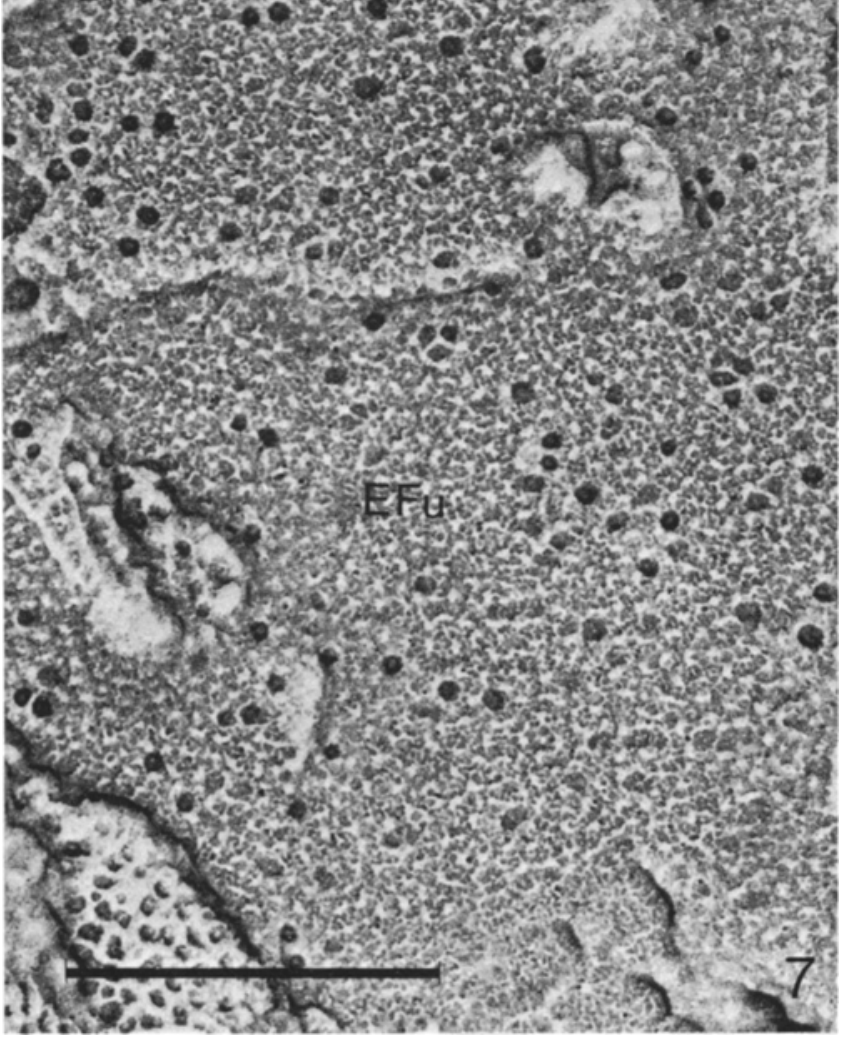

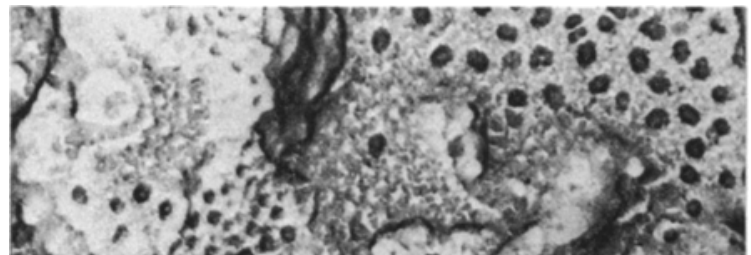
Prow

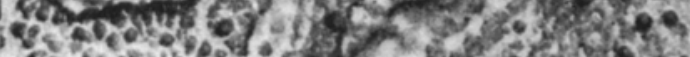

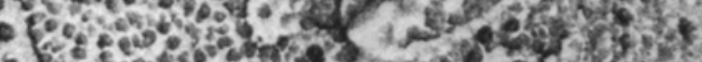
-

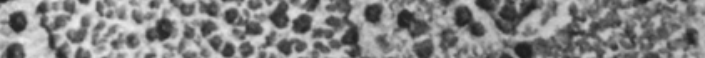

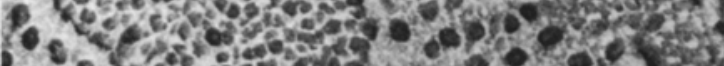

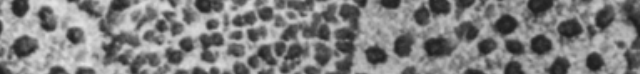

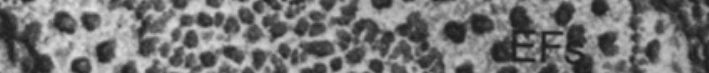

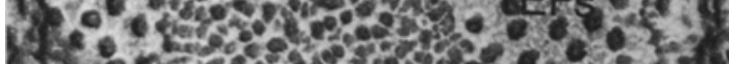

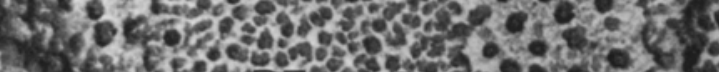

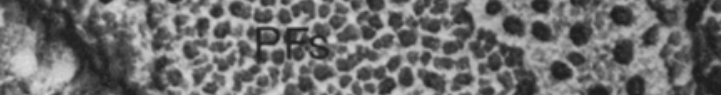

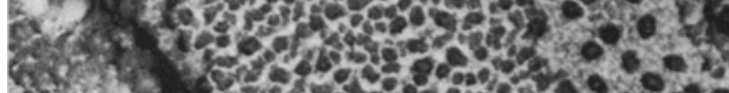

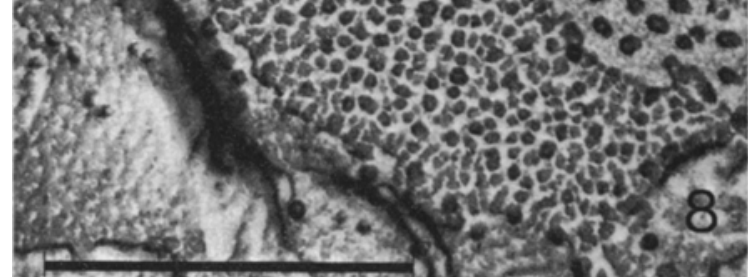

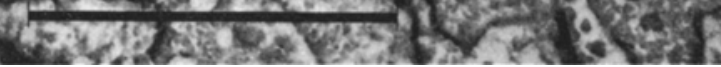

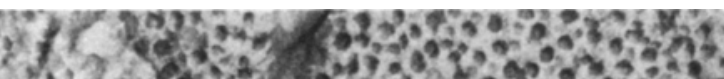

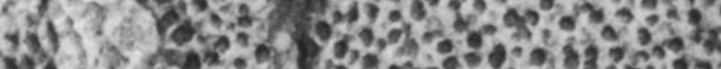

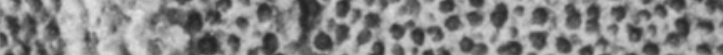

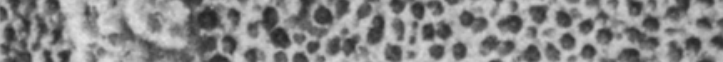

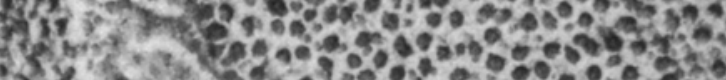
Sit $x$. 3.

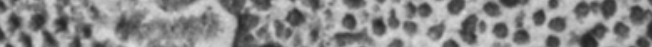

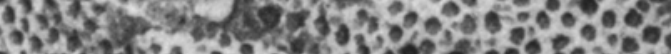

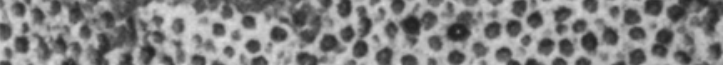

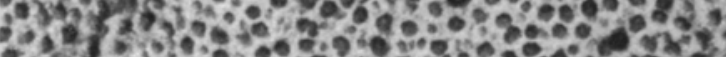

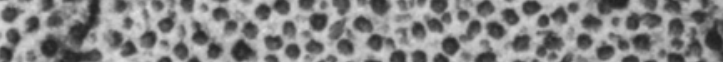

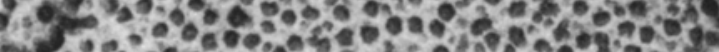

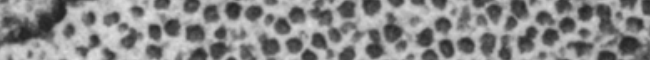

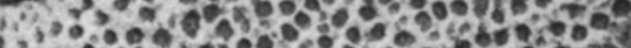

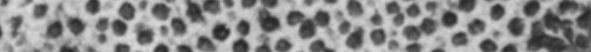
The

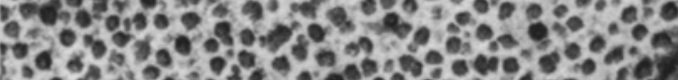
3oto

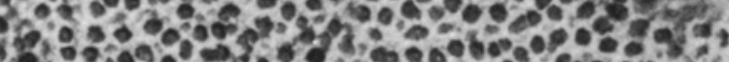

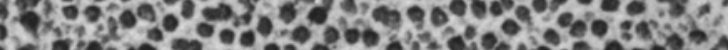

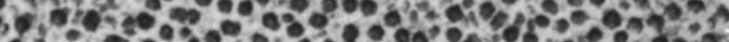

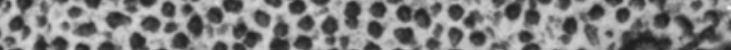
a Fis. Fo

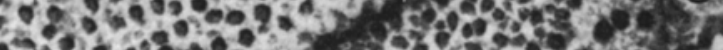


Figure 7. Enlargement of the EFs and EFu faces of wild-type barley thylakoids.

Note that particles of the EFs face vary in both size and shape and are more densely packed than those on the EFu face, with which it is continuous. The background of the EFu face is pitted with very small electrontransparent holes, in contrast to the smooth EFs face. $\times 150,000(\mathrm{Bar}=0.25 \mu \mathrm{m})$.

Figure 8. Micrograph showing the appearance of the PFs face of wild-type thylakoids and its relationship to the EFs face.

The particles on the PFs face are very densely packed. $\times 150,000($ Bar $=0.25 \mu \mathrm{m})$.

Figure 9. The PFu face of wild-type thylakoids is covered with particles that are slightly larger and less densely packed than those on the PFs face. $\times 150,000($ Bar $=0.25 \mu \mathrm{m})$.

\section{Table II}

Freeze-fracture particle densities of rotary shadowed wild-type and chlorina-f2 chloroplast thylakoids. S.E. $=$ standard error.

\begin{tabular}{|c|c|c|c|}
\hline Material & Face & No. $/ \mu \mathrm{m}^{2} \pm$ S.E. & Area measured $\left(\mu \mathrm{m}^{2}\right)$ \\
\hline \multirow[t]{4}{*}{ Wild-type } & $\mathrm{EFu}$ & $347 \pm 6$ & 6.69 \\
\hline & $\mathrm{PFu}$ & $4553 \pm 25$ & 1.28 \\
\hline & EFs & $1624 \pm 11$ & 1.83 \\
\hline & PFs & $6257 \pm 33$ & 1.02 \\
\hline \multirow[t]{4}{*}{ Chlorina- $f 2$} & EFu & $491 \pm 7$ & 7.34 \\
\hline & $\mathrm{PFu}$ & $4092 \pm 25$ & 1.28 \\
\hline & EFs & $2004 \pm 11$ & 5.59 \\
\hline & PFs & $1124 \pm 35$ & 0.53 \\
\hline
\end{tabular}

Table III

Freeze-fracture particles densities of uni-directionally shadowed wild-type and chlorina- $f 2$ chloroplast thylakoids. S.E. = standard error.

\begin{tabular}{llrc}
\hline Material & Face & No. $/ \mu \mathrm{m}^{2} \pm$ S.E. & Area measured $\left(\mu \mathrm{m}^{2}\right)$ \\
\hline Wild-type & $\mathrm{EFu}$ & $428 \pm 6$ & 8.61 \\
& $\mathrm{PFu}^{*}$ & $4378 \pm 36$ & 1.61 \\
& $\mathrm{EFs}^{*}$ & $1670 \pm 26$ & 1.66 \\
& $\mathrm{PFs}^{*}$ & $4662 \pm 49$ & 0.62 \\
Chlorina-f2 & & $558 \pm 8$ & 5.74 \\
& $\mathrm{EFu}$ & $3902 \pm 22$ & 1.54 \\
& $\mathrm{PFu}$ & $2103 \pm 12$ & 3.23 \\
& $\mathrm{EFs}$ & $1337 \pm 28$ & 0.77 \\
\hline
\end{tabular}

* Values from Simpson (35).

The EFu face, which is continuous with the EFs, was characterised by a low density of particles that were smaller and rounder than those on the EFs (Figure 7). In contrast to the smooth background of the EFs, the EFu face was pitted with numerous small holes which were seen as electron transparent spots. The PFu face (Figure 9) was covered with a large number of particles which were generally circular in outline and less densely packed than the particles on the PFs face.

The characteristics of rotary shadowed thyla- 
koids were quantified by measuring particle size and numbers of particles per square micron for each of the four fracture faces (Tables II and IV). Comparable data for uni-directionally shadowed material are given in Tables III and V and in (35). The EFu and EFs particle densities as measured from rotary shadowed specimens were respectively $19 \%$ and $3 \%$ lower than the values from uni-directionally shadowed replicas. The density of particles on the PFu face was $4 \%$ higher for rotary shadowed material and $34 \%$ higher for the PFs face when compared with the corresponding uni-directionally shadowed face (Tables II and III). The particle density on the unidirectionally shadowed EFu face was much lower than previously reported (35). The higher value found in earlier work (736 versus 347 particles $/ \mu \mathrm{m}^{-2}$ ) was probably due to movement of particles out of the EFs regions due to partial destacking under the lower ionic strength used ( $3 \mathrm{~mm}-\mathrm{Ca}\left(\mathrm{NO}_{3}\right)_{2}$ compared with $5 \mathrm{mM}-\mathrm{MgCl}_{2}$ in the present study).

The appearance of rotary shadowed freezefractured thylakoids from the mutant chlorina $-{ }^{2} 2$ can be seen at low magnification in Figure 10, which shows the relationship between three of the four fracture faces. The ultrastructure of the EFs and PFs faces shadowed uni-directionally is shown in Figure 11. The EFs face was covered with relatively large particles packed close together, lying on a smooth background. The PFs face had a small number of particles on a rough background containing pits and other irregularities (Figure 11). The EFs face, when examined by rotary shadowing (Figure 12), contained numerous particles which varied in both size and shape, ranging from elliptical to almost circular. The PFs face was characterised by a low density of approximately circular particles lying on an irregular background (Figure 12). It thus differed considerably from the equivalent face seen in wild-type thylakoids (Figure 8).

The EFu face of chlorina $f 2$ was identical to the corresponding face of wild-type thylakoids both for uni-directional (Figure 13) and rotary (Figure 14) shadowing. The same was true for the PFu face (Figures 15 and 16). This information is given in quantitative terms in Tables III and V and in Figure 17, where the particle densities, particle size and size distribu-
Table IV

Freeze-fracture particle sizes of rotary shadowed wild-type and chlorina- $f 2$ chloroplast thylakoids. S.D. = standard deviation.

\begin{tabular}{|c|c|c|c|}
\hline Face & Axis & $\begin{array}{l}\text { Average size } \\
\text { wild-type }\end{array}$ & $\begin{array}{l} \pm \text { S.D. }(\AA) \\
\text { chlorina- } f 2\end{array}$ \\
\hline $\mathrm{EFu}$ & $\begin{array}{l}\text { small } \\
\text { large }\end{array}$ & $\begin{array}{l}105 \pm 15 \\
126 \pm 18\end{array}$ & $\begin{array}{l}104 \pm 14 \\
121 \pm 18\end{array}$ \\
\hline $\mathrm{PFu}$ & $\begin{array}{l}\text { small } \\
\text { large }\end{array}$ & $\begin{array}{r}93 \pm 15 \\
112 \pm 18\end{array}$ & $\begin{array}{r}91 \pm 13 \\
109 \pm 16\end{array}$ \\
\hline EFs & $\begin{array}{l}\text { small } \\
\text { large }\end{array}$ & $\begin{array}{l}117 \pm 13 \\
155 \pm 20\end{array}$ & $\begin{array}{l}104 \pm 13 \\
138 \pm 21\end{array}$ \\
\hline PFs & $\begin{array}{l}\text { small } \\
\text { large }\end{array}$ & $\begin{array}{l}74 \pm 12 \\
93 \pm 14\end{array}$ & $\begin{array}{r}90 \pm 15 \\
103 \pm 17\end{array}$ \\
\hline
\end{tabular}

tion of uni-directionally shadowed chlorina-f2 thylakoids can be compared with data from wildtype thylakoids. The data for rotary shadowed chlorina- $f 2$ thylakoids is presented in Tables II and IV. The main difference between wild-type and mutant thylakoids was found on the PFs and EFs faces. The mutant PFs face had a greatly reduced number of particles $\left(1124 / \mu^{2}\right.$ versus $6257 / \mu^{2}$ for wild-type), and these particles were slightly larger than those on the wild-type PFs face (Tables IV and V). The EFs face of chlorina- $f 2$ thylakoids contained a higher density of particles than the wild-type $\left(2004 / \mu \mathrm{m}^{2}\right.$ versus $1624 / \mathrm{mm}^{2}$ ) and these particles were on average $12 \%$ smaller than the wild-type EFs face particles (Table IV). There were some small differences in the particle densities and sizes on

\section{Table V}

Freeze-fracture particle sizes of uni-directionally shadowed wild-type and chlorina- $f 2$ chloroplast thylakoids. S.D. = standard deviation.

\begin{tabular}{lrr}
\hline Face & \multicolumn{2}{c}{$\begin{array}{c}\text { Average size } \pm \text { S.D. }(\AA) \\
\text { wild-type }\end{array}$} \\
\hline $\mathrm{EFu}$ & $97 \pm 17$ & $102 \pm 19$ \\
$\mathrm{PFu}$ & $101 \pm 27^{*}$ & $94 \pm 16$ \\
$\mathrm{EFs}$ & $144 \pm 29^{*}$ & $126 \pm 26$ \\
$\mathrm{PFs}$ & $81 \pm 13^{*}$ & $89 \pm 18$ \\
\hline
\end{tabular}

* From Simpson (35). 


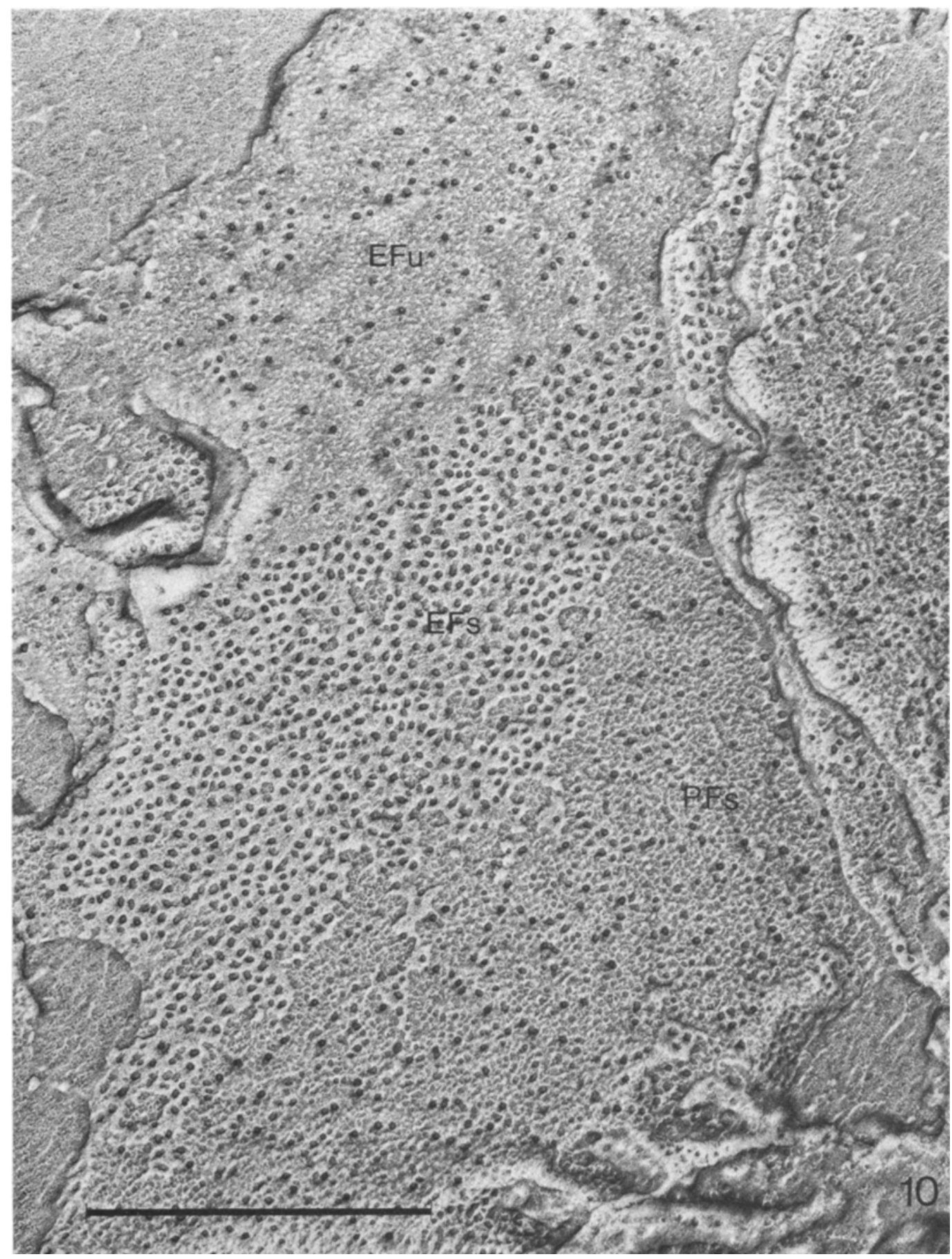

Figure 10. Rotary shadowed freeze-fractured thylakoids of chlorina- 2 chloroplasts showing the relationship between the PFs. EFs and EFu faces.

Note the large areas of fractured membranes from grana (EFs and PFs) in comparison with wild-type. $\times 100,000(\mathrm{Bar}=0.5 \mu \mathrm{m})$. 


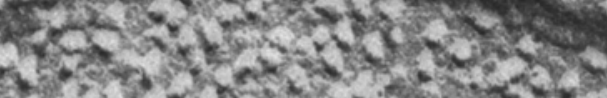

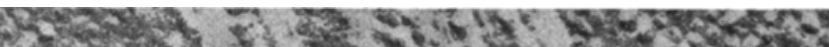

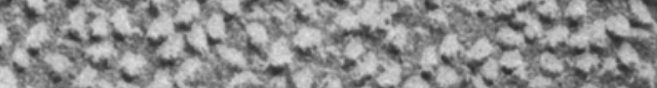

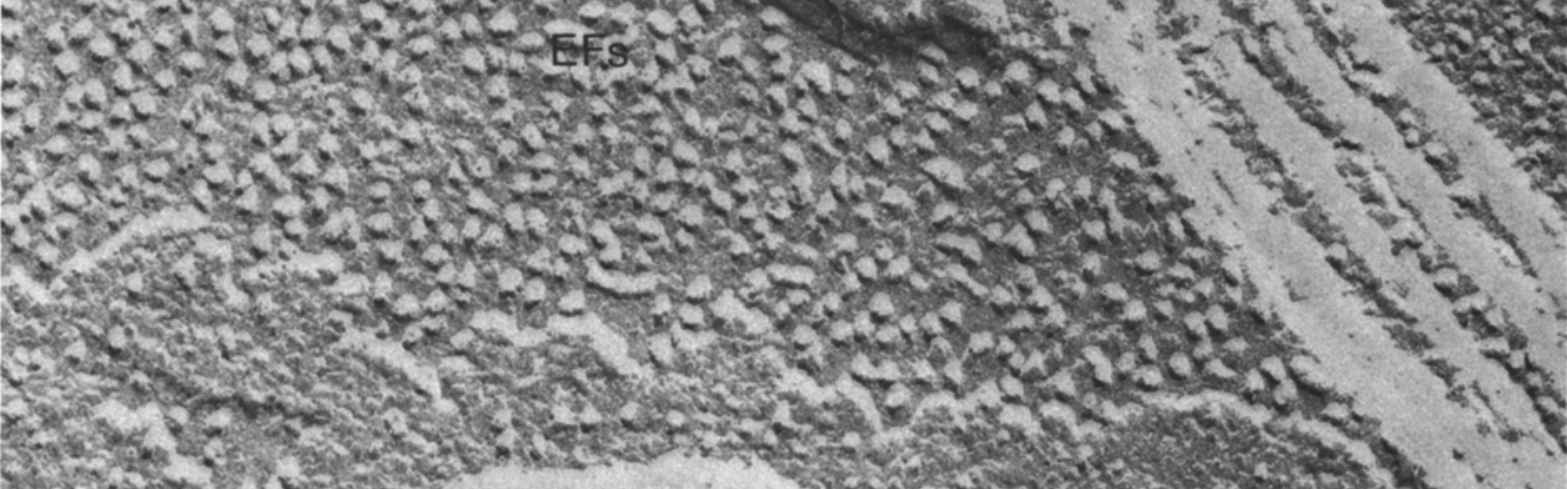
W.

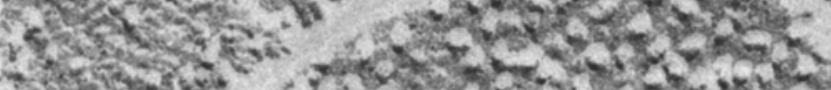

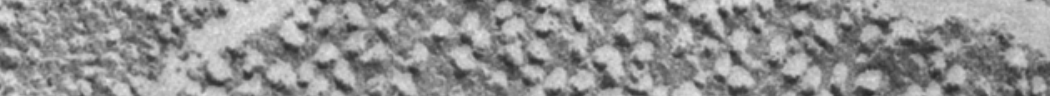

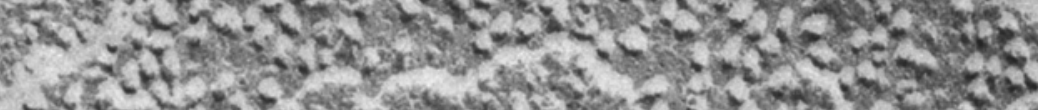

15.6.

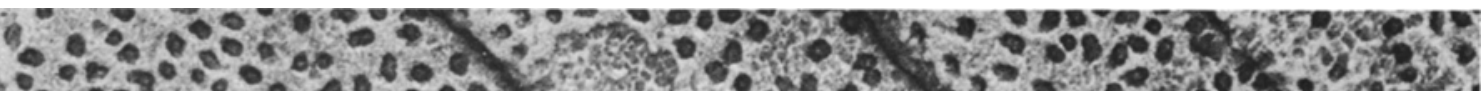

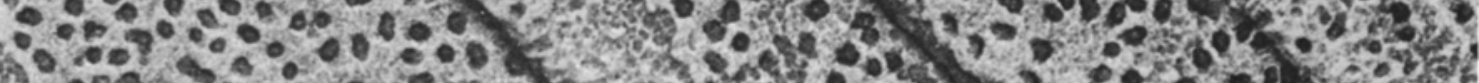

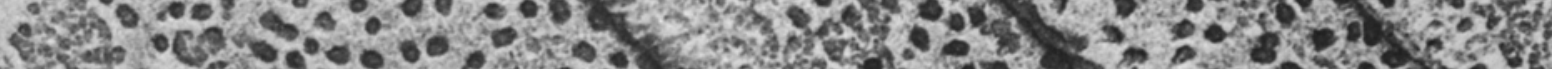

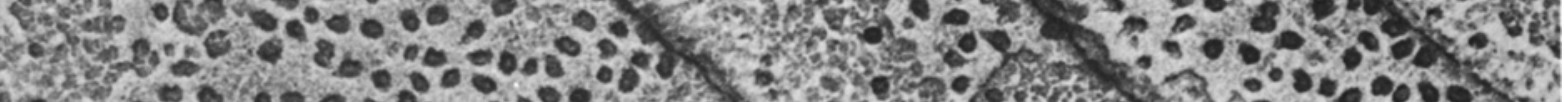

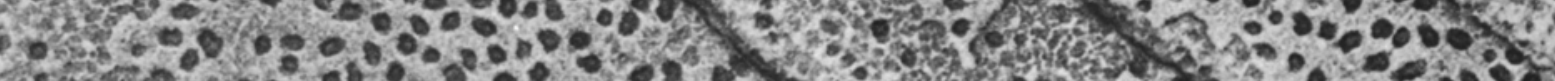

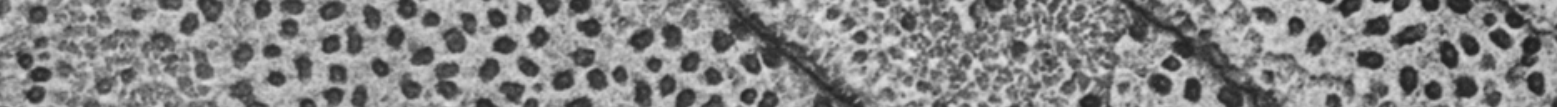

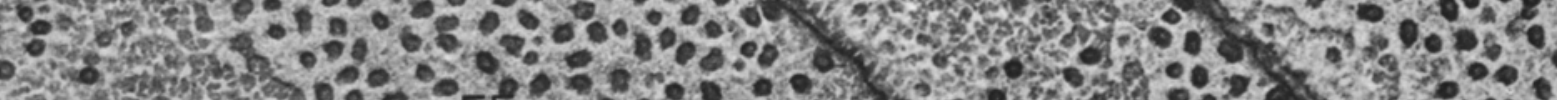

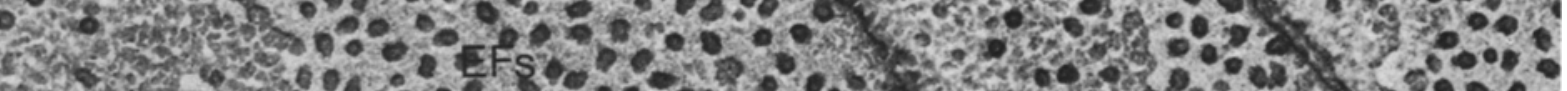

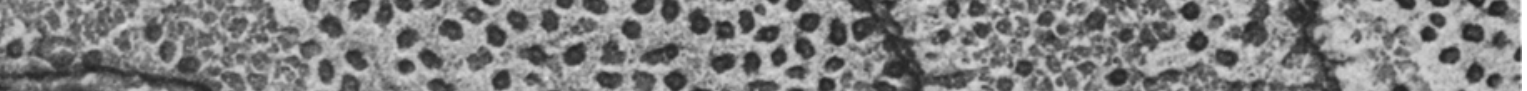

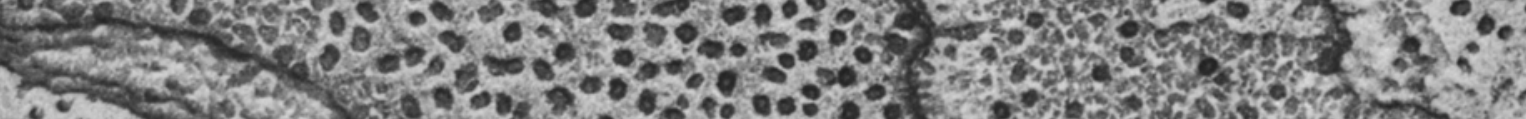

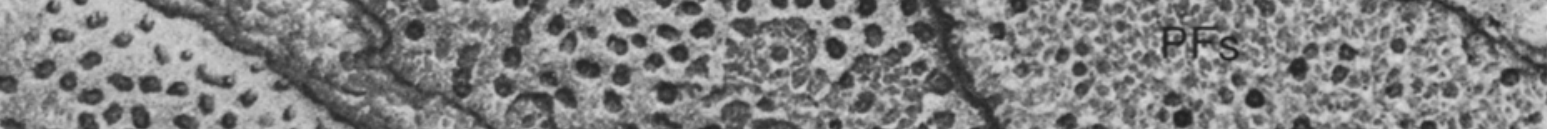

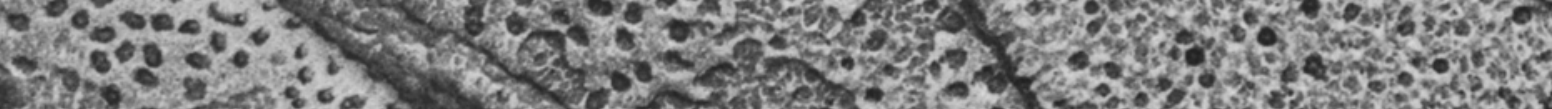

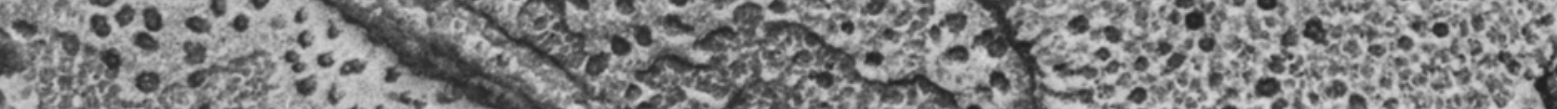

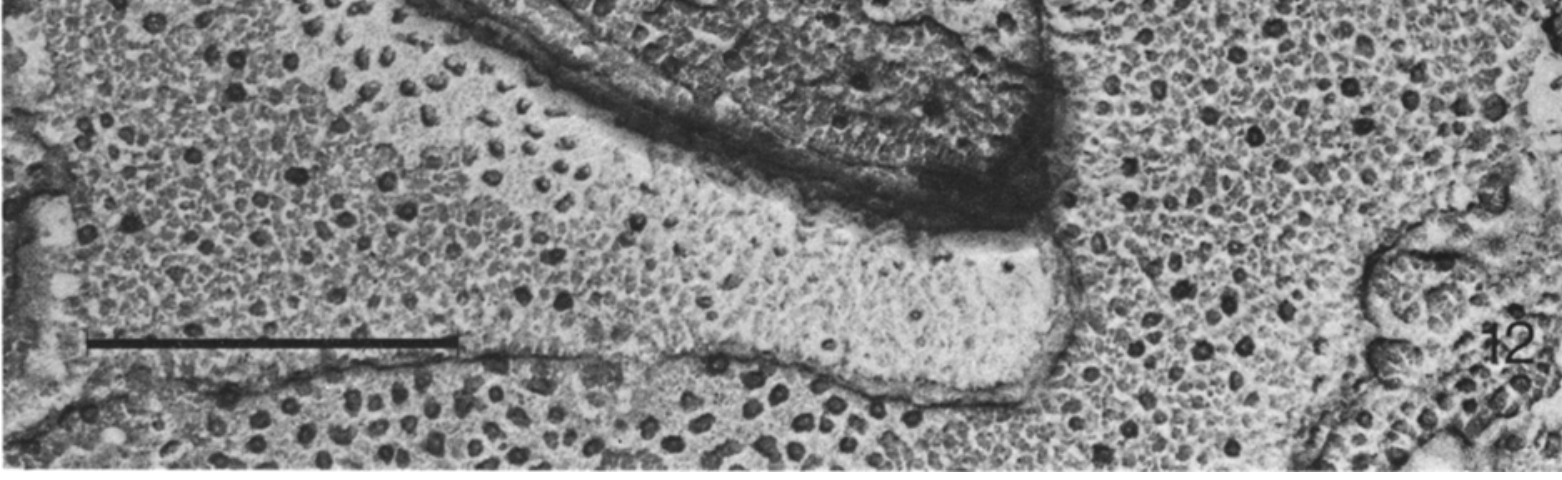




\section{$\mathrm{EFu}$}

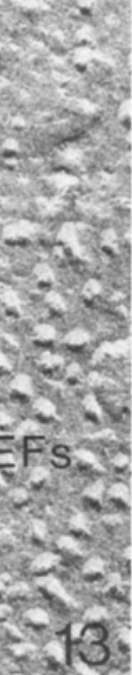

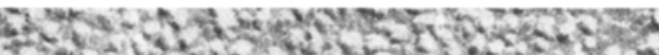

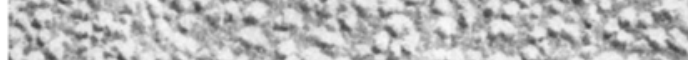

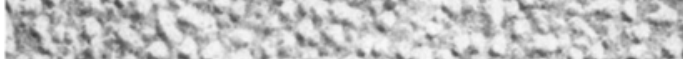

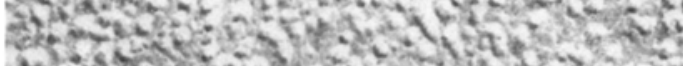

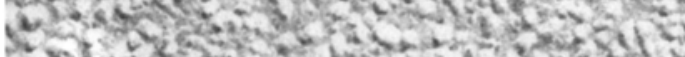

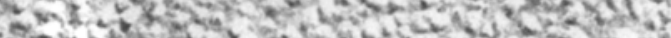

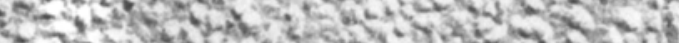

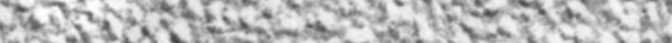

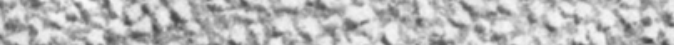

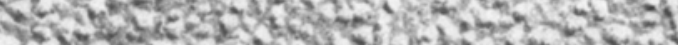

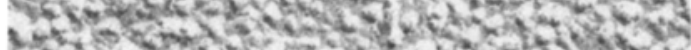

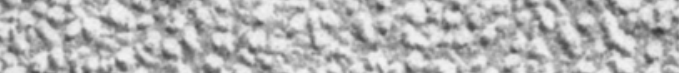

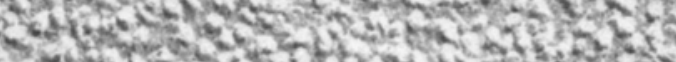

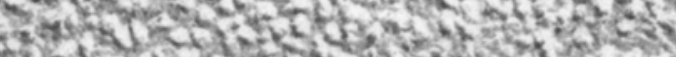

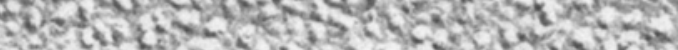

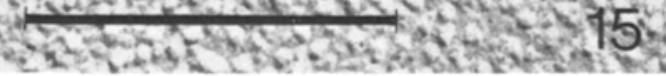

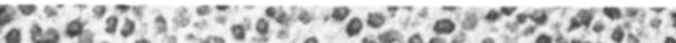

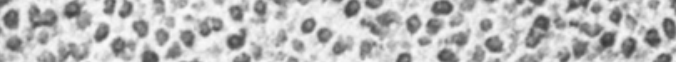

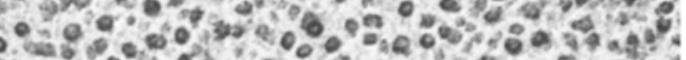

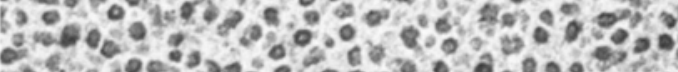

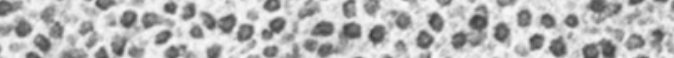

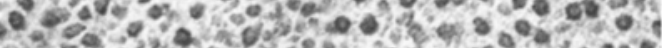

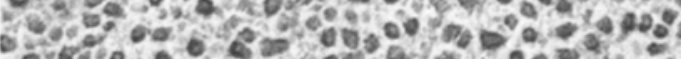

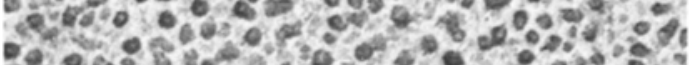

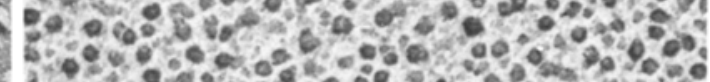

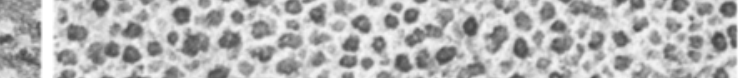

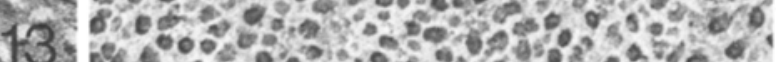

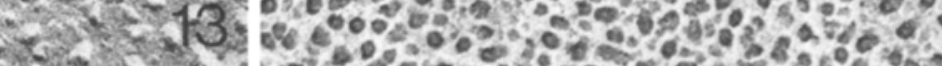

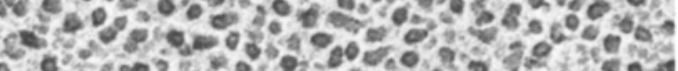

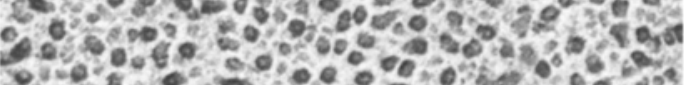

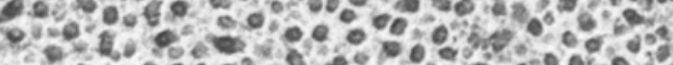

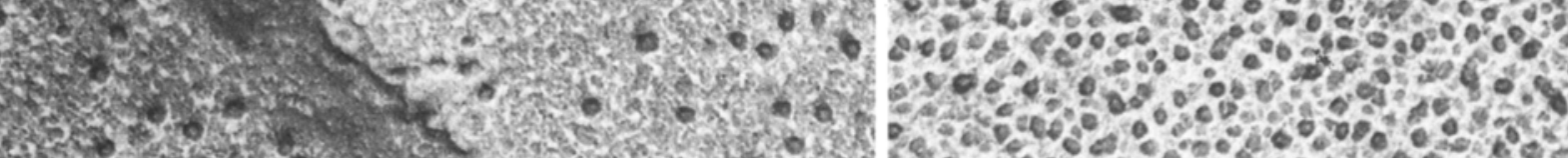

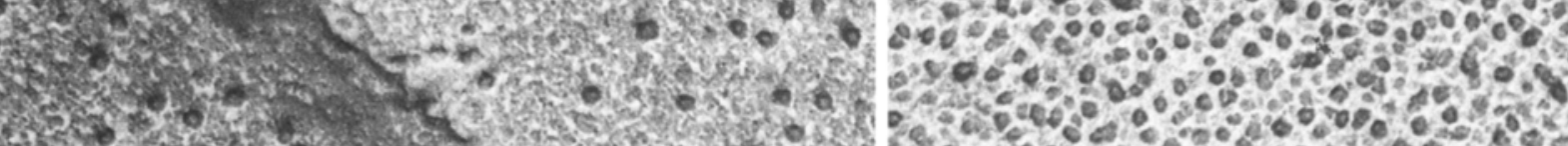

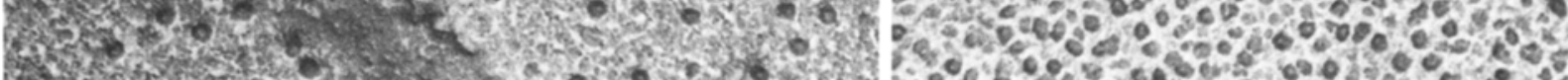

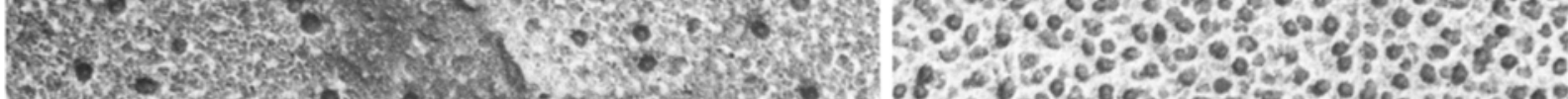

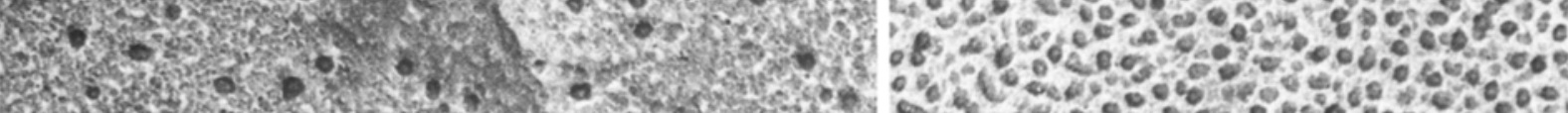

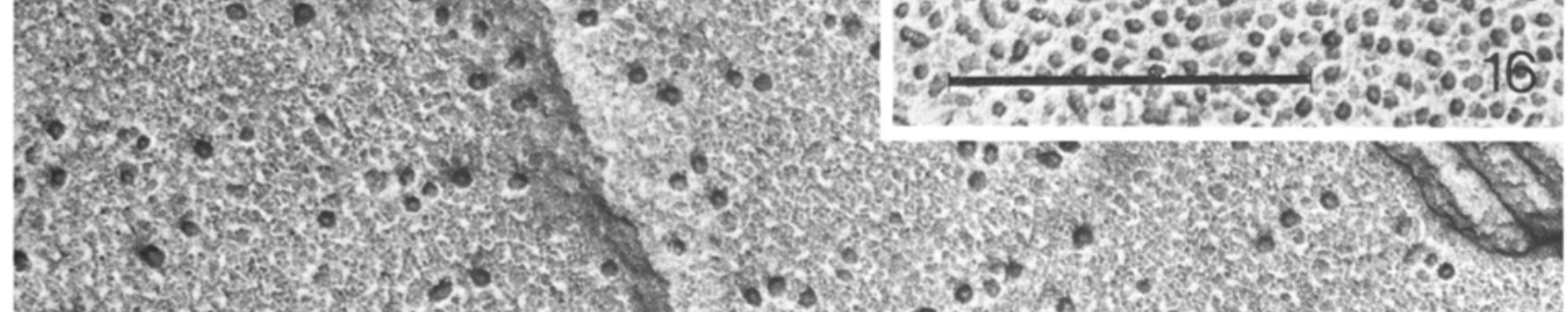

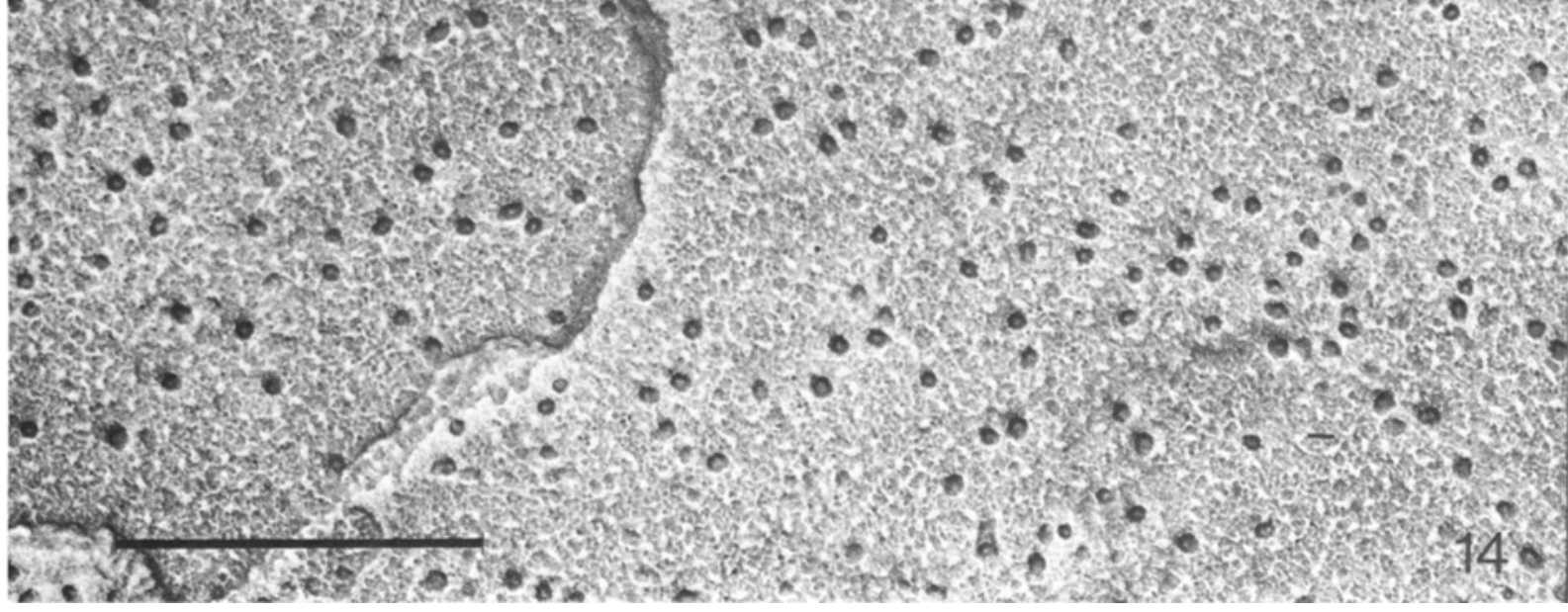


D. J. Simpson: Freeze-fractured barley thylakoids

Figure 11. Uni-directionally shadowed freeze-fractured chlorina- $f$ thylakoids showing the appearance of the EFs and PFs faces.

The PFs face contains a low density of particles on a rough background. $\times 150,000($ Bar $=0.25 \mu \mathrm{m})$.

Figure 12. Rotary shadowed freeze-fractured chlorina- 2 thylakoids from the preparation used in Figure 11 , showing the EFs and PFs faces.

These may be compared with the corresponding wild-type faces in Figures 7 and $8 . \times 150,000(B a r=$ $0.25 \mu \mathrm{m})$.

Figure 13. Uni-directionally shadowed freeze-fractured chlorina- $f 2$ thylakoids showing the appearance of the EFu face. $\times 150,000($ Bar $=0.25 \mu \mathrm{m})$.

Figure 14. As in Figure 13, showing the EFu face after freeze-fracturing followed by rotary shadowing. $\times 150,000($ Bar $=0.25 \mu \mathrm{m})$.

Figure 15. The PFu face from uni-directionally shadowed freeze-fractured chlorina- $f 2$ thylakoids. $\times 150,000$ $($ Bar $=0.25 \mu \mathrm{m})$.

Figure 16. The PFu face from the same material as in Figure 15 after rotary shadowing.

There is no significant difference between the EFu and PFu faces of wild-type and chlorina- $f 2$ thylakoids. $\times 150,000($ Bar $=0.25 \mu \mathrm{m})$.

\section{Table VI}

Characteristics of rotary shadowed thylakoid surfaces of wild-type and chlorina-f2. S.D. = standard deviation. S.E. = standard error.

\begin{tabular}{|c|c|c|c|}
\hline \multirow[t]{2}{*}{ Surface } & \multirow[t]{2}{*}{ Axis } & \multicolumn{2}{|c|}{ Particle size \pm S.D. $(\AA)$} \\
\hline & & wild-type & chlorina-f2 \\
\hline $\mathrm{PSu}$ & $\begin{array}{l}\text { small } \\
\text { large }\end{array}$ & & $\begin{array}{r}99 \pm 13 \\
120 \pm 14\end{array}$ \\
\hline ESs & $\begin{array}{l}\text { small } \\
\text { large }\end{array}$ & $\begin{array}{l}142 \pm 11 \\
191 \pm 16\end{array}$ & $\begin{array}{l}122 \pm 11 \\
174 \pm 15\end{array}$ \\
\hline No. $/ \mu \mathrm{m}^{2}$ & S.E. & $1450 \pm 15$ & $2054 \pm 17$ \\
\hline ESs array & $\begin{array}{l}\text { small } \\
\text { large }\end{array}$ & $\begin{array}{l}130 \pm 10 \\
195 \pm 11\end{array}$ & $\begin{array}{l}115 \pm 10 \\
178 \pm 9\end{array}$ \\
\hline Centre-to-c & ntre spacings & $152 \times 237(\AA)$ & $145 \times 207(\AA)$ \\
\hline
\end{tabular}

Table VII

Characteristics of wild-type and chlorina $-f 2$ grana. Values are \pm standard deviation.

\begin{tabular}{lcr}
\hline Parameter & wild-type & chlorina- $f 2$ \\
\hline Diameter $(\mu \mathrm{m})$ & $0.42 \pm 0.05$ & $1.01 \pm 0.29$ \\
No. of discs & $10.4 \pm 4.8$ & $8.5 \pm 3.4$ \\
Centre-to-centre spacing & & \\
$\quad$ thin sections $(\AA)$ & $161.7 \pm 5.4$ & $196.9 \pm 5.9$ \\
$\quad$ freeze-fracture $(\AA)$ & $272.5 \pm 7.4$ & $267.7 \pm 13.8$ \\
Partition width $(\AA)$ & $193.8 \pm 19.2$ & $200.3 \pm 16.4$ \\
Intrathylakoid space width $(\AA)$ & $79.3 \pm 14.4$ & $64.2 \pm 12.1$ \\
\hline
\end{tabular}




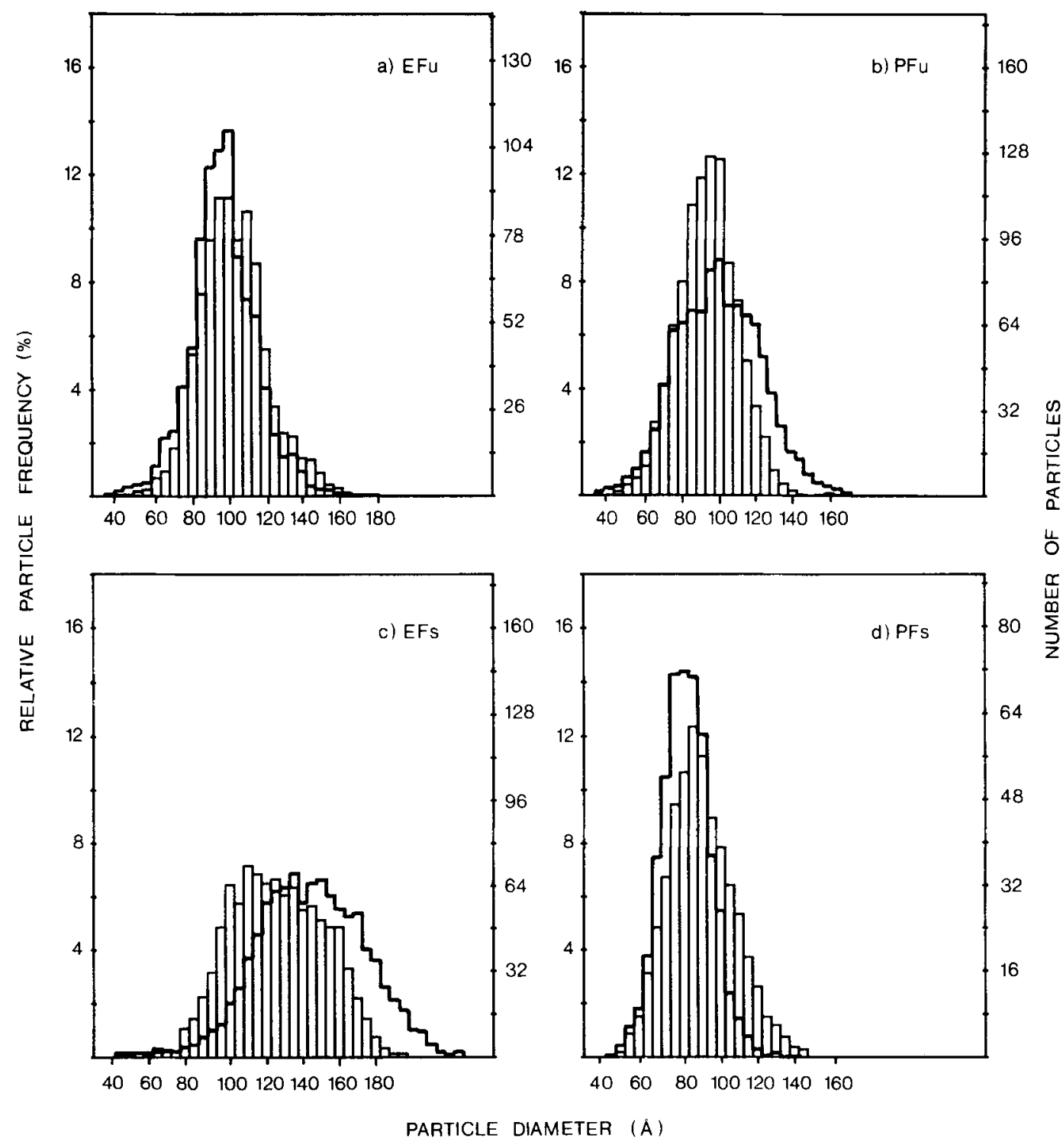

Figure 17. Computer-drawn histogram plots of the size distribution of the particles on the four different fracture faces of uni-directionally shadowed chlorina- $f 2$ thylakoids (light lines) compared with those of the corresponding faces of wild-type thylakoids (heavy lines).

Numerical values are summarised in Table $\mathrm{V}$.

Figure 18. The ESs of wild-type thylakoids after etching and rotary shadowing.

The tetrameric particles sometimes form arrays in stacked thylakoids. The average size of such particles is $130 \times 195 \AA$ with centre-to-centre spacings of $152 \times 237 \AA . \times 300,000(\mathrm{Bar}=0.1 \mu \mathrm{m})$.

Figure 19. Rotary shadowed ESs array of chlorina-f2 thylakoids.

Particles appear very similar to wild-type, having a tetrameric substructure and similar surface relief, with an average size of $115 \times 172 \AA$ and centre-to-centre spacings of $145 \times 207 \AA . \times 300.000(\mathrm{Bar}=0.1 \mu \mathrm{m})$. 


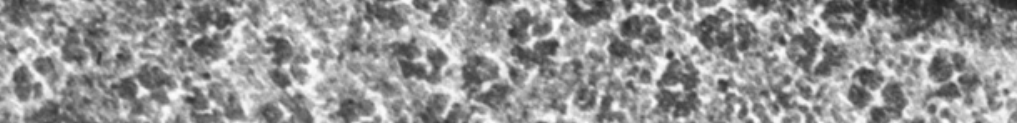
F.

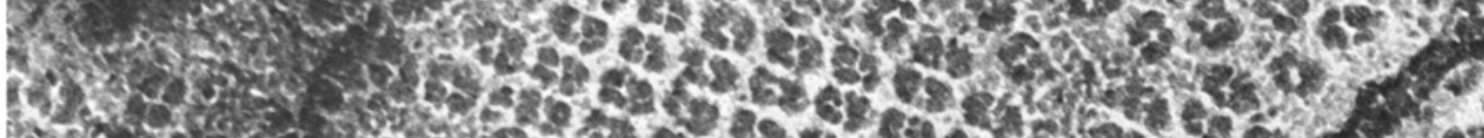

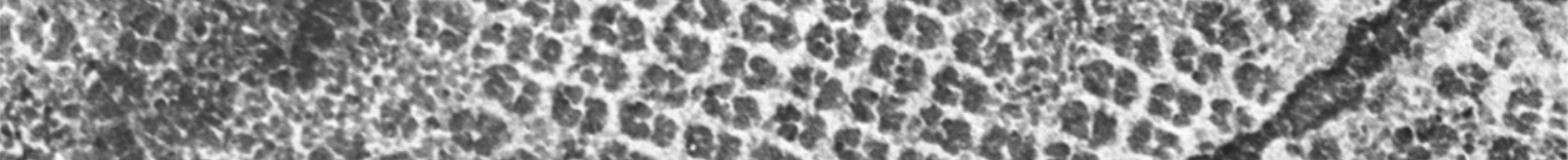

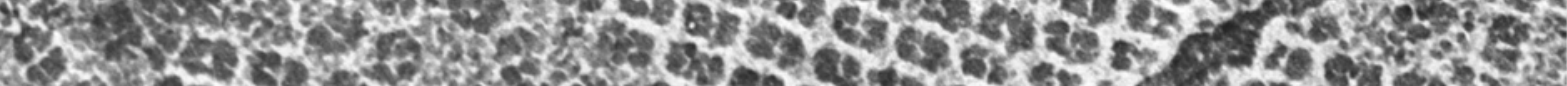

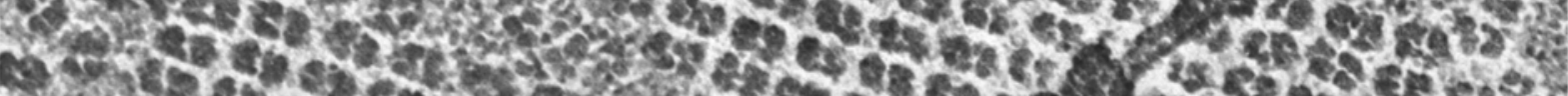

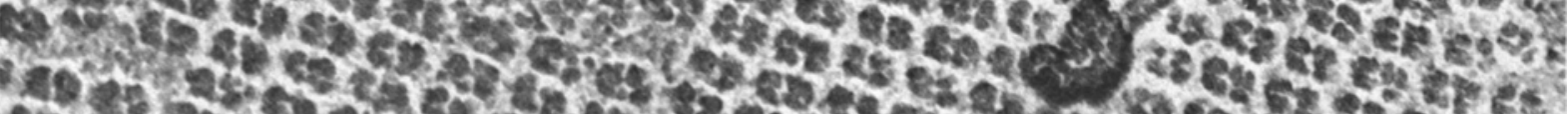

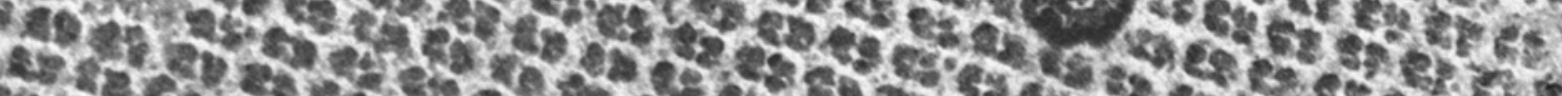

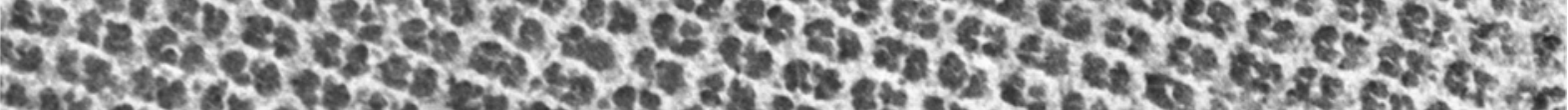

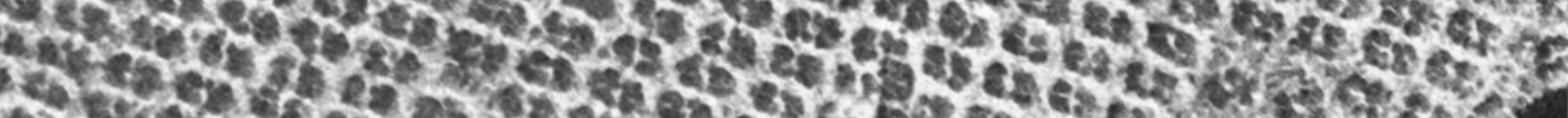
6.

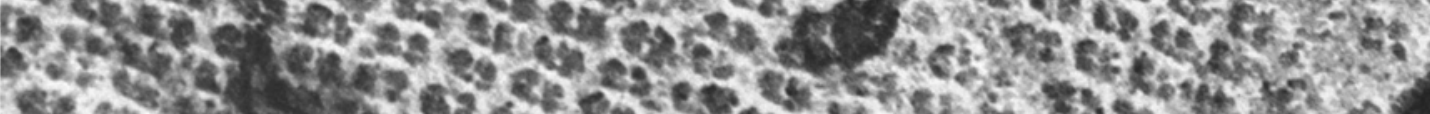

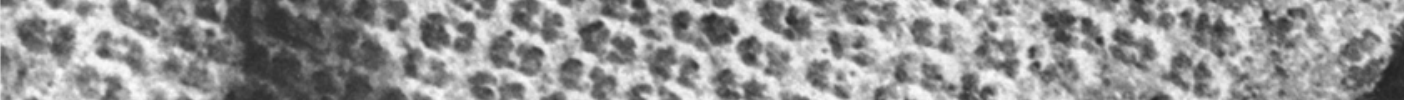

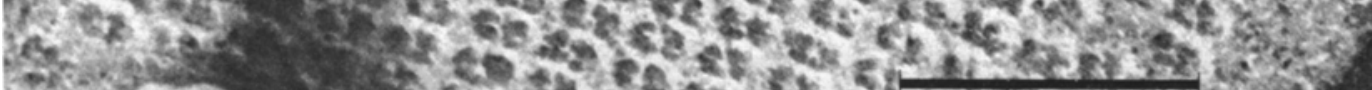

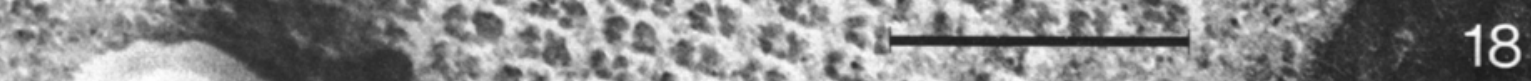

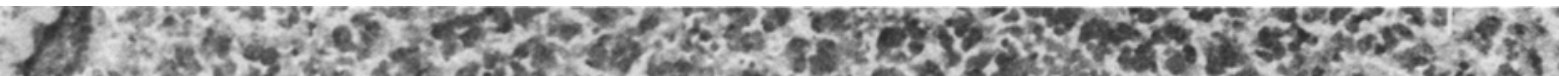

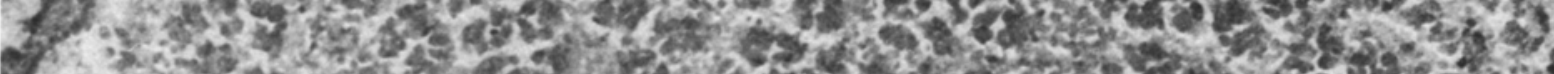

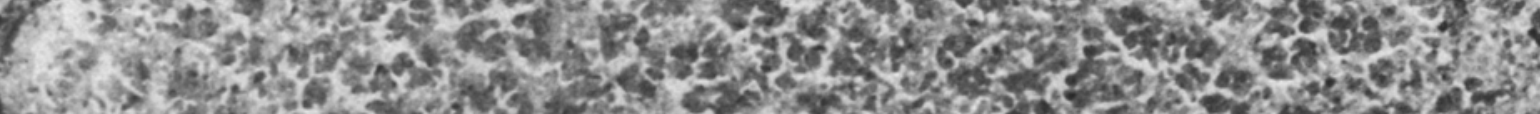
4 -

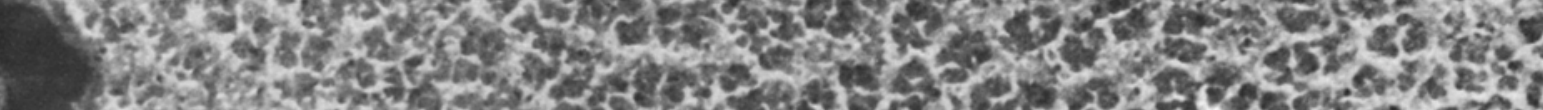

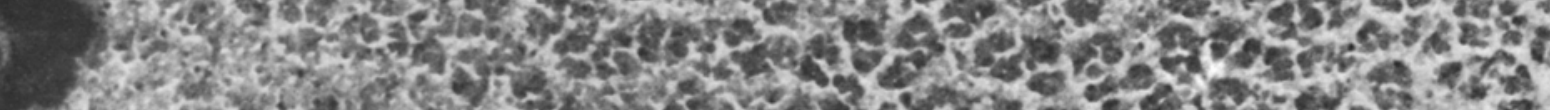

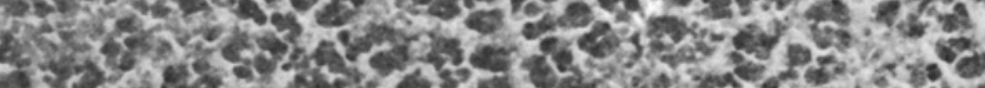
25
2

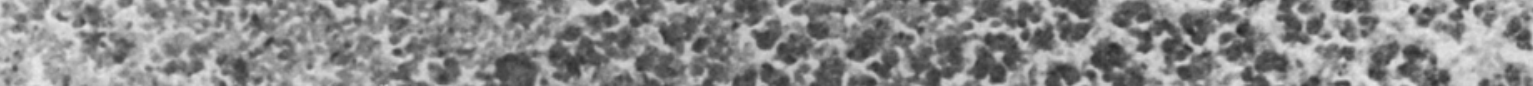

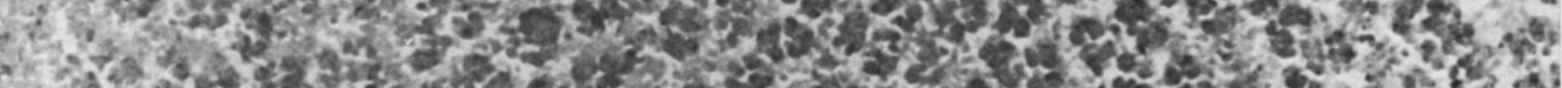

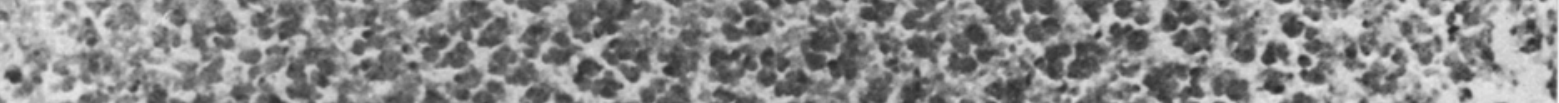

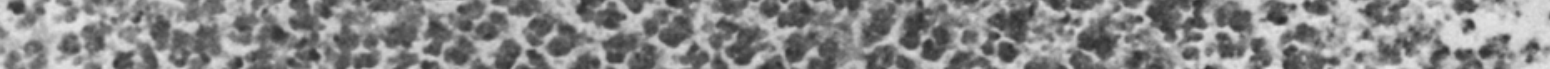

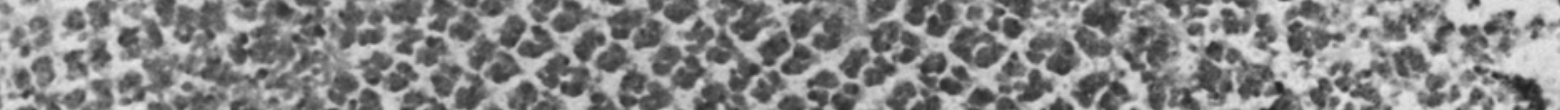

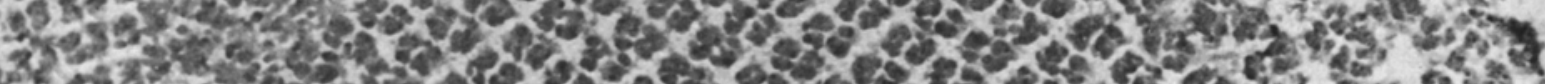

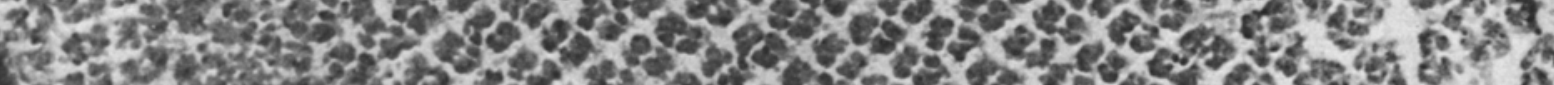

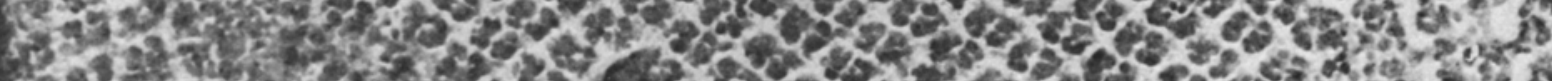

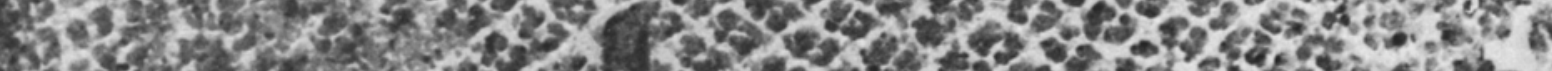

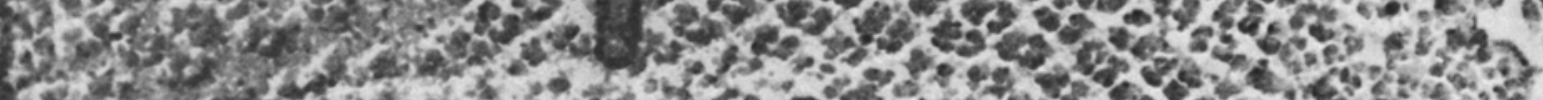

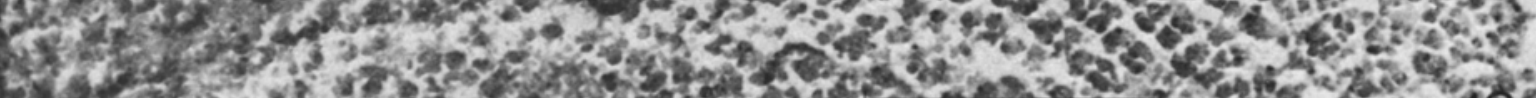

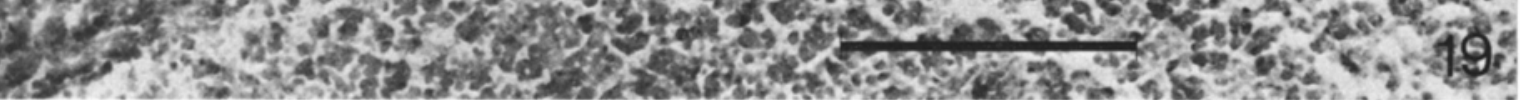




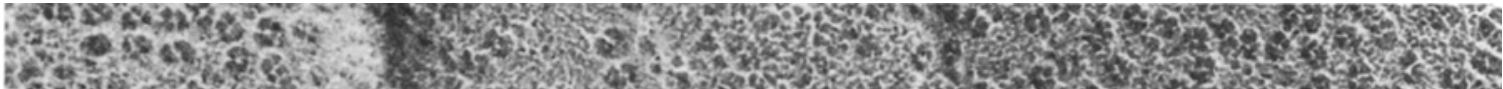

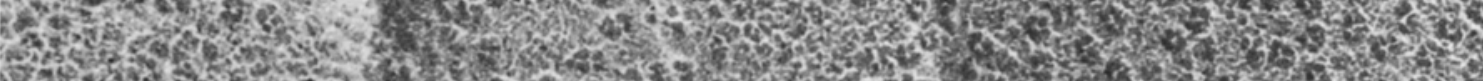

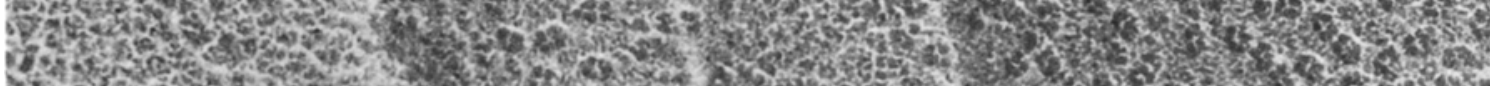

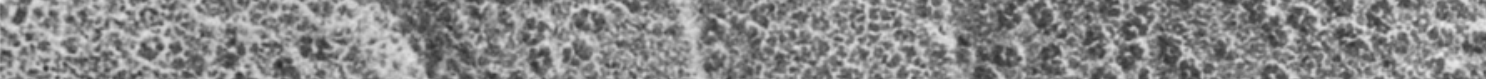

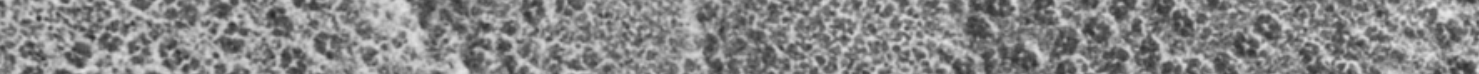

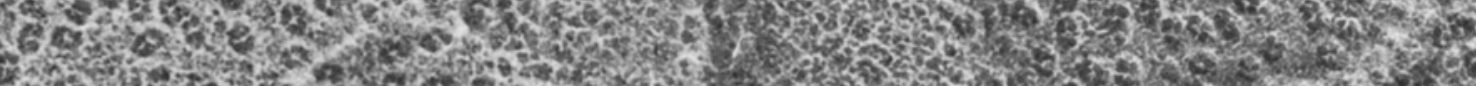

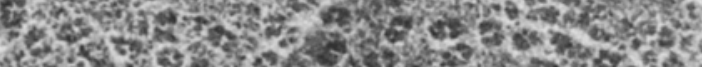

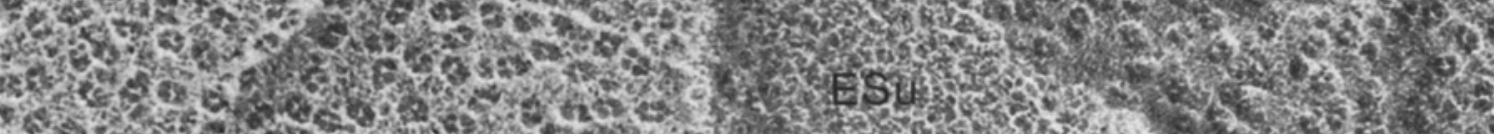

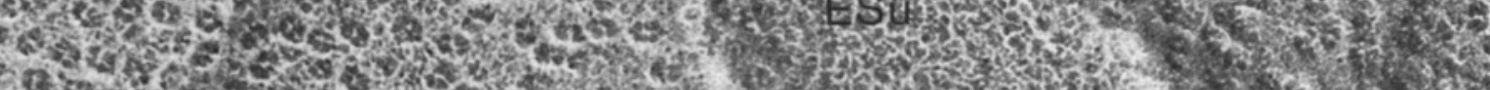
5.t.

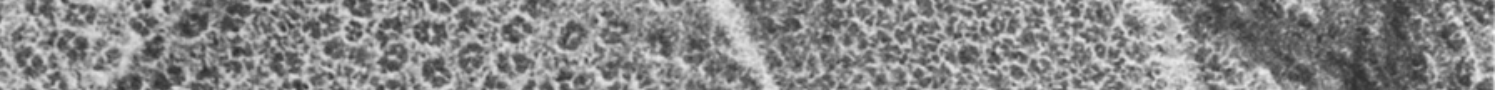

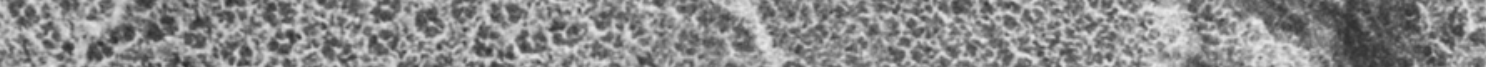

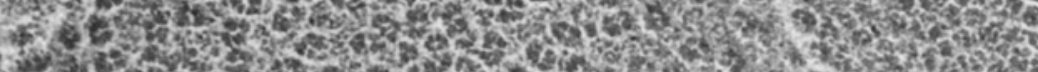

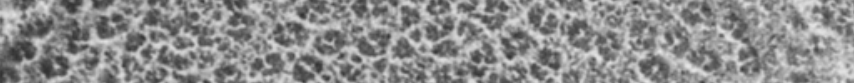

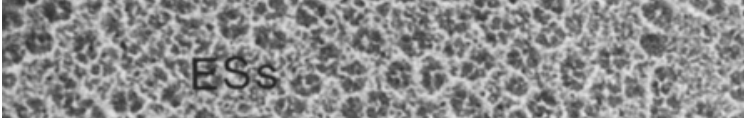

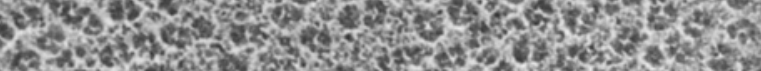

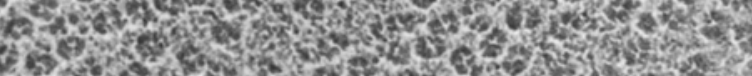

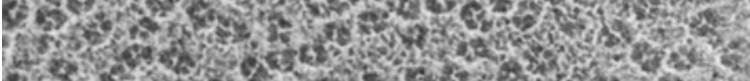

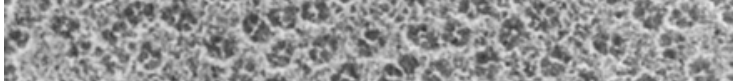

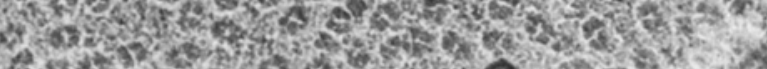

o.

sted

$6 \sqrt{2}=$

in:

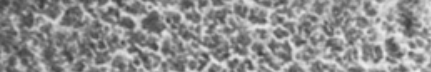

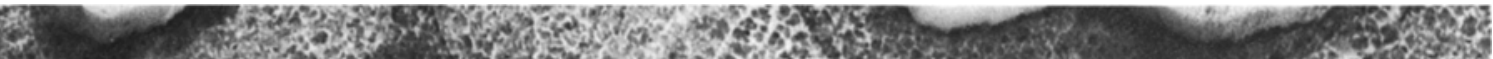

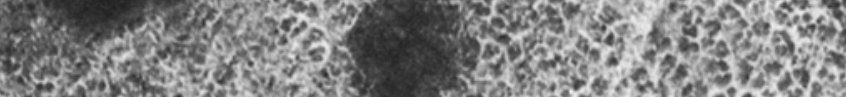

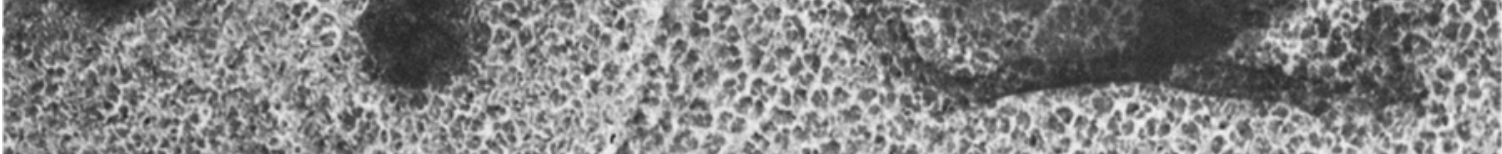

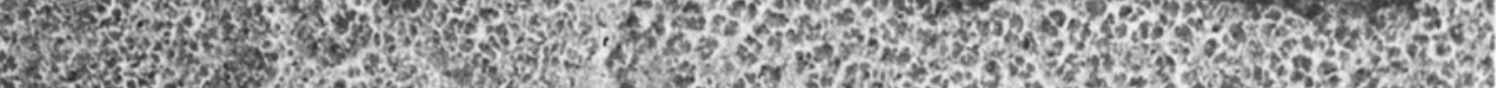

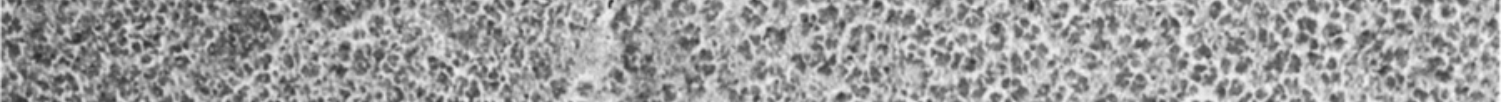

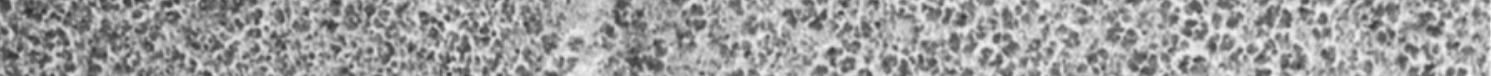

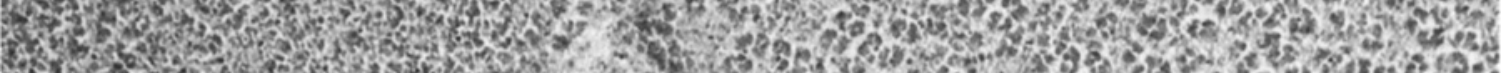

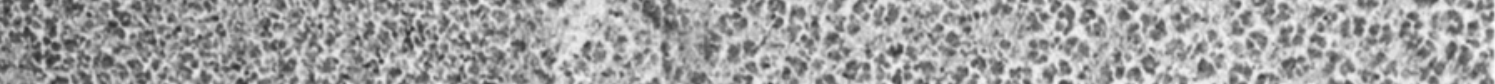

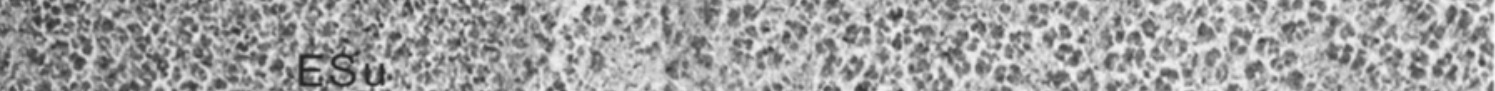

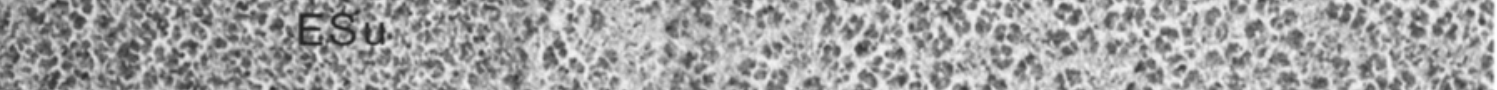

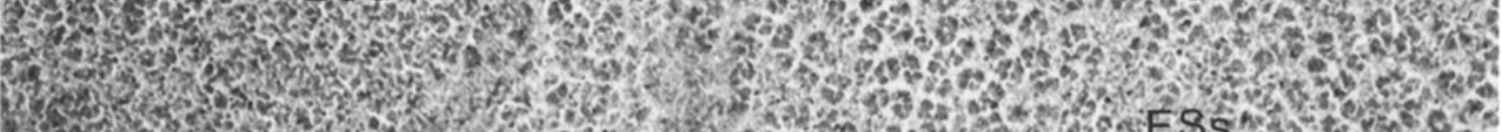

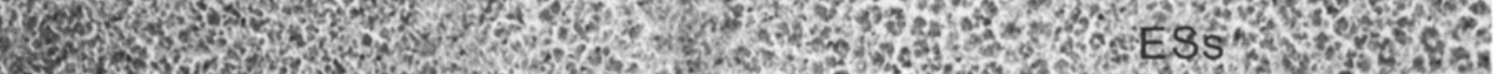

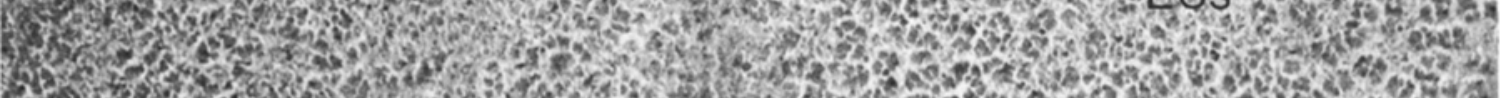

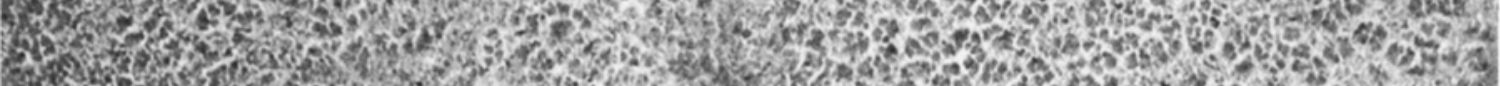

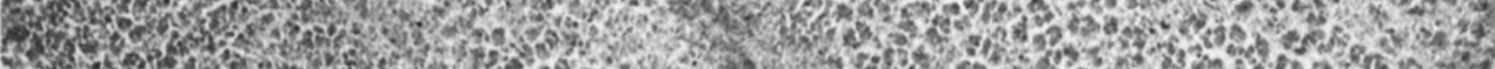

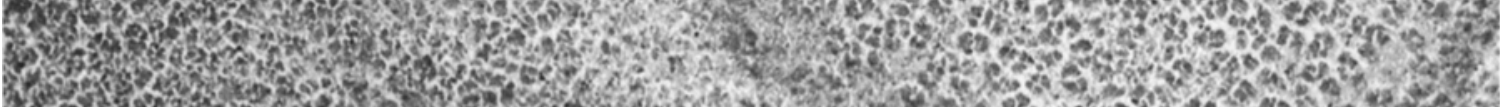

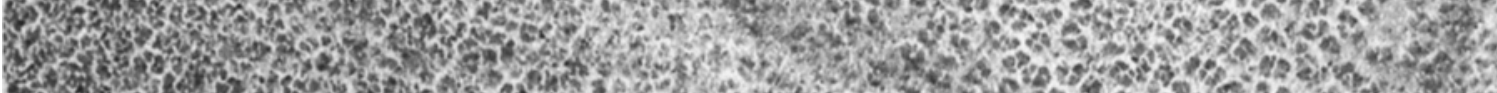

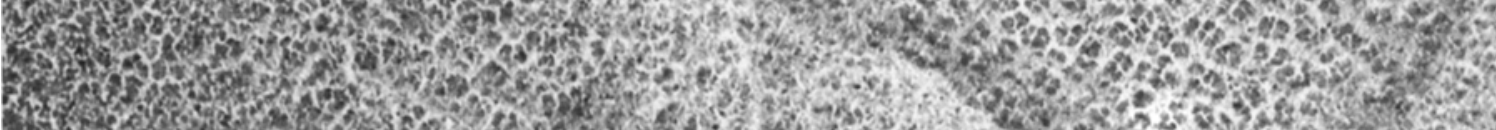

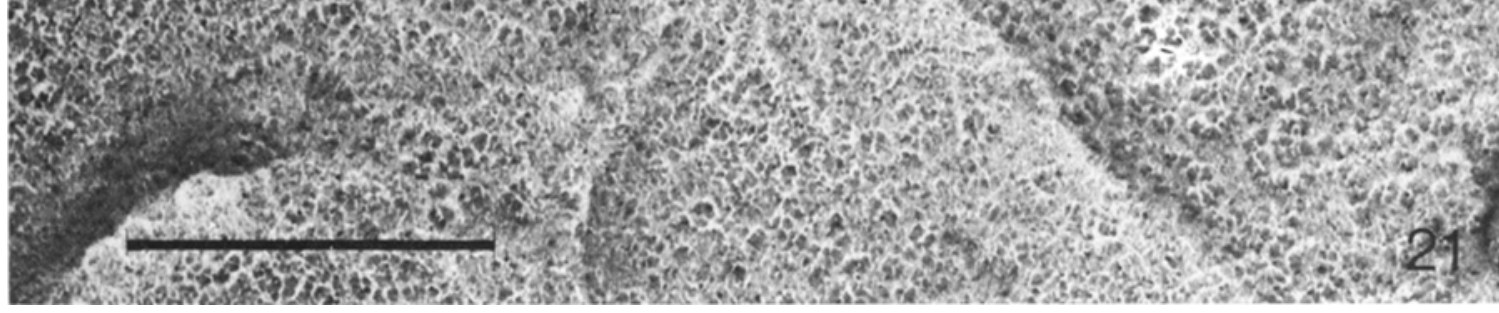


Figure 20. Rotary shadowed ESs and ESu of wild-type thylakoids.

The ESs is covered with tetrameric particles with an average size of $142 \times 191 \AA$. The ESu has a particulate appearance suggesting the presence of numerous particles, but these are difficult to resolve clearly from one another. $\times 150,000(\mathrm{Bar}=0.25 \mu \mathrm{m})$.

Figure 21 . The ESs and ESu of chlorina $-f 2$ thylakoids after etching and rotary shadowing.

As found for wild-type, the ESs is covered with tetrameric particles, with an average size of $122 \times 174 \AA$ and a density close to that of EFs particles. The appearance of the ESu indicates that it is covered with particles of a low surface relief. $\times 150,000(\mathrm{Bar}=0.25 \mu \mathrm{m})$.

the $\mathrm{EFu}$ and $\mathrm{PFu}$ faces of mutant thylakoids compared with wild-type (Tables II and IV), but these were small in comparison with those found for the PFs and EFs faces.

Since it is thought that ESs particles correspond to the intramembranous EFs particles (28, 30 ), the nature of these particles on mutant and wild-type thylakoids was also investigated by rotary shadowing. These particles were sometimes found in arrays in stacked thylakoids of wild-type (Figure 18) and chlorina-f2 (Figure 19). The centre-to-centre spacings of these arrays (Table VI) revealed that chlorina-f2 ESs particles were spaced slightly closer together than those of wild-type, mainly in the longer dimension. The size of particles in chlorina- $f 2$ ESs arrays (Table VI), were $13 \%$ smaller than the wild-type, which was the same as the difference in size of the EFs particles. A similar size difference was found for ESs particles not in arrays, which were slightly wider than the particles in arrays (Table VI). The size of the ESs particles was much more uniform than the EFs particles, although the number of particles per square micron was close to that found for the EFs face (Tables II, IV and VI).

The majority of ESs particles, both in arrays and not arrayed, in wild-type and chlorina-f2 thylakoids, had a tetrameric substructure (Figures 18-21). The relationship between these particles and PFs particles can be seen in Figure 22 in which the rows of ESs particles coincide with the rows of grooves between PFs particles. The ESs of chlorina- $f 2$ thylakoids occasionally contained particles in a square lattice (Figure 23) similar to those on the ESs of wild-type thylakoids (35). A close examination of the ESu of wild-type (Figure 20) and mutant (Figure 21) thylakoids revealed no tetrameric particles. Instead there were a large number of particles of low surface relief, which were much smaller than those seen on the ESs. These particles were not seen in specimens shadowed at $45^{\circ}$ from one direction $(35,39)$.

The PSu faces of wild-type and chlorina- $f 2$ thylakoids were essentially the same, being covered with many large and small particles (Figure 24). The large particles, thought to correspond to coupling factor 1 and ribulose-1,5bisphosphate carboxylase molecules (39) were often clustered into groups of 5 to 15 particles. The average size of the large particles was determined to be about $100 \times 120 \AA$ (Table VI).

When transversely fractured rotary shadowed thylakoids were examined, it was possible to distinguish the partition region from the intra-

Figure 22. Etched preparation of de-stacked thylakoids of wild-type barley demonstrating that the grooves between double rows of PF particles correspond with the rows of ES particles. $\times 300,000(\mathrm{Bar}=0.1 \mu \mathrm{m})$.

Figure 23. This type of lattice was occasionally seen on the ES of chlorina $-f 2$ thylakoids, and has previously been found on wild-type thylakoids $(35) \times 300,000(B a r=0.1 \mu \mathrm{m})$.

Figure 24. Rotary shadowed PSu of chlorina- $f 2$ thylakoids.

The large particles, thought to be the coupling factor 1 and ribulose-1,5-bisphosphate carboxylase, measure about $100 \times 120 \AA$ and often occur in small clusters. There are also many smaller particles present. $\times 300,000$ $(\operatorname{Bar}=0.1 \mu \mathrm{m})$. 


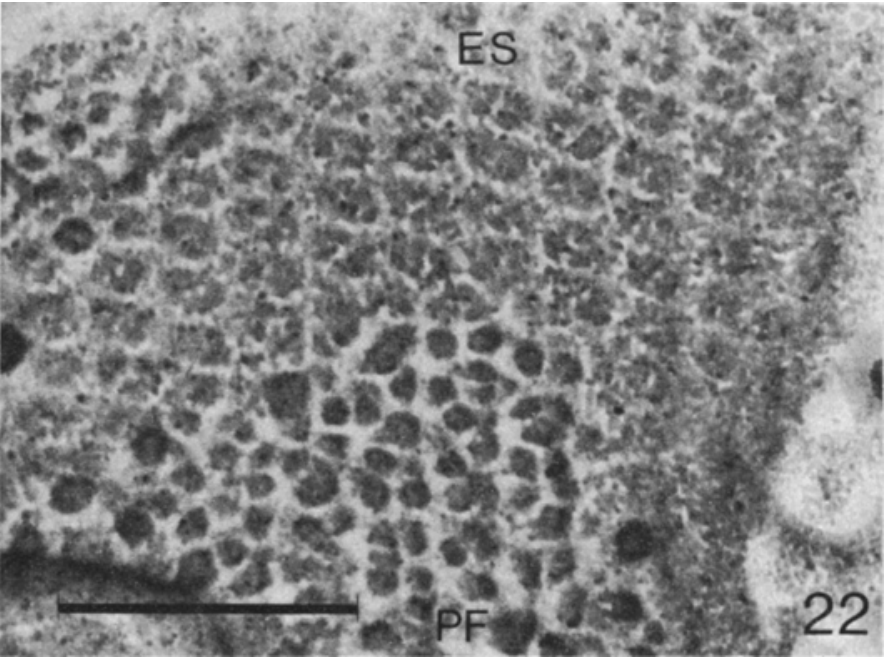

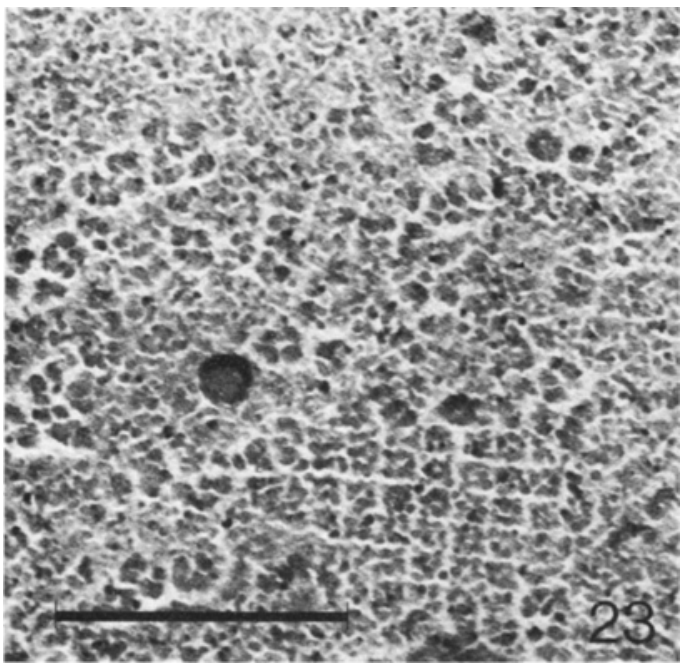

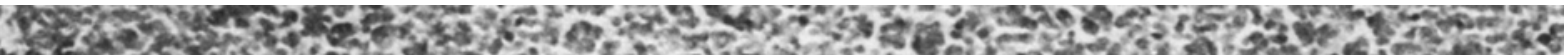

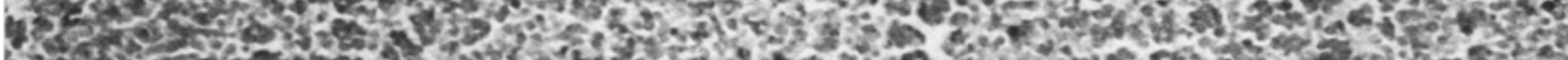

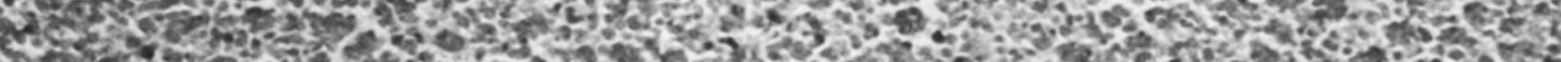

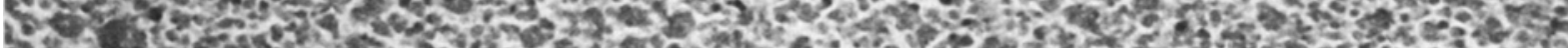

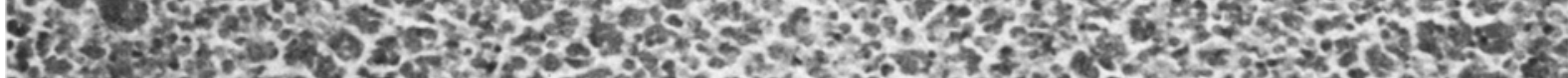

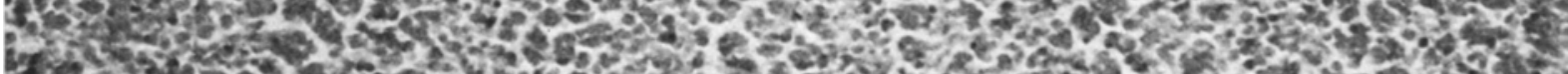

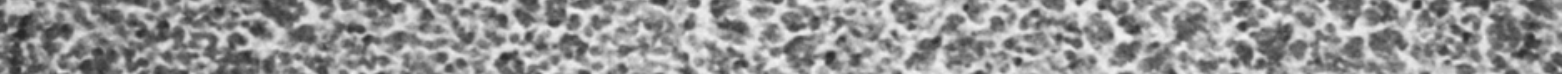

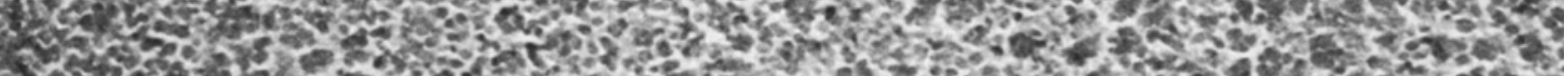

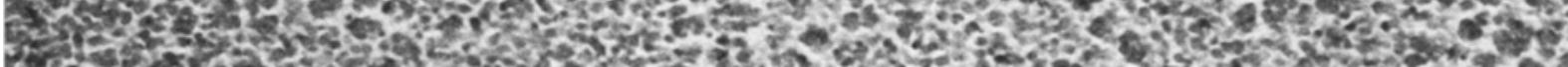

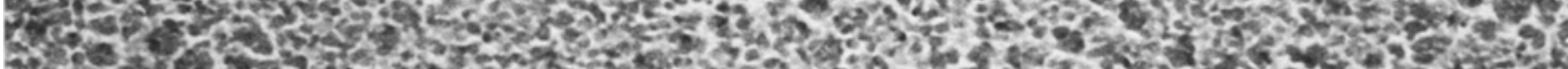

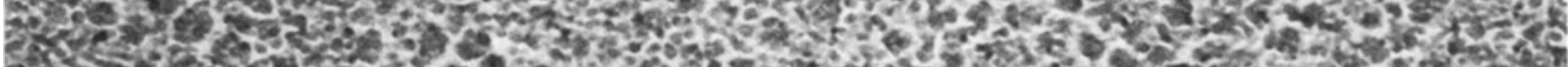

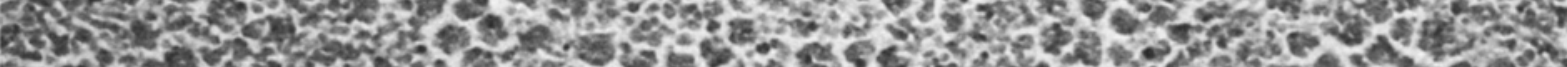

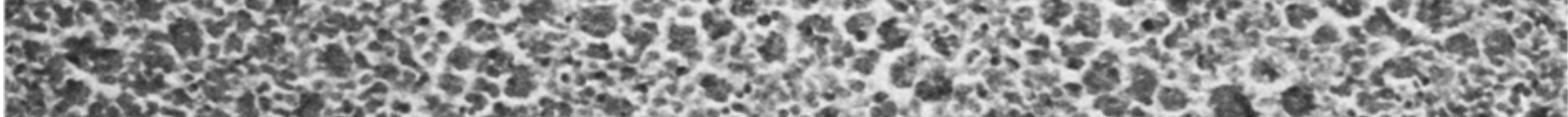

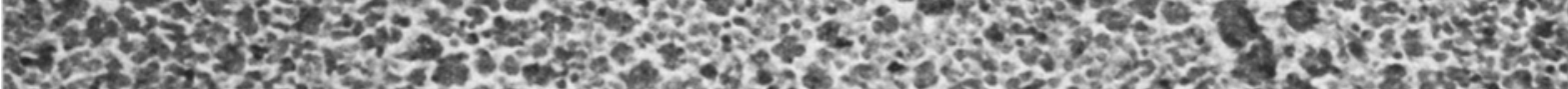

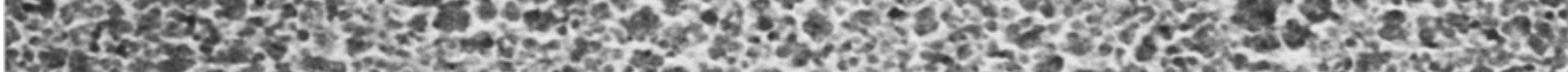

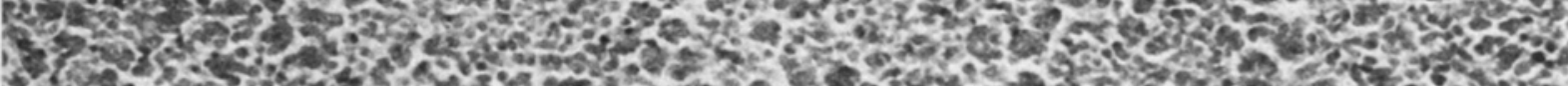

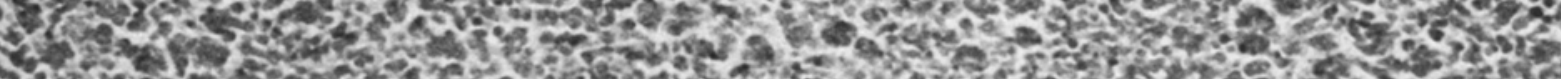

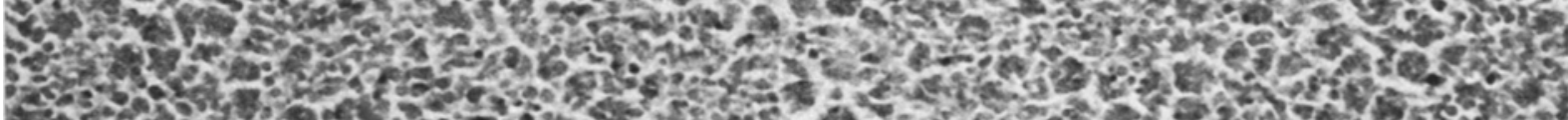

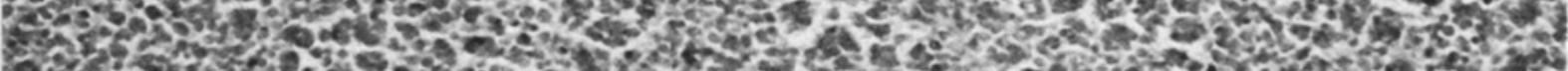

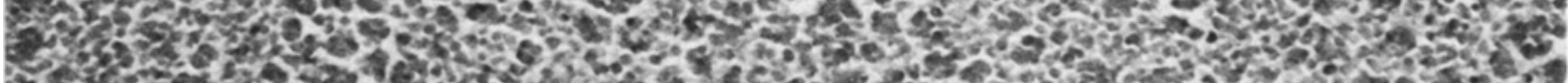

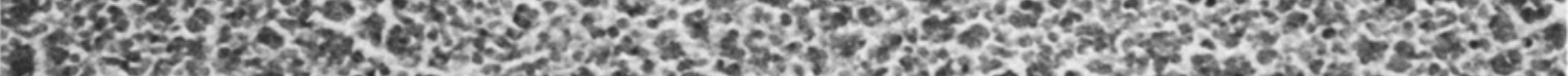

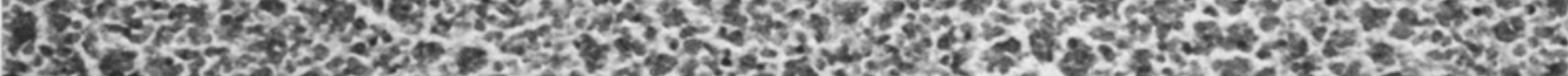

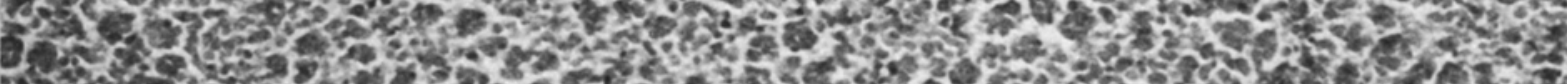

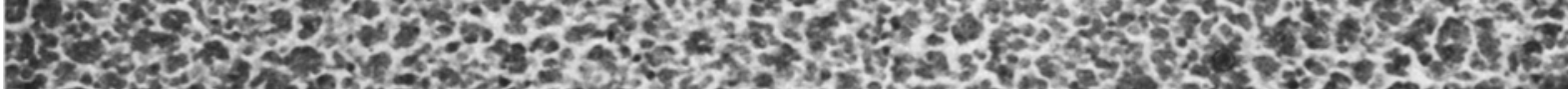

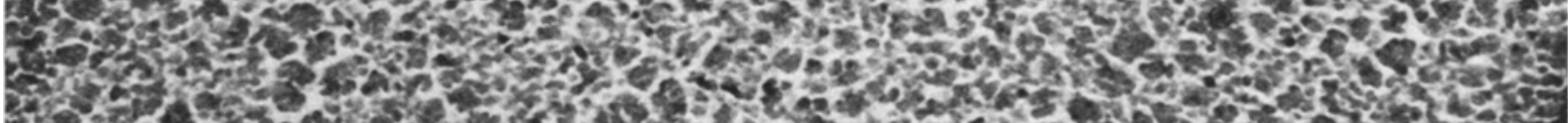

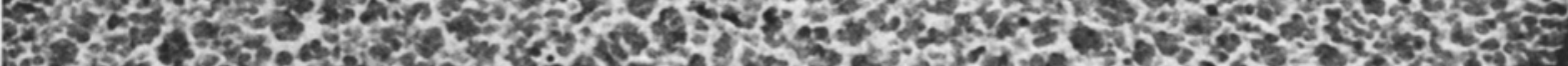

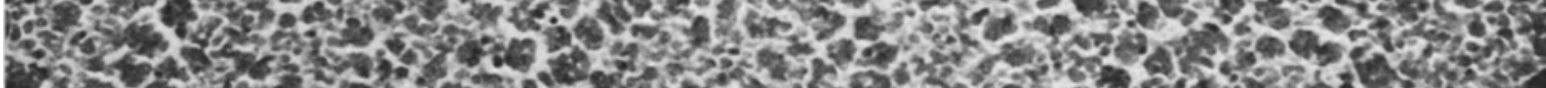

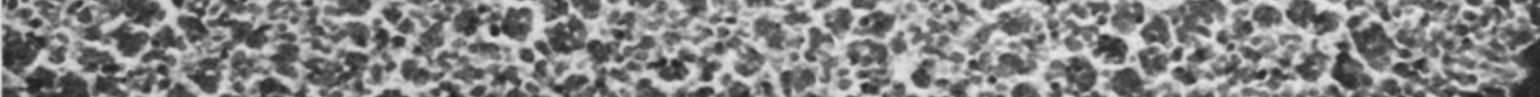

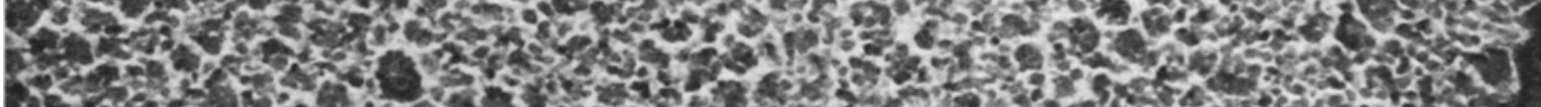

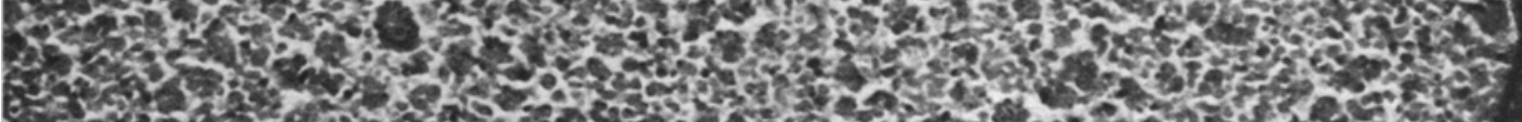

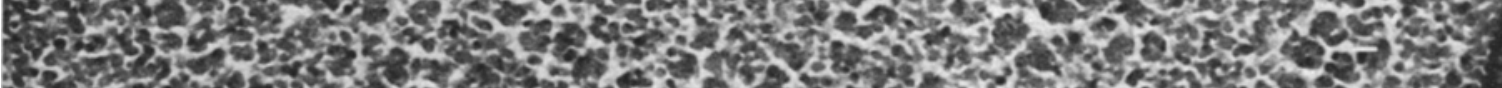

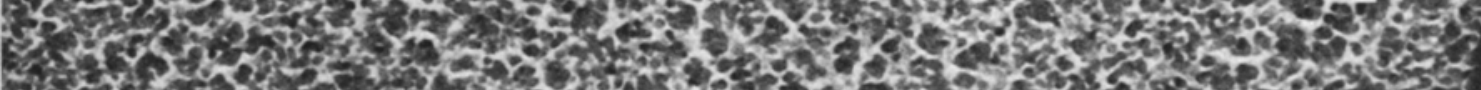

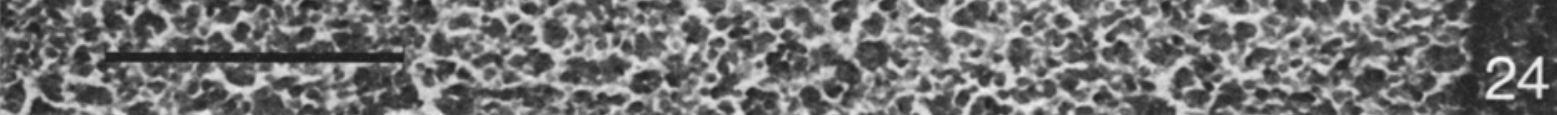


WE

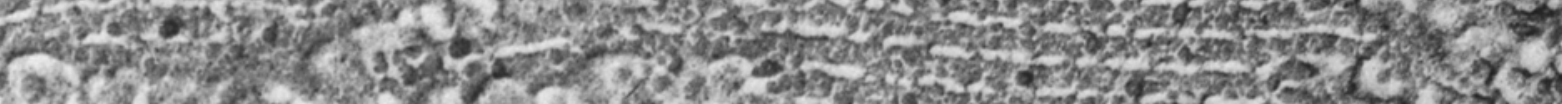

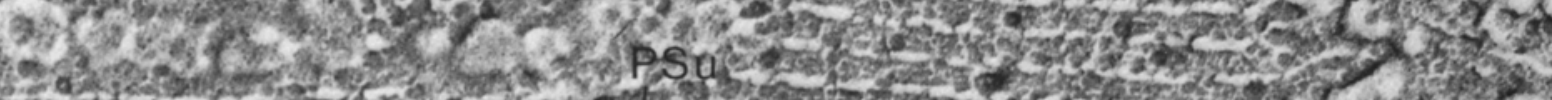

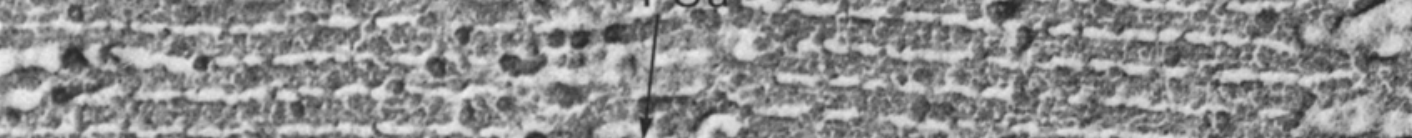

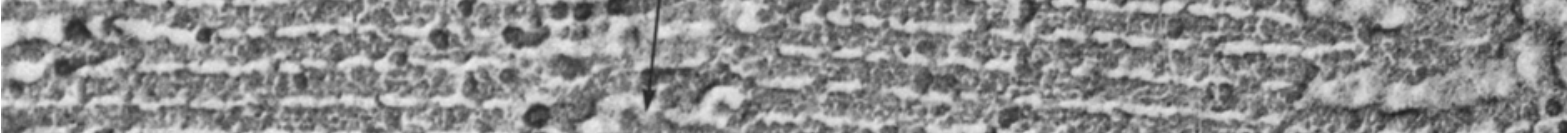

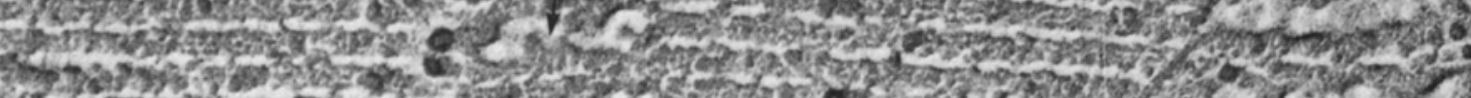

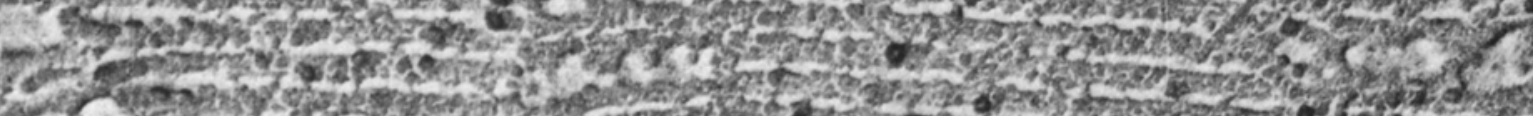

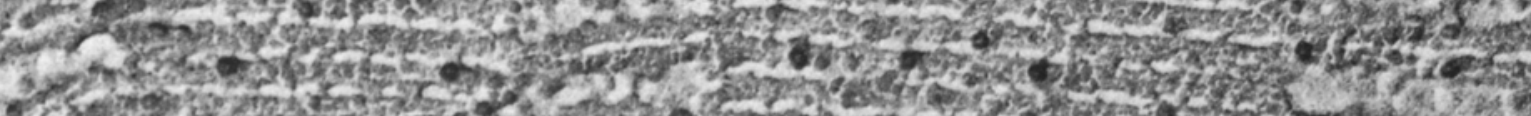

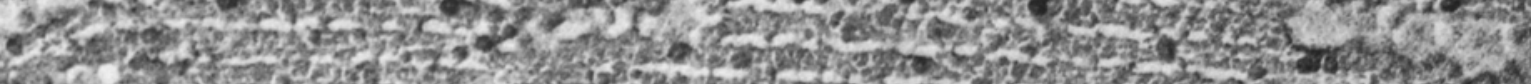

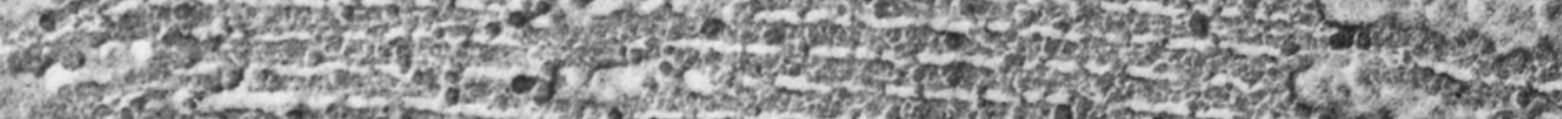

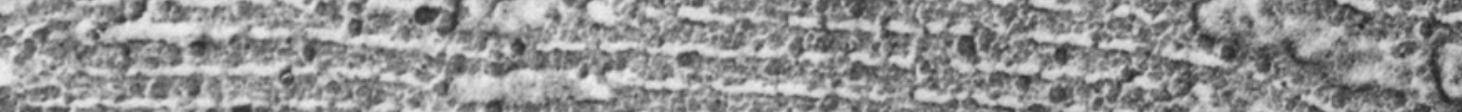

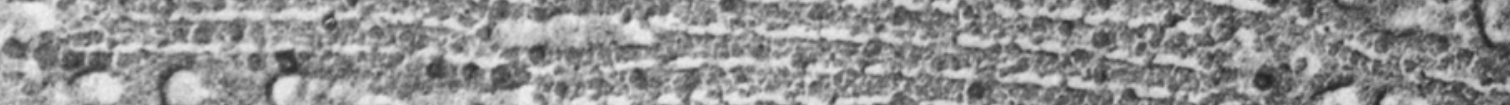

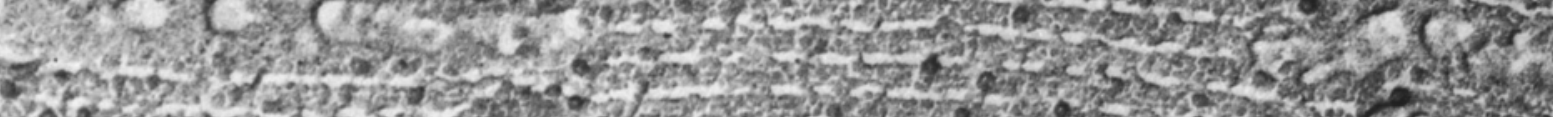

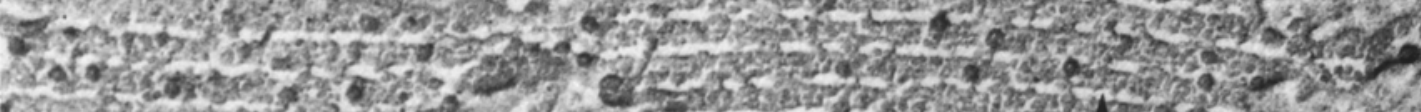

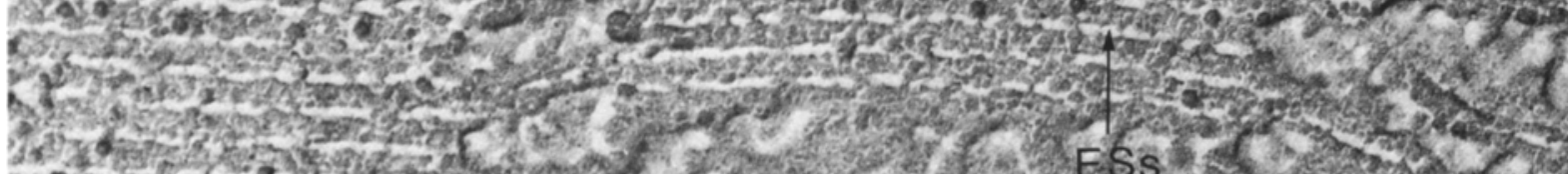

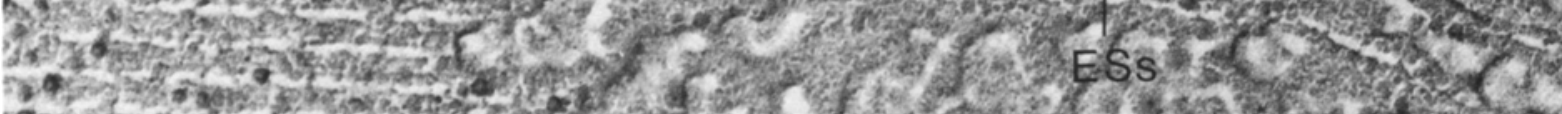

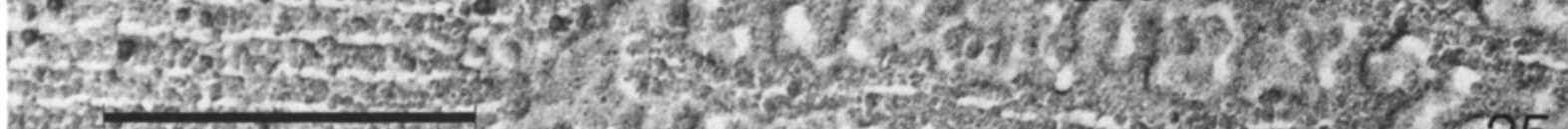

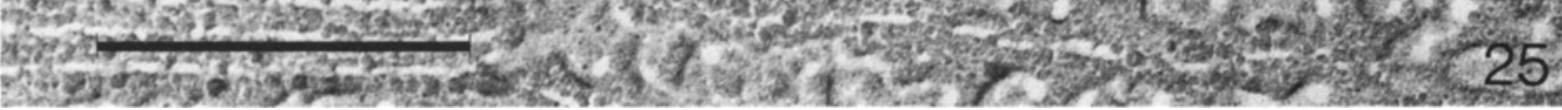

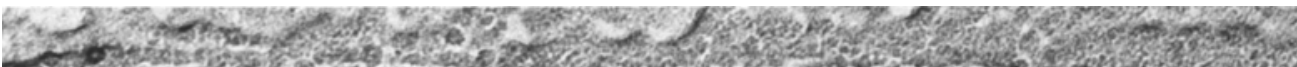

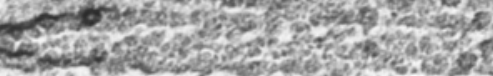

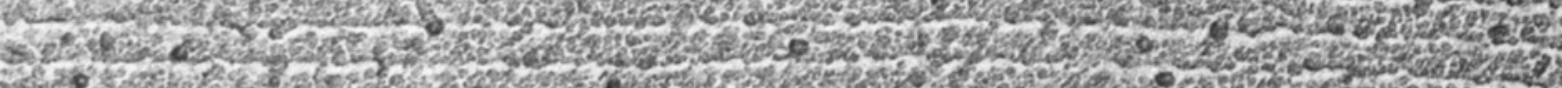

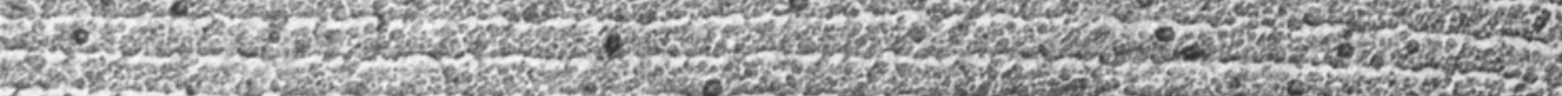

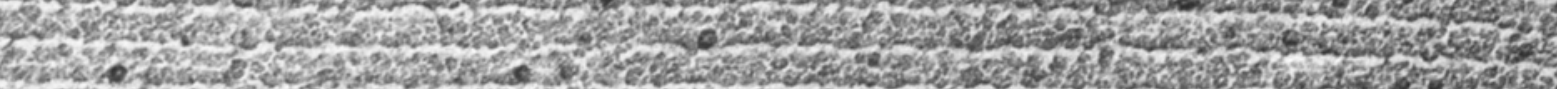

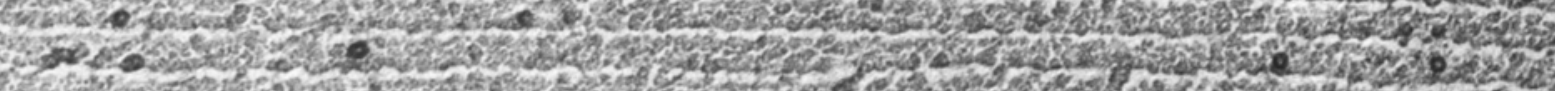

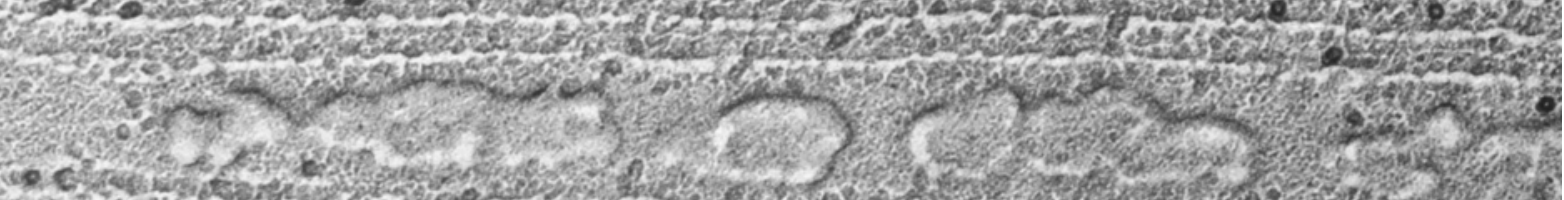

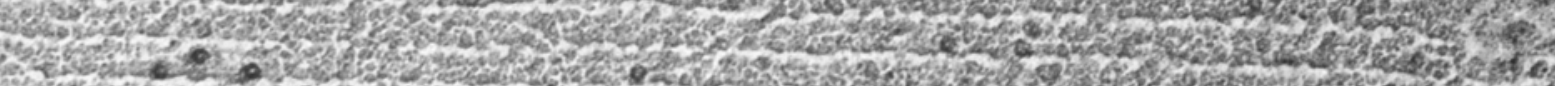

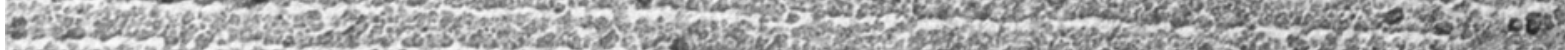

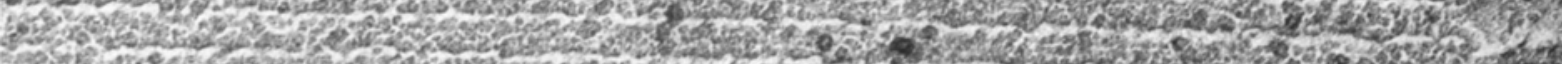

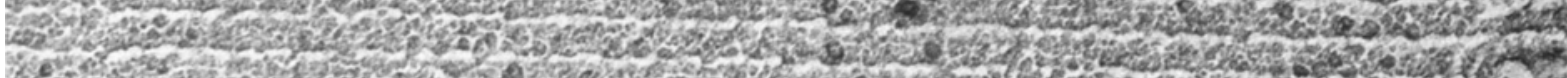

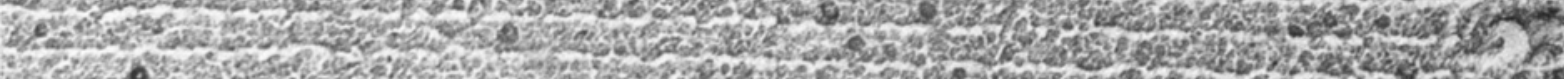

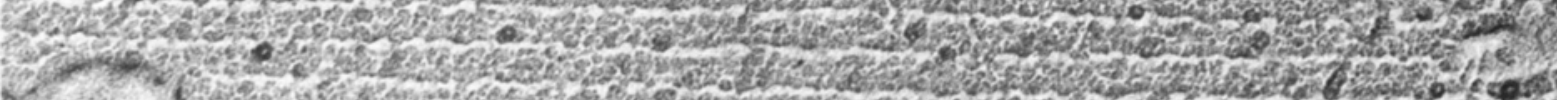

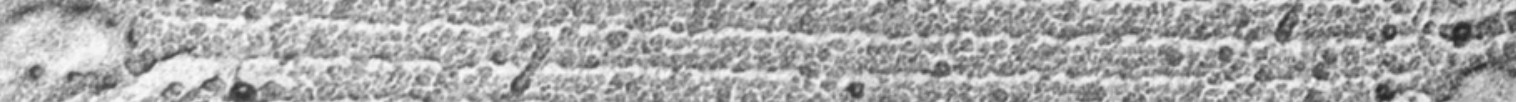

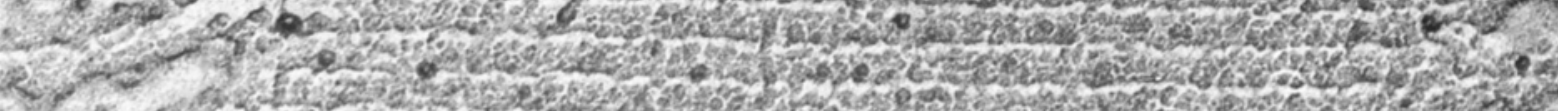

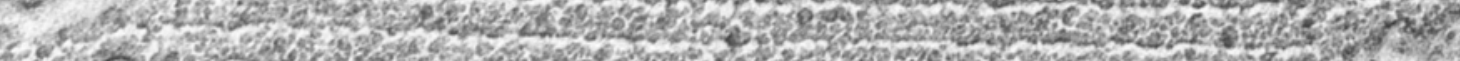
6. 
Figure 25. Rotary shadowed transversely fractured grana of wild-type thylakoids.

This type of micrograph was used to determine the centre-to-centre separation of thylakoid discs, as well as the width of the partition and intrathylakoidal space. The electron dense particles, which are confined to one membrane only in appressed regions, may be plastically deformed freeze-fracture particles. It is possible that the surface irregularities, particularly of the unstacked membranes, are due to particles seen on the PSu and ESs. $\times 150,000$ (Bar $=$ $0.25 \mu \mathrm{m}$ ).

Figure 26. As in Figure 25, showing rotary shadowed transversely fractured grana from chlorina $f 2$ chloroplasts. $\times 150,000(B a r=0.25 \mu \mathrm{m})$.

thylakoidal space (Figures 25 and 26). It was also possible, by looking along the partition, to distinguish the boundary between the two thylakoid membranes. Such micrographs were used to make measurements of the centre-tocentre spacing of the thylakoids in grana for the purpose of comparing wild-type and mutant, and thin-sectioned and freeze-fractured material (Table VII). In addition, the width of the partition and intrathylakoid space in freezefractured material was measured. It was apparent that the thylakoids were much closer together in thin-sectioned material, mainly due to a reduction of the partition width. There was no significant difference, however, in the spacings of freeze-fractured wild-type or chlorina $-f 2$ grana and values for membrane thickness, calculated from the partition width, was about $100 \AA$ in both cases (Table VII). The particles seen in these cross-fractured membranes (Figures 25 and 26) were usually confined to one half of the partition, and never extended across the entire width. These particles could, therefore, be EFs or PFs particles that have been fractured

Figure 27. The polypeptide composition of wild-type and chlorina-f2 thylakoids compared with that of isolated light-harvesting complex.

The polypeptides were separated by SDS-ureapolyacrylamide gel electrophoresis (25). The major difference between wild-type and chlorina- $f 2$ is the absence of the band in the mutant pattern corresponding to the apoprotein of chlorophyll $a / b$-protein 2.

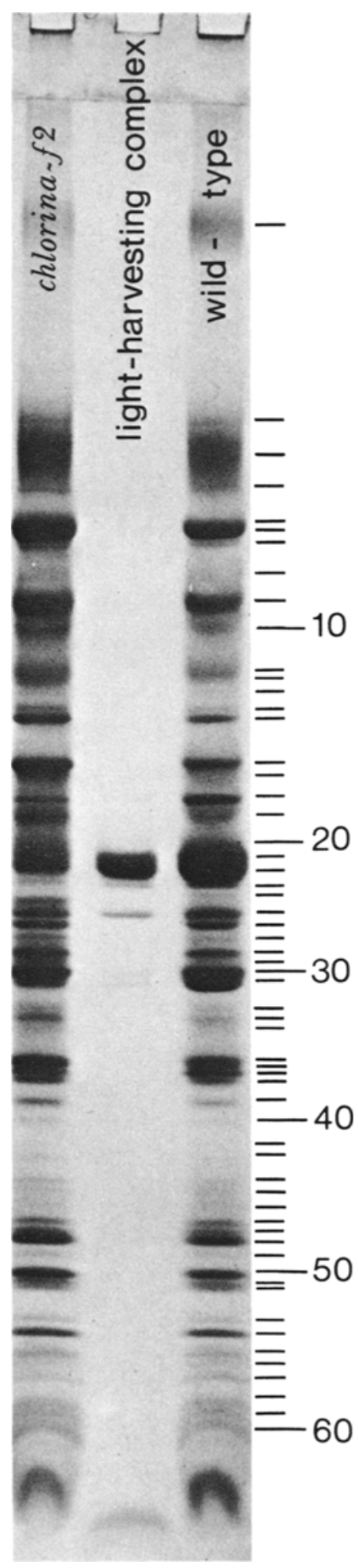




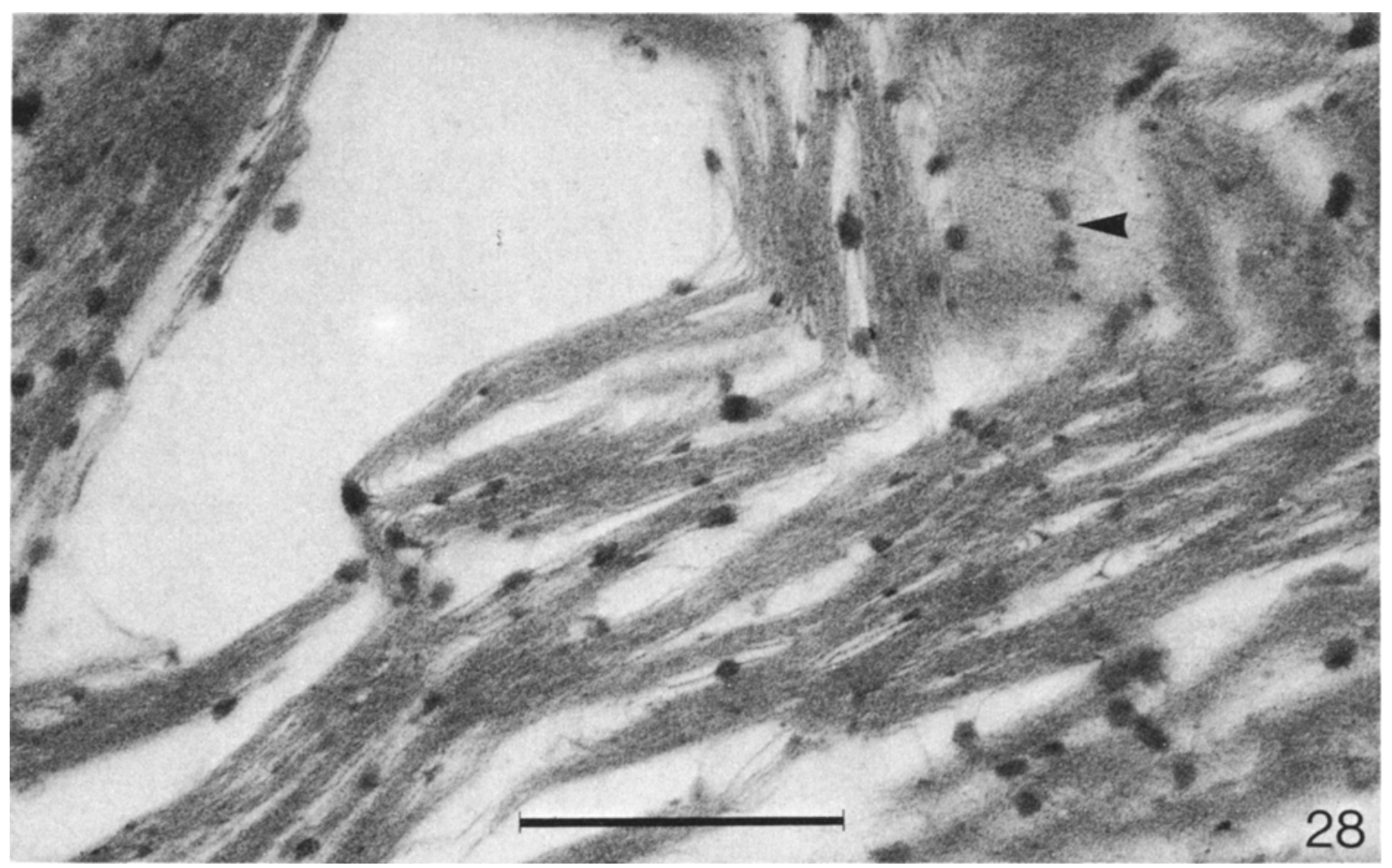

Figure 28. The structure of the isolated light-harvesting complex after $\mathrm{Mg}^{++}$precipitation is shown by conventional fixation and thin-sectioning techniques.

In transverse section, this material has a lamellar structure and forms closely appressed stacks. When sectioned parallel to the plane of the lamella, regions of hexagonally packed particles are revealed (arrowhead). $\times 75,000(\mathrm{Bar}=0.5 \mu \mathrm{m})$.

in a plane at right angles to the plane of membrane cleavage.

A comparison of the polypeptide composition of purified thylakoids isolated from wild-type and chlorina- $f 2$ chloroplasts (Figure 27) shows that the major difference is the absence of the light-harvesting chlorophyll $a / b$-protein (25). It is possible to isolate this protein in reasonably pure form by detergent treatment of wild-type thylakoids (9). Characterisation of the lightharvesting complex by freeze-fracturing and rotary shadowing should be useful to establish which particles they might correspond to in wild-type thylakoids, and to see if such particles are absent from chlorina- $f 2$ thylakoids. The polypeptide composition of purified light-harvesting complex is shown in Figure 27. The final step in the purification of this light-harvesting complex (LHC) involved $\mathrm{Mg}^{++}$precipitation and centrifugation through a $0.5 \mathrm{~m}$-sucrose cushion. The resulting pellet consisted of compact material at the bottom of the centrifuge tube with

Figure 29. The appearance of the light-harvesting complex preparation in Figure 28 is shown after freezefracturing and freeze-etching for $1 \mathrm{~min}$ at $-100^{\circ} \mathrm{C}$, followed by rotary shadowing.

The fracture face contains hexagonally arrayed particles with an average centre-to-centre distance of $122 \mathrm{~A}$. The etched surface consists of hexagonally arrayed electron transparent holes. The rows of holes on this surface coincide with the grooves between rows of particles on the fracture face. The boundary between the fracture face and etched surface is easily recognised (arrowheads). $\times 300,000(\mathrm{Bar}=0.1 \mu \mathrm{m})$.

Figure 30. Light-harvesting complex vesicles (liposomes) from the soft pellet, showing areas of hexagonally arrayed particles and areas of lipid containing individual particles (arrows).

Particle arrays were seen with equal frequency on both convex and concave (asterisk) faces. $\times 100,000 \mathrm{Bar}=$ $0.5 \mu \mathrm{m})$. 


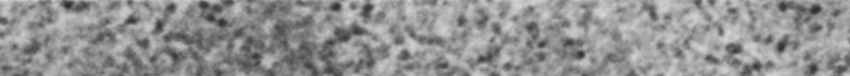

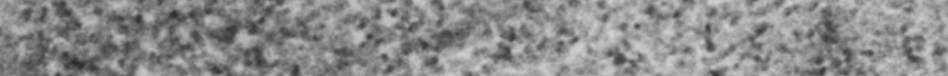

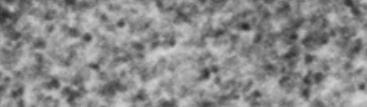

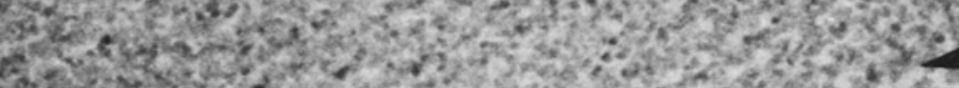

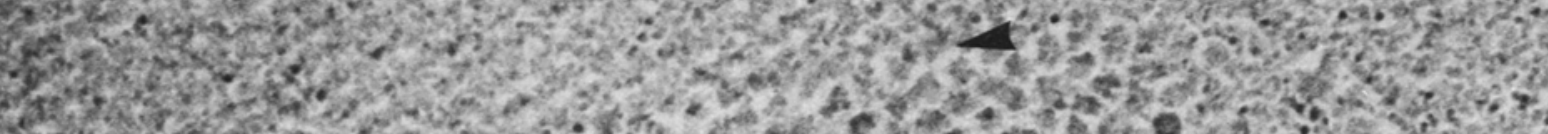

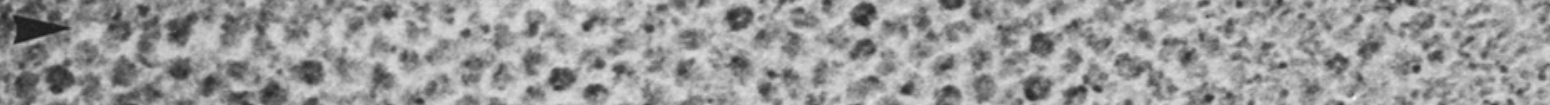

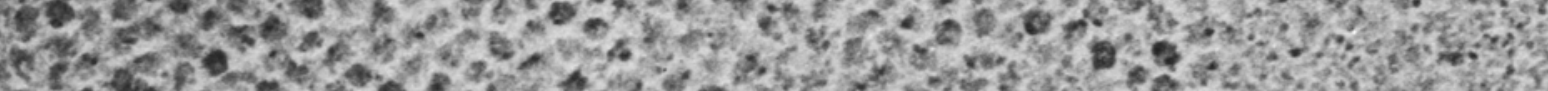

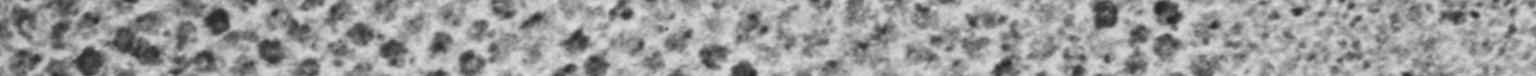

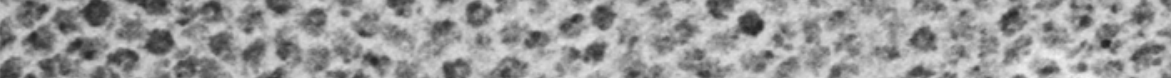

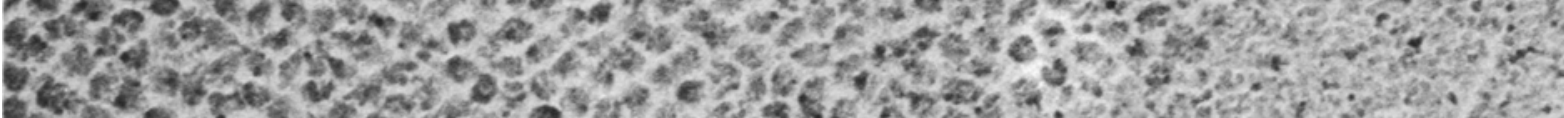

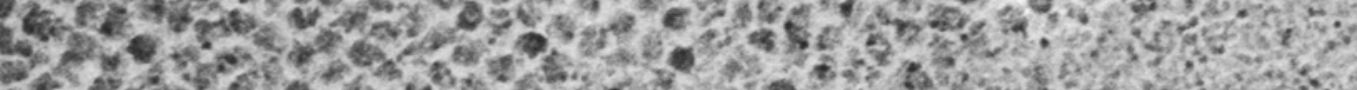

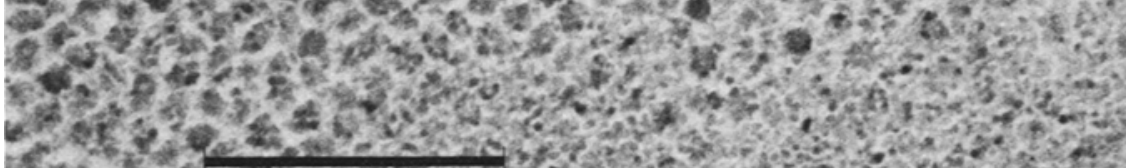

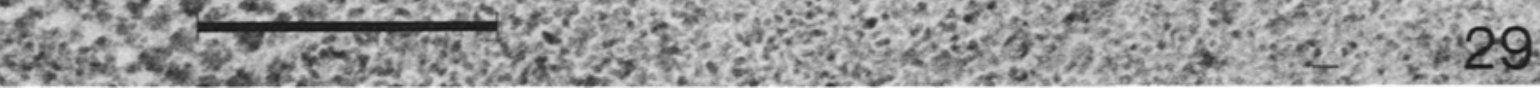

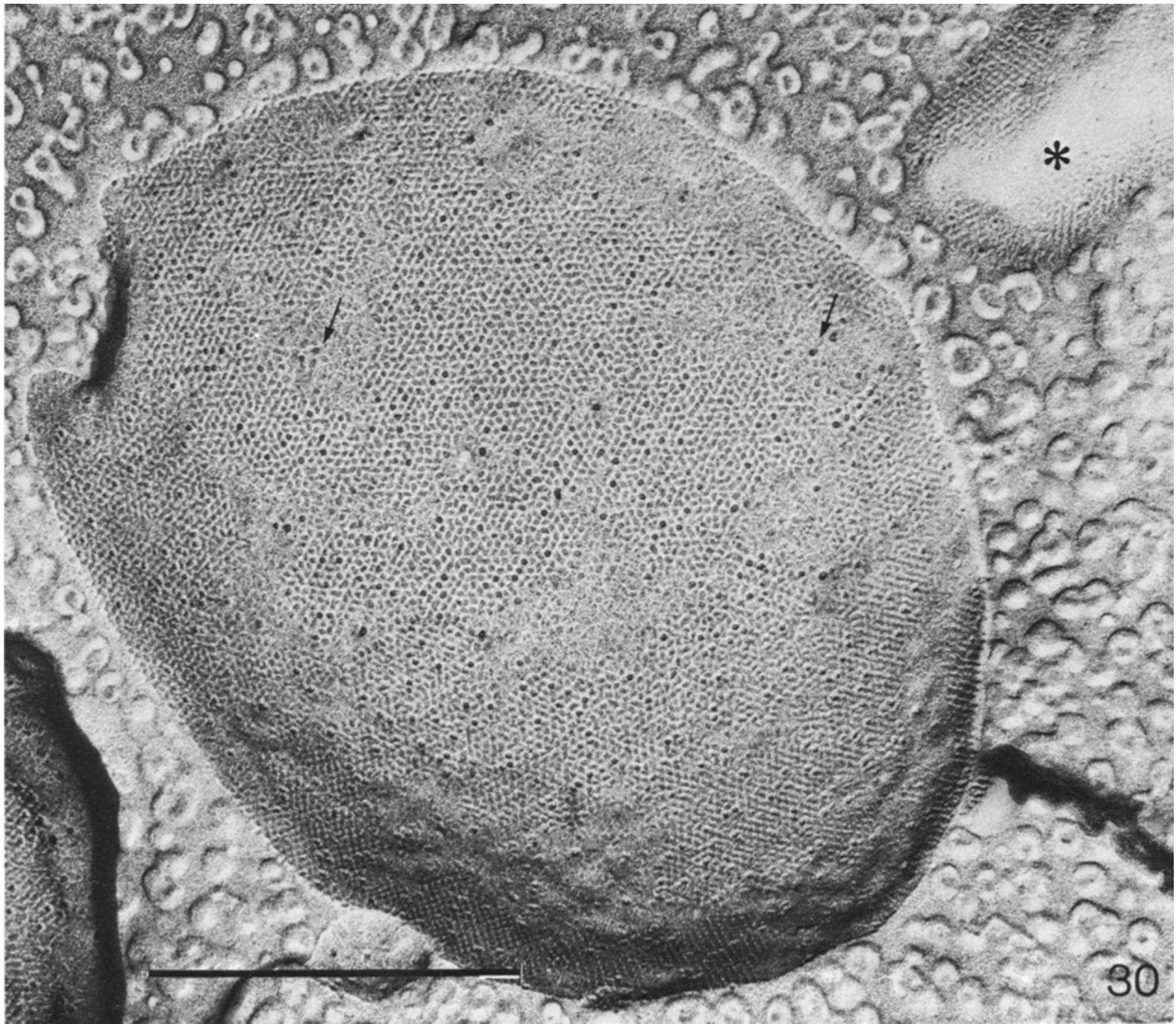


softer material above it. The appearance of the hard pellet after fixation and thin sectioning is shown in Figure 28. It consisted of membranelike lamellae which appeared to stack together in many places. When these lamellae were sectioned parallel to their surface, a hexagonal lattice was seen (Figure 28). The same material was examined by freeze-fracturing and rotary shadowing and it was found that the lamellae directed the fracture plane, revealing particles in hexagonal arrays (Figure 29). It can be seen that these lamellae were cleaved by freeze-fracturing since there is a discontinuity (Figure 28) where the fracture face meets the etched surface. The rows of particles on the fracture face coincided with the lines between rows of holes in hexagonal arrays on the etched surface.

The soft pellet contained numerous large vesicles which were visible under phase contrast light microscopy. When freeze-fractured, these vesicles contained particles in close-packed arrays and less densely packed particles in lipid rich areas (Figure 30 ). These arrays deviated by no more than $2^{\circ}$ from a hexagonal lattice and the average centre-to-centre spacing in all three directions was $122.3 \pm 1.9 \AA$ ( \pm S.D.). It can be seen from Figures 29 and 30 that there were two types of particles. The majority was of moderate electron density and had dimensions of $70.3 \pm 7.3$ by $94.6 \pm 11.0 \AA$ ( \pm S.D.). The other type of particle was very electron dense with more sharply defined edges and had an average size of $77.4 \pm 6.3$ by $87.4 \pm 6.7 \AA$ ( \pm S. D.). These particles were found both in arrays and in smooth areas by themselves. Particle arrays covered most of the area of the vesicles and were found on both convex fracture faces and concave fracture faces (Figure 30 ). The size of the particles in freeze-fractured light-harvesting complex vesicles correspond closely with that of the particles missing from the PFs face of chlorina- $f 2$ thylakoids.

\section{DISCUSSION}

Rotary shadowing, particularly of the EFs face, has shown that freeze-fracture particles of thylakoids range in shape from circular to elliptical, i.e., they have this cross-sectional shape in the plane of membrane cleavage. This confirms earlier work (35) predicting elliptical shape of EFs particles in ordered arrays. The variety of particle sizes and shapes explains why it is not usually possible to resolve EFs particles into discrete size classes when their size distribution is plotted (Figure 17). Even when measurements are made on a population of particles known to have the same composition and size (those of the light-harvesting complex), the standard deviation of such measurements is about $6.5 \AA$. To determine the precision of the digitiser, one hundred figure 0 's on a sheet of Letraset were measured. These $7.7 \mathrm{~mm}$ high figures were imagined to be 800,000 times magnified and yielded values of $98.4 \pm 1.5 \AA$ by $72.0 \pm 2.4 \AA$ ( \pm S.D.), for the large and small diameters, respectively. Thus, the method is sufficiently accurate to resolve populations of uniformly sized freeze-fracture particles differing in size by $13 \AA$ (i.e. 2 times $6.5 \AA$ ).

The technique of rotary shadowing of etched surfaces enables the visualisation of surface particles with greater clarity than is possible with uni-directional shadowing. This is due partly to the lower shadowing angle $\left(22.5^{\circ}\right.$ versus $\left.45^{\circ}\right)$ and partly to the fact that the particles are coated on all sides, so that detail is not obscured by the shadow. The tetrameric substructure of the ESs particles is particularly clear, and fine structure of the ESu has been revealed in greater detail than before. There are also suggestions of substructure in the large PSu particles (Figure 24), although not as clearly and uniformly as for ESs particles. Ribulose-1,5-bisphosphate carboxylase and coupling factor 1 are located on the $\mathrm{PSu}$ (39) and contain subunits with molecular weights of about 55,000 daltons (45).

No attempt was made in this study to describe fine structure of rotary shadowed particles on fracture faces. It seems likely that any such detail is destroyed by plastic deformation during freeze-fracturing of thylakoids, although it is thought that this deformation is less extensive in membranes where complementarity between the EF and PF faces is preserved $(14,22)$. Such fine structure has been reported for rotary shadowed particles of the PF face of the red blood cell membrane (26), but it is not clear to what extent it is caused by decoration, particularly under conditions of low shadow angle and a thin coating of platinum. 

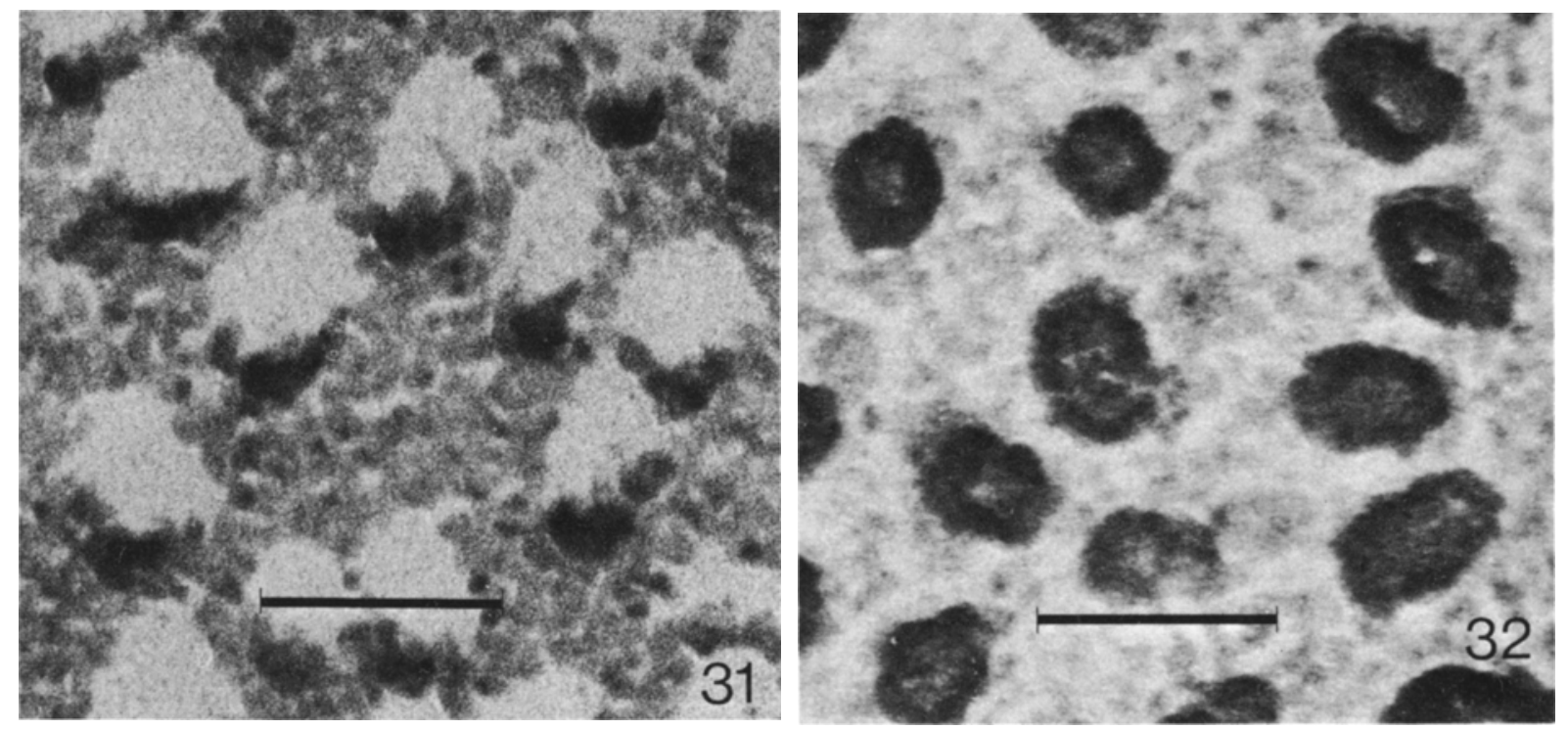

Figure 31. High magnification electron micrograph of the particles on the EFs face of chlorina- $f 2$ thylakoids after freeze-fracturing and uni-directional shadowing at $45^{\circ}$.

The shape of the shadow indicates that the particles have a rounded contour and are probably hemispherical, although judging from the length of the shadow ( = particle height $=150 . \AA$ ), considerable plastic deformation must have occurred during freeze-fracturing. $\times 1,000,000($ Bar $=25 \mathrm{~nm})$.

Figure 32. Rotary shadowed EFs particles of chlorina $f 2$ thylakoids.

Particle shape varies continuously between circular and elliptical, and each particle has the appearance shown schematically in Figure 2. $\times 1,000,000($ Bar $=25 \mathrm{~nm})$.

It is often difficult to make accurate measurements of particle density on densely populated fracture faces from uni-directionally shadowed replicas. Particles may not be visualised if they are of low surface relief and/or lie in the shadow of larger particles. Rotary shadowing overcomes this problem to some extent as demonstrated by the increased density found on the PFs face of rotary shadowed wild-type thylakoids compared with uni-directionally shadowed material (Tables II and III). There is still a degree of subjectivity in deciding whether a feature is a particle for the purposes of counting. The lower shadowing angle used in rotary shadowing allows the holes in the EFu face of the thylakoid to be visualised easily (compare Figures 13 and 14). The density of these holes was estimated to be $3298 \pm 46$ ( \pm S.E.), which is about two-thirds of the density of the particles on the $\mathrm{PFu}$ face. It remains to be determined whether all particles on the PFu face have complementary holes, or only a fraction of them.

There is a lower limit to the shadowing angle that can be usefully employed during rotary shadowing. In particular, shading of one particle by others becomes more and more pronounced as the angle is decreased. Although it is not possible to make accurate estimates of particle heights from uni-directionally shadowed replicas, since it is difficult to determine the angle of the fracture plane with respect to the evaporation source, an approximate value of $150 \AA$ seems reasonable for EFs particles (see Figure 31). The shadow length is equal to the particle height divided by the sine of the shadowing angle, which for EFs particles shadowed at $22.5^{\circ}$ is $390 \AA$. Particles on the EFs face are closer together than $390 \AA$ so that the background of the EFs face is less electron dense than the EFu (Figure 7) due to a thinner layer of platinum 
resulting from shading by overlapping shadows of EFs particles.

When the plane of an area of a rotary shadowed specimen deviates from the horizontal, the particles cast a shadow on one side, and have much less platinum deposited on that side (see areas in Figures 24 and 30). It is therefore easy to determine visually whether a surface is horizontal or not. The values obtained from rotary shadowed replicas for the particle densities of EFs and EFu faces are slightly lower than corresponding values from uni-directionally shadowed material (Tables II and III). The probable explanation is that areas used from uni-directionally shadowed thylakoids were not always horizontal, causing a reduction in the size of the projected area and an apparent increase in measured particle density. One can use shadow length as a criterion for tilting in the direction of the evaporation source, and focusing differences to determine tilting of the specimen at right angles to the evaporation gun, but an error of only $10^{\circ}$ in both directions, causes a $3 \%$ overestimation of particle density.

Visual selection of transversely fractured grana oriented horizontally in rotary shadowed material allows the accurate determination of the repeat distance between thylakoid discs and the width of the thylakoid membrane in material that has not been subjected to chemical fixation, dehydration, embedding and thin-sectioning. The value of approximately $270 \AA$ for the repeat distance and $200 \AA$ for the partition thickness is consistent with earlier freeze-fracture results, and values measured from aqueous-plasticembedded material (see 21). Since the lipid bilayer is $40 \AA$ thick, or maximally $60 \AA$ in freeze-fractured membranes (21), and a grana thylakoid membrane is $100 \AA$ thick, the protein projects at least $40 \AA$ out of the bilayer. Further experiments are needed to decide if the particles seen in transversely fractured membranes correspond to EFs and PFs particles fractured at right angles to the normal freeze-fracture plane (Figures 25 and 26).

A comparison of particle sizes in corresponding faces of uni-directionally and rotary shadowed thylakoids revealed surprisingly small differences (Tables IV and V). In the case of chlorina $-f 2$ thylakoids and for the EFu face of wildtype, these comparisons were made from rotary shadowed and uni-directionally shadowed replicas prepared from the same material. Sizes obtained for rotary shadowed material were about $7 \%$ greater than uni-directionally shadowed thylakoids, except for the EFs face, where particles were about $5 \%$ smaller in rotary shadowed replicas. It had been assumed that determination of shadow width at the base of the particle at right angles to the direction of shadowing in uni-directionally shadowed material would provide an accurate measure of particle width (39). Calculations for rotary shadowed particles (Section 2.4) indicated that the size of rotary shadowed particles would be overestimated by about $30 \AA$. These calculations were based upon the assumption that, among other things, particles were approximately hemispherical after freeze-fracturing. An examination of the shape of the shadow of unidirectionally shadowed chlorina- $f 2$ EFs particles (Figure 31) indicates that this assumption is justified, and the appearance of these particles after rotary shadowing (Figure 32) is consistant with that predicted for a hemispherical particle (Figure 2). It is possible that shading effects may cause a reduction in the thickness of platinum deposited at the base of a particle, particularly on the PFu and PFs faces. This phenomenon may be responsible for the slight reduction in width of ESs particles in arrays (see Table VII). An investigation of platinum-shadowed gold particles indicated that measurement of shadow width of uni-directionally shadowed particles overestimates the true diameter by between 40 and 70 $\AA$, depending on particle size (33). This was due to growth of platinum crystals on the gold particle. The present study shows that unidirectionally shadowed freeze-fracture particles have the same diameter as the corresponding rotary shadowed ones. This suggests that values for particle widths from uni-directionally shadowed replicas may be overestimated by about 30 $\AA$ on faces that are not densely populated. On the other hand, the size of the large particles on the PSu $(100 \times 120 \AA)$ is close to the reported values for coupling factor 1 , which is a sphere with a diameter of $110 \AA$ (31) and ribulose-1,5bisphosphate carboxylase, which is a cube with $120 \AA$ long sides (46). The relationship between particle size as measured from freeze-etched preparations and by physical methods needs to 


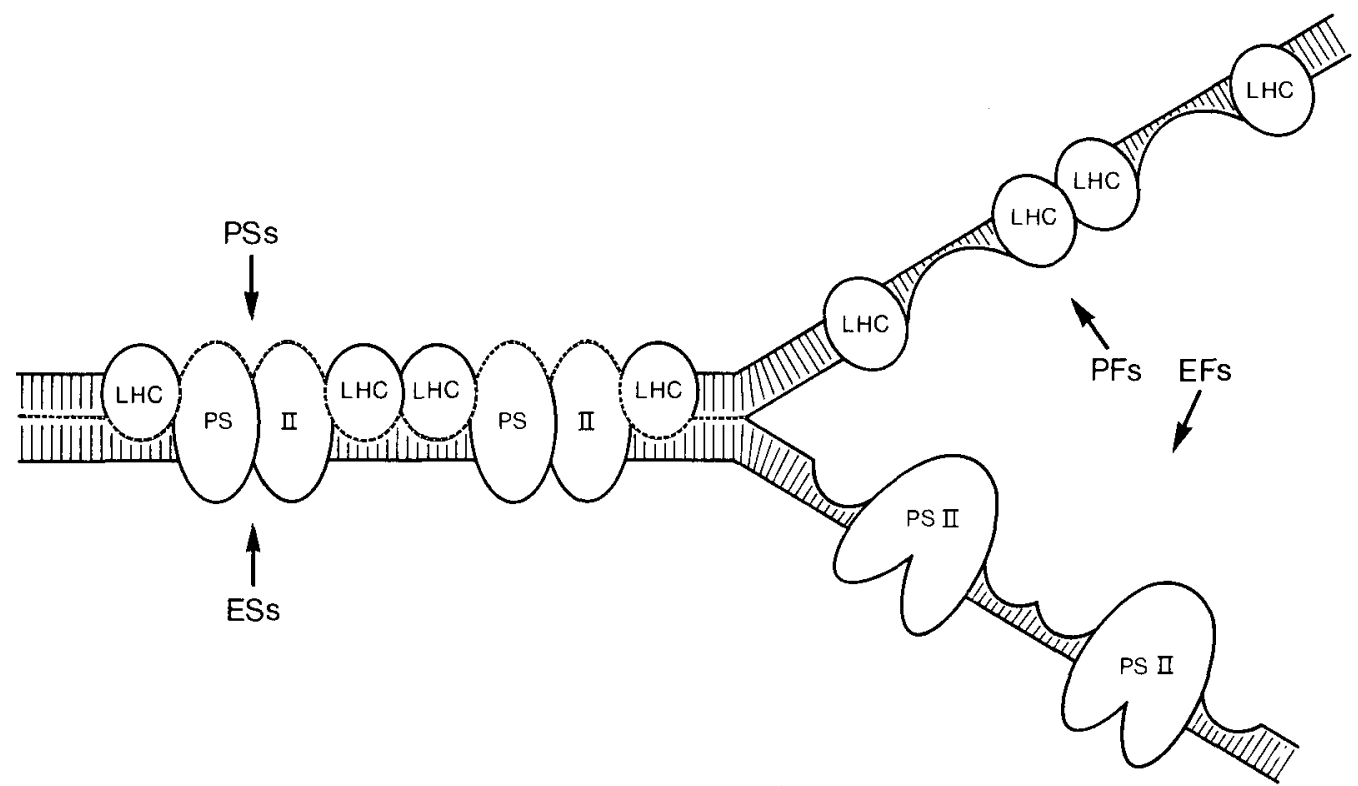

Figure 33. Diagrammatic representation of the freeze-fracture behaviour of the thylakoid membrane from grana of wild-type barley chloroplasts.

The light-harvesting complex is visualised as being localised in particles distinct from the EFs particles, but closely associated with them. During freeze-fracturing the light-harvesting complex and EFs particles are separated by the fracture plane and cleave with opposite halves of the thylakoid membrane.

be determined using lipid bilayers into which a single purified protein of known dimensions has been inserted.

The chlorina- $f$ mutant lacks chlorophyll $b$ and consequently has no chlorophyll $b$-containing proteins $(6,25)$. Although the original paper describing the ultrastructure of the chloroplasts of this mutant reported the presence of grana (16), many authors cite this mutant as an instance where the absence of the light-harvesting chlorophyll $a / b$-protein is correlated with the absence of grana. This seems to have resulted from a desire to establish a causal relationship between light-harvesting chlorophyll $a / b$ protein and stacking in thylakoids, since early studies of detergent fractionated thylakoids indicated that the light-harvesting chlorophyll $a / b$-protein was located in the grana (see ANDERSON for a review). It has been shown by others $(10,16,30)$ and by the present investigation using plants of the same age and grown under the same conditions as the wild-type control, that grana are present in chlorina-f2 (Figure 5). In seven-day old seedlings, most of the grana in chlorina- $f 2$ chloroplasts are at least twice the diameter of wild-type grana, and they have a slightly lower number of discs (Table VII).

The striking difference between the freezefracture ultrastructure of wild-type and chlorina$f 2$ thylakoids is in the appearance of the PFs face, both in uni-directionally and rotary shadowed preparations (Figures 11 and 12). In the latter there is a reduction in the number of particles from 6257 to 1124 per $\mu^{2}$ (Table II). It is concluded that since chlorophyll $a / b$-protein 2 is such a major polypeptide in wild-type thylakoids, it is located in the PFs particles absent from the chlorina- $f 2$ mutant (Fig. 33). It is known that PFs particles align themselves with respect to EFs particles when such particles form arrays $(35,38)$, and are probably in intimate contact with them. The major difference between the interpretation in Figure 33 and that of ARMOND et al. (3) is that the freeze-fracture plane follows a hydrophobic region between the lightharvesting complex and the EFs particle so that each cleaves with opposite sides of the mem- 
brane. This is consistent with the method for isolating the light harvesting complex in which Triton X-100 is used to disrupt the hydrophobic association between this protein and the other membrane components (9). Furthermore, the size of the freeze-fracture particles in lightharvesting complex vesicles is close to that of the particles missing from the chlorina- $f 2$ PFs face, but present on the wild-type PFs face.

It is likely that the EF particles of thylakoids are the structural equivalents of photosystem II units, although the evidence is indirect $(3,4,40)$. Since fluorescence studies indicate a close association between the light-harvesting chlorophyll $a / b$-protein and photosystem II reaction centres $(4,23)$, many models of the structure of the thylakoid membrane place the light-harvesting chlorophyll $a / b$-protein in the EFs particles (3, $4,12,21,40$ ). Most of the evidence to support this model comes from a study of the greening in continuous light of plants grown under intermittent light (3). It was found that EFs particles increased in size in a stepwise manner which was correlated with the appearance of the lightharvesting chlorophyll $a / b$-protein during greening. The major change in the freezefracture ultrastructure during greening of these plants was the increase in EFs particle size, and the major change in the polypeptide composition was an increase in the amount of the lightharvesting chlorophyll $a / b$-protein. This was interpreted as an addition of light-harvesting chlorophyll $a / b$-protein subunits to a photosystem II 'core' particle $(3,18)$. Apart from difficulties in resolving particle size groups in a population of elliptical particles shadowed from one direction, there was an implicit assumption that the PFs particles that appeared during greening were the same as, or were derived from, particles present on the PF face before greening commenced. In addition, it is not clear from scans of the SDS-polyacrylamide gels published, that the light-harvesting chlorophyll $a / b$-protein was the only component which showed a relative increase during greening.

A model in which the light-harvesting chlorophyll $a / b$-protein was located in EFs particles was apparently supported by early freezefracture studies of the light-harvesting chlorophyll $a / b$-protein deficient chlorina- $f 2$ thylakoids which were reported to lack large EFs particles $(1,5)$. These studies were perhaps complicated by the instability of these membranes in low salt conditions. The results from MiLLER et al. (30) and the present paper, clearly demonstrate the presence of EFs particles in chlorina- $f 2$ thylakoids. The SDS-urea polyacrylamide gel in Figure 27 shows that the mutant thylakoids contain almost all of the 60 polypeptides resolved in wild-type thylakoids. For greening of pea thylakoids after intermittent illumination, an EFs particle size increase of $100 \%$ was found (3), which is comparable to the $80 \%$ increase determined for greening of dark-grown barley thylakoids $(34,35)$. In contrast the EFs particles of chlorina $-f 2$, which completely lacks the light-harvesting chlorophyll $a / b$-protein, are only $12 \%$ smaller than wild-type (Table IV).

The diagram in Figure 34 is a hypothetical representation of the organisation of the lightharvesting complex and EFs particles within the grana membrane. The figure is drawn to scale, taking into consideration values obtained for partition thickness, particle spacings in EFs, PFs and light-harvesting complex arrays, and particle sizes at the ESs and fracture plane. This arrangement may offer a mechanism for energy transfer which is known to occur between at least four photosystem II units $(11,19)$. One of the problems in placing photosystem II reaction centres in EF particles is that they appear to be too far apart to allow energy transfer, but if this process can occur across two appressed thylakoids via the light-harvesting chlorophyll $a / b$-protein located in PFs particles, this objection is overcome (see also ARNTZEN (4)). It seems that the light-harvesting chlorophyll $a / b$-protein does not mediate energy transfer within the plane of a single thylakoid membrane, since it does not occur in de-stacked thylakoids, as deduced from the kinetics of fluorescence induction in the presence of 3-(3,4-dichlorophenyl)-1,1-dimethylurea (see 19). No measurements of this kind have been published for stacked thylakoids of chlorina- $f$, so it is not known if energy transfer occurs across the grana in this mutant, although the fluorescence induction kinetics of chlorina- $f 2$ and wild-type barley leaves are qualitatively the same (23). Energy transfer between photosystem II units has been demonstrated in non-stacked membranes, however, in the III-C mutant of Cyanidium calda- 


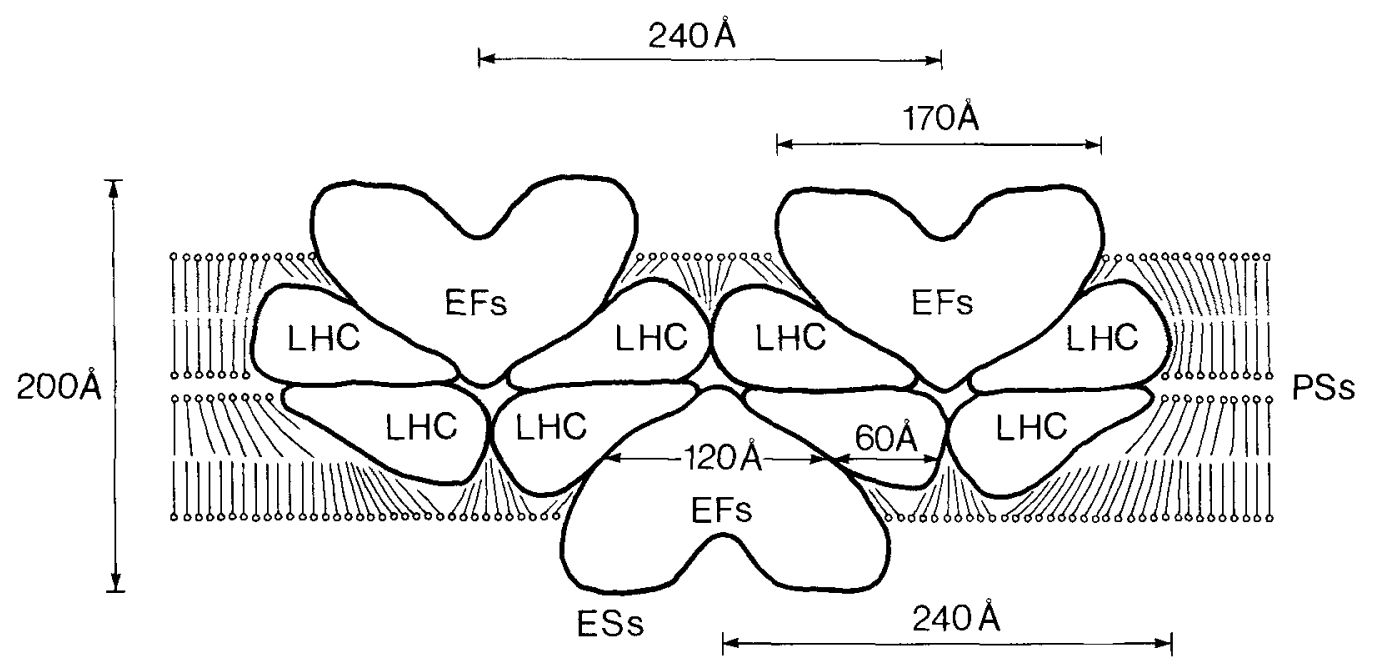

Figure 34. Model of the thylakoid membrane from the grana of wild-type barley chloroplasts.

The size and shape of the EFs and PFs particles have been calculated from measurements of EFs particles and those in light-harvesting complex vesicles, which yield the cross-sectional size at the fracture plane. In addition. the centre-to-centre distance of light-harvesting complex particles in arrays has been used as the maximum width of the PFs particle at the PSs. The relationship between EFs and PFs particles has been decuced from the appearance of particles arrays in barley thylakoids (35). Light-harvesting complex particles may make contact at the PSs, but are not shown as spanning the membrane until more data have been obtained. It is possible that the appearance of the light-harvesting complex particles at the ESs corresponds to the etched surface seen in Figure 29. It is assumed that the particles in light-harvesting complex vesicles correspond ot the particles missing from the PFs face of the chlorina-f2 mutant.

rium, which lacks both chlorophyll $b$ and phycobilisomes (11). In this mutant (47), as in chlorina- 2 , there is also an increase in the density of EF particles compared with the corresponding wild-type, so the particles may be close enough for direct energy transfer.

Studies with the isolated light-harvesting chlorophyll $a / b$-protein $(10,41)$ and the chlorina-f2 mutant show that the light-harvesting chlorophyll $a / b$-protein is involved in membrane stacking, but is not essential for grana formation, since chlorina- $f 2$ thylakoids form grana. Grana are also present in mutants which are deficient in photosystem II activity and have a greatly reduced density of EFs particles, but a normal PFs face and large amounts of lightharvesting chlorophyll $a / b$-protein $(29,36,37)$. The formation of grana with few EFs particles is difficult to explain if the light-harvesting chlorophyll $a / b$-protein is located in EFs particles. The slightly smaller size (about $20 \AA$ ) of the chlorina-f2 particles, both at the ESs and EFs planes (Tables IV and VI) may be due to the absence of another chlorophyll $b$-containing protein, chlorophyll $a / b$-protein 1 (25), which may be involved in transferring excitons from the light-harvesting chlorophyll to the reaction centre of photosystem II. The particles remaining on the PFs face of chlorina- $f 2$ thylakoids may correspond to photosystem I units.

Although some models show the lightharvesting complex as spanning the membrane $(3,4,21)$, there is only evidence that it is exposed at the outer surface (ESs) $(9,41)$. In liposomes containing light-harvesting complex, holes between particles are seen on the surface (Figure 29), while the arrays on the PS of thylakoids (28, 35) may be revealing the surface appearance of the light-harvesting complex on the other side of the membrane. This is taken into consideration in Figure 34, although new data may prove that the light-harvesting complex does indeed span the membrane. The molecular composition of light-harvesting complex particles has not been 
determined. However, a correlation between the size of freeze-fracture particles in the purple membrane of Halobacterium halobium and the electron diffraction map of this membrane has shown that each particle of $120 \AA$ diameter consists of several polypeptides, with a total molecular weight of about 300,000 (14). It is not valid to make a direct comparison between this membrane and light-harvesting complex liposomes since sufficient data are not yet available, but light-harvesting complex particles may contain about eight molecules of the light-harvesting chlorophyll $a / b$-protein, each of which contains approximately 14 molecules of chlorophyll (9).

\section{ACKNOWLEDGEMENTS}

I am grateful to Professor Diter von WetTSTEIN and Dr. C. Gamini Kannangara for helpful suggestions with the manuscript, and to Dr. Søren Rasmussen for suggesting the use of the computer system applied in this and earlier papers. I wish to thank JøRgen PeETz for updating the computer programs, ANN-SoFI STEINHOLTZ for printing the micrographs and Nina Rasmussen for drawing the figures. Financial assistance was provided by the Commission of European Communities contract 029-76-ES DK solar energy program. I would like to thank Dr. B. L. MøLLER for supplying purified light-harvesting complex and Dr. $\mathrm{O}$. MACHOLD for polyacrylamide gel electrophoresis (25).

\section{REFERENCES}

1. ANDERSON, J, M.: The molecular organization of chloroplast thylakoids. Biochim. Biophys. Acta 416, 191-235 (1975)

2. Anderson, J. M. \& R. P. Levine: Membrane polypeptides of some higher plants. Biochim. Biophys. Acta 333, 378-387 (1974)

3. Armond, P. A., L. A. Staehelin \& C. J. ARNTZEN: Spatial relationship of photosystem I, photosystem II, and the light-harvesting complex in chloroplast membranes. J. Cell Biol. 73, 400418 (1977)

4. Arntzen, C. J.: Dynamic structural features of chloroplast lamellae. In: "Current Topics in Bioenergetics (Eds. D. R. SANadi \& L. P. Vernon). Academic Press, N.Y. Vol. VIII, $111-$ 160 (1978)

5. Botrdman, N. K., O. BJörkman, J. M. ANDERSON, D. J. GOODCHILD \& S. W. ThORNE:
Photosynthetic adaptation of higher plants to light intensity: relationship between chloroplast structure, composition of the photosystems and photosynthetic rates. Proc. Third Internat. Cong. Photosyn., Rehovot. Vol. III, 1809-1827 (1974)

6. Boardman, N. K. \& H. R. Highkin: Studies on a barley mutant lacking chlorophyll $b$ I. Photochemical activity of isolated chloroplasts. Biochim. Biophys. Acta 126, 189-199 (1966)

7. Branton, D.: Interpretation of freeze-etch results. Proc. Eighth Internat. Cong. Electron Microsc., Canberra. Vol. II, 28-29 (1974)

8. Branton, D., S. Bullivant, N. B. Gilula, M. J. Karnovsky, H. Moor, K. Mühlethaler, D. H. Northcote, L. Packer, B. Satir, P. Satir, V. Speth, L. A. Staeheun, R. L. Steere \& R. S. Weinstein: Freeze-etching nomenclature. Science 190, 54-56 (1975)

9. Burke, J. J., C. L. Ditto \& C. J. Arntzen: Involvement of the light-harvesting complex in cation regulation of excitation energy distribution in chloroplasts. Arch. Biochem. Biophys. $187,252-263(1978)$

10. Burke, J. J., K. E. Steinback \& C. J. Arntzen: Analysis of the light-harvesting pigment-protein complex of wild type and a chlorophyll- $b$-less mutant of barley. Plant Physiol. 63, 237-243 (1979)

11. Diner, B. A. \& F.-A. Wollman: Functional comparison of the photosystem II center-antenna complex of a phycocyanin-less mutant of Cyanidium caldarium with that of Chlorella pyrenoidosa. Plant Physiol. 63, 20-25 (1979)

12. Dubertret, G. \& M. Lefort-Tran: Structural and functional organization of chlorophyll in the developing thylakoids of Euglena gracilis. In: "Chloroplast Development« (Eds. G. AkoyunoGLou et al.) Elsevier/North Holland. pp. 419425 (1978)

13. Elgsaeter, A.: A new freeze-etch unit for freeze-etch rotary shadowing, low temperature freeze-fracturing and conventional freeze-etching. J. Microsc. 113, 83-94 (1978)

14. Fisher, K. A. \& W. Stoeckenius: Freezefractured purple membrane particles: protein content. Science 197, 72-74 (1977)

15. Gerritsen, W. J., A. J. Verkleju, R. F. A. ZwaAl \& L. L. M. van DeEnen: Freeze-fracture appearance and disposition of band 3 protein from the human erythrocyte membrane in lipid vesicles. Eur. J. Biochem. 85, 255-261 (1978)

16. Goodchild, D. J., H. R. Highkin \& N. K. BOARDMAN: The fine structure of chloroplasts in a barley mutant lacking chlorophyll $b$. Exp. Cell Res. 43, 684-688 (1966)

17. Henriques, F. \& R. B. Park: Further chemical 
and morphological characterization of chloroplast membranes from a chlorophyll $b$-less mutant of Hordeum vulgare. Plant Physiol. 55, 763-767 (1975)

18. Henriques, F. \& R. B. Park: Development of the photosynthetic unit in lettuce. Proc. Nat. Acad. Sci. USA 73, 4560-4564 (1976)

19. Joliot, P., P. BenNoun \& A. Jollot: New evidence supporting energy transfer between photosynthetic units. Biochim. Biophys. Acta 305, 317-328 (1973)

20. Kannangara, C. G., S. P. Gough, B. Hansen, J. N. Rasmussen \& D. J. Simpson: A homogeniser with replaceable razor-blades for bulk isolation of active barley plastids. Carlsberg Res. Commun. 42, 431-439 (1977)

21. KirK, J. T. O. \& R. A. E. Tilney-Bassett: The Plastids. Elsevier/North Holland, Amsterdam. pp. 155-189 (1979)

22. Kübler, O., H. Gross \& H. Moor: Complementary structures of membrane fracture faces obtained by ultrahigh vacuum freeze-fracturing at $-196{ }^{\circ} \mathrm{C}$ and digital image processing. Ultramicroscopy 3, 161-168 (1978)

23. Lieberman, J. R., S. Bose \& C. J. Arntzen: Requirement of the light-harvesting complex for magnesium ion regulation of excitation energy distribution in chloroplasts. Biochim. Biophys. Acta 502, 417-429 (1978)

24. Machold, O., A. Meister, H. Sagromsky, G. Høyer-Hansen \& D. von Wettstein: Composition of photosynthetic membranes of wild-type barley and chlorophyll $b$-less mutants. Photosynthetica 11, 200-206 (1977)

25. Machold, O., D. J. Simpson \& B. Lindberg MøLler: Chlorophyll-proteins of thylakoids from wild-type and mutants of barley (Hordeum vulgare L.). Carlsberg Res. Commun. 44, 235254 (1979)

26. Margaritis, L. H., A. Elgsaeter \& D. BranTON: Rotary replication for freeze-etching. J. Cell Biol. 72, 47-56 (1977)

27. McDonnel, A. \& L. A. Staehelin: Biochemical and structural characterization of the lightharvesting chlorophyll $a / b$ protein complex from the chloroplast of higher plants. J. Cell Biol. 79, 318a (1978)

28. Miller, K. R.: A particle spanning the photosynthetic membrane. J. Ultrastruct. Res. 54, 159-167 (1976)

29. Miller, K. R. \& R. A. Cushman: A chloroplast membrane lacking photosystem II. Thylakoid stacking in the absence of the photosystem II particle. Biochim. Biophys. Acta 546, 481-497 (1979)

30. Miller, K. R., G. J. Miller \& K. R. MCINTyre: the light-harvesting chlorophyll-protein complex of photosystem II. J. Cell Biol. 71, 624-638 (1976)

31. Paradies, H, H., J. ZimmermanN \& U. D. Schmidt: The conformation of chloroplast coupling factor 1 from spinach in solution. J. Biol. Chem. 253, 8972-8979 (1978)

32. Rash, J. E., D. S. Copio, M. E. Eldefrawi \& C. S. Hudson: Practical labelling techniques for freeze-fracture. J. Cell Biol. 75, 247a (1977)

33. Ruben, G. C. \& J. N. Tel.Ford: The platinumcarbon shadow width increase as a function of known particle size on non-planar surfaces. Annu. Proc. Electron Microsc. Soc. Am., 33rd pp. 282-283 (1975)

34. Simpson, D. J.: Freeze-fracture studies on barley plastid membranes I. Wild-type etioplast. Carlsberg Res. Commun. 43, 145-170 (1978)

35. Simpson, D. J.: Freeze-fracture studies on barley plastid membranes II. Wild-type chloroplast. Carlsberg Res. Commun. 43, 365-389 (1978)

36. Simpson, D. J., G. Høyer-Hansen, N.-H. Chua \& D. vON WETTSTEIN: The use of gene mutants in barley to correlate thylakoid polypeptide composition with the structure of the photosynthetic membrane. Proc. Fourth Internat. Photosyn. Cong., Reading pp. 537-548 (1977)

37. Simpson, D. J., B. Lindberg Møller \& G. Hoyer-Hansen: Freeze-fracture structure and polypeptide composition of thylakoids of wildtype and mutant barley plastids. In: „Chloroplast Development« (Eds. G. Akoyunoglou et al.) Elsevier/North Holland. pp. 507-512 (1978)

38. Staemelin, L. A.: Chloroplast membrane structure. Intramembranous particles of different size make contact in stacked membrane regions. Biochim. Biophys. Acta 408, 1-11 (1975)

39. Staehelin, L. A.: Reversible particle movements associated with unstacking and restacking of chloroplast membranes in vitro. J. Cell Biol. 71. 136-158 (1976)

40. Staehelin, L. A., P. A. Armond \& K. R. Miller: Chloroplast membrane organization at the supramolecular level and its functional implications. Brookhaven Symp. Biology 28. 278-315 (1976)

41. Steinback, K. E., J. J. Burke, J. E. Mullet \& C. J. ARNTZEN: The role of the light-harvesting complex in cation-mediated grana formation. In: "Chloroplast Development« (Eds. G. AkoyunoGLOU et al.). Elsevier/North Holland. pp. 389400 (1978)

42. Thornber, J. P., R. S. Alberte, F. A. Hunter, J. A. Shiozawa \& K.-S. Kan: The organization of chlorophyll in the plant photosynthetic unit. Brookhaven Symp. Biology 28, 132-148 (1976) 
43. Weinstein, R. S., J. K. Khodadad \& T. L. SteCK: Fine structure of the band 3 protein in human red blood cell membranes: freeze-fracture studies. J. Supramolec. Struct. 8, 325-335 (1978)

44. Wettstein, D. von \& K. Kristiansen: Stock list for nuclear gene mutants affecting the chloroplast. Barley Genet. Newslett. 3, 113-117 (1973)

45. Wettstein, D. von, B. Lindaerg Møller, G. Høyer-Hansen \& D. J. Simpson: Genetic manipulation of photosystem I and II in chloroplast membranes. EC Solar Energy R\&D Programme. 3rd Co-ordination Meeting of Contractors, June 18-20, Brussels (1979)

46. Wiluson, J. H. M. \& M. R. Daver: Fraction 1 protein crystals in chloroplasts of isolated tobacco leaf protoplasts: a thin-section and freeze-etch morphological study. J. Ultrastruct. Res. 55, 303-311 (1976)

47. Wollman, F.-A.: Ultrastructural comparison of Cyanidium caldarium wild-type and III-C mutant lacking phycobilisomes. Plant Physiol. 63, 375-381 (1979) 